Florida International University FIU Digital Commons

$11-4-2016$

\title{
Targeted and Controlled Anticancer Drug Delivery and Release with Magnetoelectric Nanoparticles
}

Alexandra Rodzinski

Florida International University, arodz002@fiu.edu

DOI: $10.25148 /$ etd.FIDC001249

Follow this and additional works at: https://digitalcommons.fiu.edu/etd

Part of the Cancer Biology Commons, Medical Biophysics Commons, Medical Biotechnology Commons, Nanomedicine Commons, and the Oncology Commons

\section{Recommended Citation}

Rodzinski, Alexandra, "Targeted and Controlled Anticancer Drug Delivery and Release with Magnetoelectric Nanoparticles" (2016). FIU Electronic Theses and Dissertations. 2976.

https://digitalcommons.fiu.edu/etd/2976 


\title{
FLORIDA INTERNATIONAL UNIVERSITY
}

Miami, Florida

\section{TARGETED AND CONTROLLED ANTICANCER DRUG DELIVERY AND RELEASE WITH MAGNETOELECTRIC NANOPARTICLES}

\author{
A dissertation submitted in partial fulfillment of \\ the requirements for the degree of \\ DOCTOR OF PHILOSOPHY \\ in \\ BIOMEDICAL SCIENCES \\ by
}

Alexandra Rodzinski

2016 
To: Dean John Rock

College of Medicine

This dissertation, written by Alexandra Rodzinski, and entitled Targeted and Controlled Anticancer Drug Delivery and Release with Magnetoelectric Nanoparticles, having been approved in respect to style and intellectual content, is referred to you for judgment.

We have read this dissertation and recommend that it be approved.

Georg Petroianu

$\begin{array}{r}\hline \text { Stavros V. Georgakopoulos } \\ \hline \text { Osama A. Mohammed } \\ \hline \text { Carolyn D. Runowicz } \\ \hline \text { Sakhrat Khizroev, Major Professor }\end{array}$

Date of Defense: November 4, 2016

The dissertation of Alexandra Rodzinski is approved.

Dean John Rock College of Medicine

Andrés G. Gil

Vice President for Research and Economic Development and Dean of the University Graduate School

Florida International University, 2016 
(C) Copyright 2016 by Alexandra Rodzinski

All rights reserved. 


\section{DEDICATION}

All my work is dedicated to my wonderful family. 


\section{ACKNOWLEDGMENTS}

I would like to extend the strongest gratitude possible to my Professor, Dr. Sakhrat Khizroev, for being a truly remarkable mentor and for taking me into this uncharted territory of amazing nanotechnology. In addition to being absolutely brilliant, Professor Khizroev has this unique ability to leave you feeling uplifted and excited about the project after every conversation. I could not have asked for a better mentor and a better role model.

I am also very grateful to Dr. Rakesh Guduru for his kindness, company, and mentorship. I really wish we had longer to work together because he made every day in the lab a positive learning experience and something I would look forward to.

Additionally, I would like to thank my program directors, Dr. Alexander Agoulnik and Dr. Jeremy Chambers, for their extensive support and assistance. I would like to thank Dr. Irina Agoulnik for all her help throughout my entire PhD student career. I would also like to thank my committee for being nothing but supportive.

Finally, I would like to acknowledge everyone else who has helped me along the way. Whether it was teaching me something, giving me advice, or just being there for me, I am grateful. 


\title{
ABSTRACT OF THE DISSERTATION \\ TARGETED AND CONTROLLED ANTICANCER DRUG DELIVERY AND RELEASE WITH MAGNETOELECTRIC NANOPARTICLES \\ by
}

\author{
Alexandra Rodzinski \\ Florida International University, 2016 \\ Miami, Florida \\ Professor Sakhrat Khizroev, Major Professor
}

A major challenge of cancer treatment is successful discrimination of cancer cells from healthy cells. Nanotechnology offers multiple venues for efficient cancer targeting. Magnetoelectric nanoparticles (MENs) are a novel, multifaceted, physics-based cancer treatment platform that enables high specificity cancer targeting and externally controlled loaded drug release. The unique magnetoelectric coupling of MENs allows them to convert externally applied magnetic fields into intrinsic electric signals, which allows MENs to both be drawn magnetically towards the cancer site and to electrically interface with cancer cells. Once internalized, the MEN payload release can be externally triggered with a magnetic field. MENs uniquely allow for discrete manipulation of the drug delivery and drug release mechanisms to allow an unprecedented level of control in cancer targeting. In this study, we demonstrate the physics behind the MEN drug delivery platform, test the MEN drug delivery platform for the first time in a humanized mouse model of cancer, and characterize the biodistribution and clearance of MENs. We found 
that MENs were able to fully cure the model cancer, which in this case was human ovarian carcinoma treated with paclitaxel. When compared to conventional magnetic nanoparticles and FDA approved organic PLGA nanoparticles, MENs are the highest performing treatment, even in the absence of peripheral active targeting molecules. We also mapped the movement through peripheral organs and established clearance trends of the MENs. The MENs cancer treatment platform has immense potential for future medicine, as it is generalizable, personalizable, and readily traceable in the context of treating essentially any type of cancer. 


\section{TABLE OF CONTENTS}

CHAPTER

PAGE

CHAPTER 1: INTRODUCTION ………………………....................................... 1

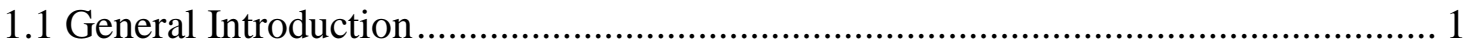

1.2 Study Motivation ........................................................................................ 2

CHAPTER 2: INTRODUCTION TO CANCER \& NANOMEDICINE ......................... 4

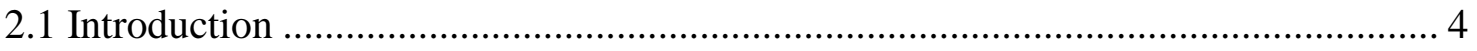

2.2 Cancer Physiology and Clinical Treatment Options ........................................ 4

2.2.1 Current Cancer Treatments ............................................................................. 4

2.2.2 Active \& Passive Drug Delivery .................................................................... 7

2.3 Organic Nanoparticles: Drug Delivery Platforms .......................................... 9

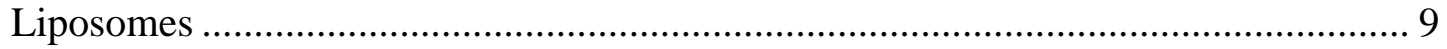

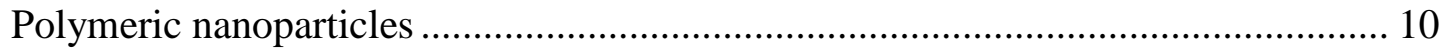

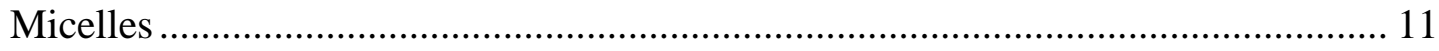

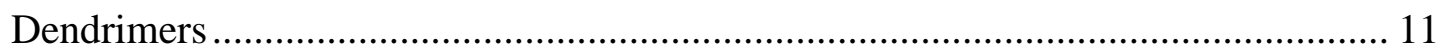

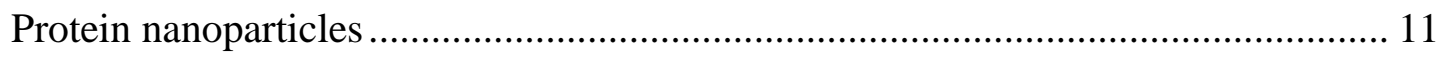

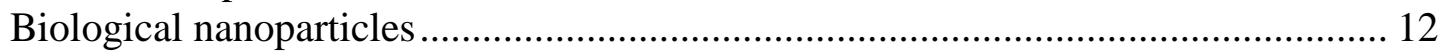

2.4 Inorganic Nanoparticles: Drug Carriers, Imaging Tools, and Treatment Agents ... 12

2.4.1 Metal nanoparticles (Weakly or Non -Magnetic)...................................... 13

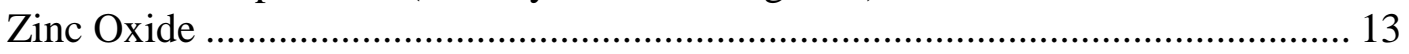

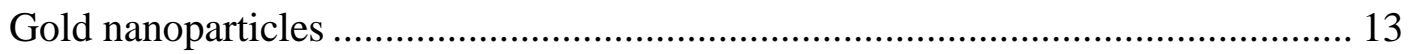

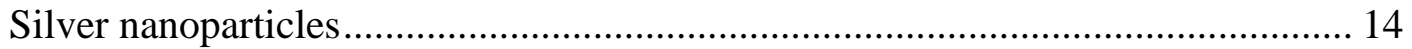

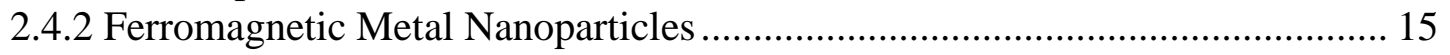

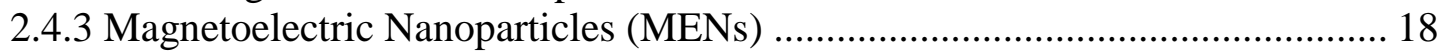

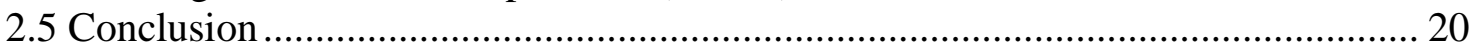

CHAPTER 3: INTRODUCTION TO MENS DRUG DELIVERY \& RELEASE.......... 22

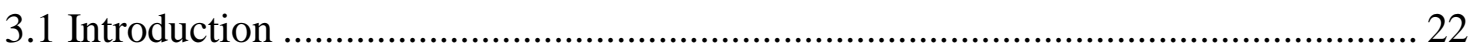

3.2 MEN HIV Drug Delivery \& Release ............................................................... 22

3.3 MEN Ovarian Cancer Drug Delivery \& Release .............................................. 28

3.4. MENs Drug Delivery \& Release Optimization ............................................. 34

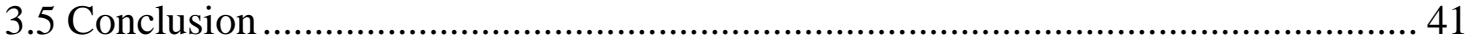

CHAPTER 4:

MENS USE A PHYSICAL MECHANISM TO ENTER CANCER CELLS ................. 42

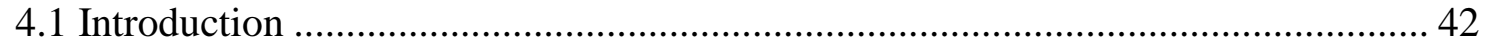

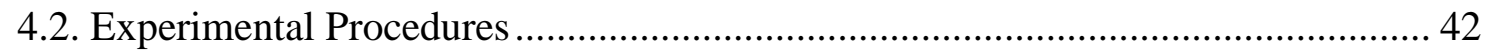

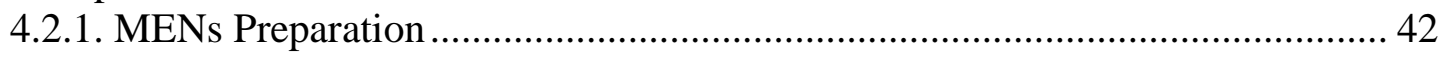

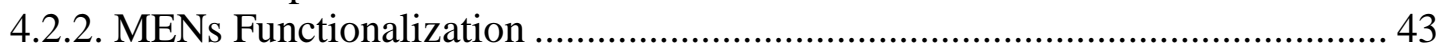

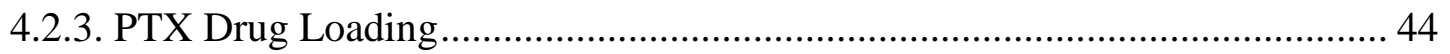

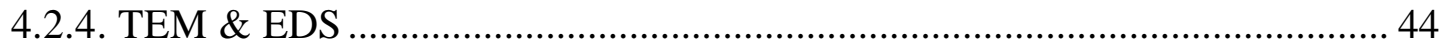


4.2.5. Magnetic Field Dependent Zeta Potential ..................................................... 44

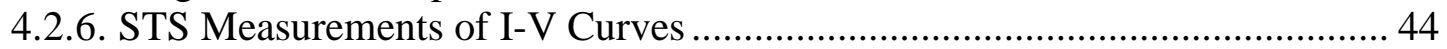

4.2.7. Vibrating Sample Magnetometry................................................................ 45

4.2.8. Fourier Transform Infrared Spectra ............................................................. 45

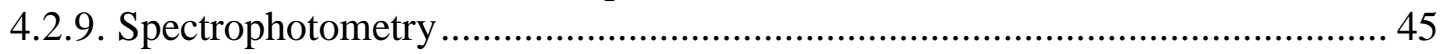

4.2.10. Cell Culture \& Lysis .............................................................................. 45

4.2.11. Scanning Probe Microscopy …………………......................................... 47

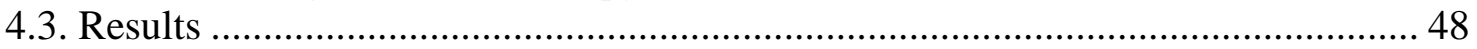

4.3.1 The Characterization of the Physical Features of MENs .................................. 48

4.3.2. The Cellular Internalization of MENs \& Their Payload ................................. 50

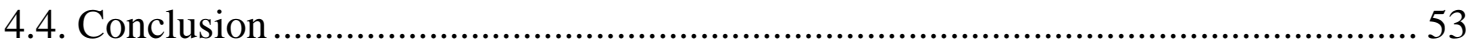

CHAPTER 5: MENS EFFECTIVELY CURE CANCER IN A MOUSE MODEL ........ 56

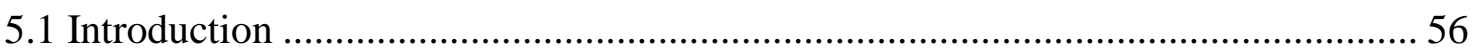

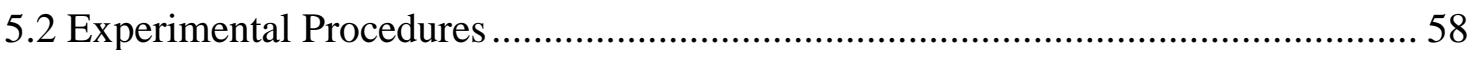

5.2.1. MEN \& MN Preparation and Drug Loading ………..................................... 58

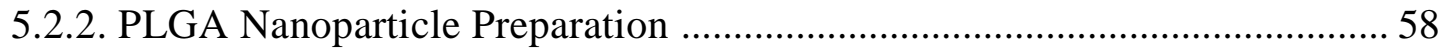

5.2.3. Antibody \& Ligand Tagging....................................................................... 59

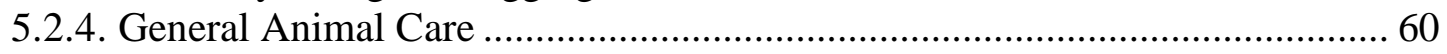

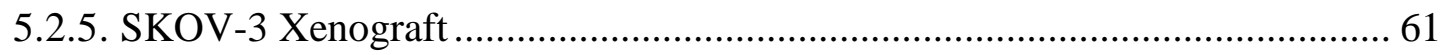

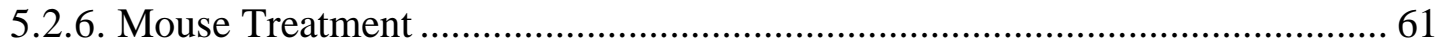

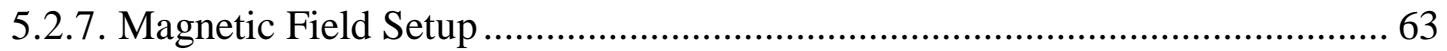

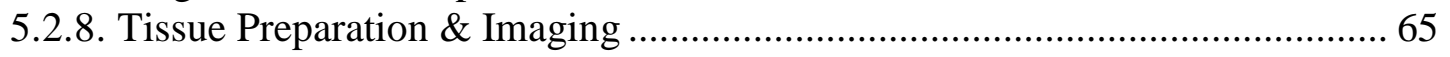

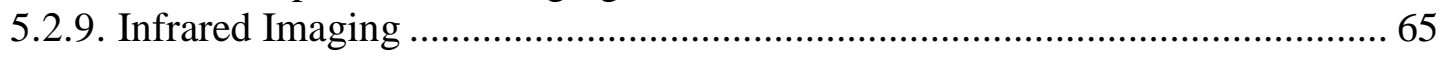

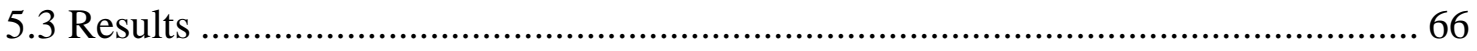

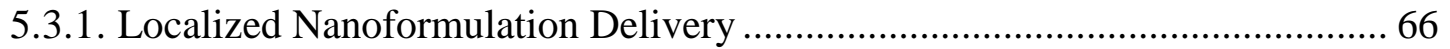

5.3.2. Comparisons of Nanoparticle Delivery Systems .............................................. 69

5.3.3. Size and A.C. Field Optimizations of the MENs Treatment ............................ 71

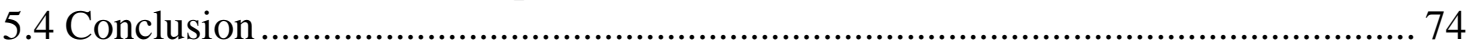

CHAPTER 6: BIODISTRIBUTON AND CLEARANCE OF MENS ............................. 80

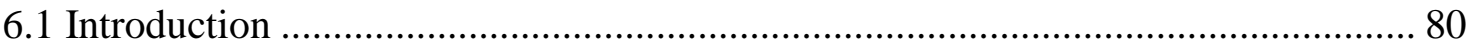

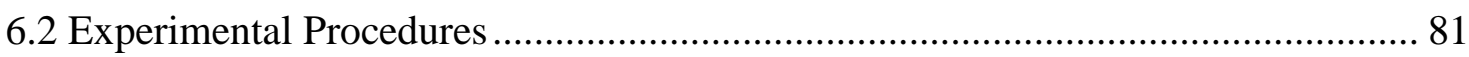

6.2.1. MENs Preparation \& Functionalization ........................................................... 81

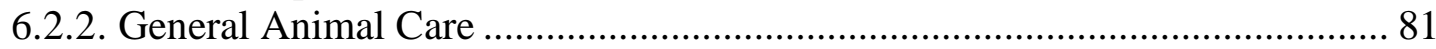

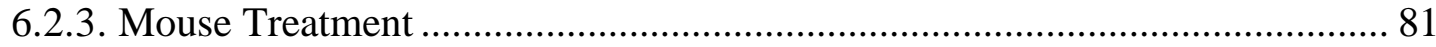

6.2.4. Tissue Collection \& Preparation ................................................................. 82

6.2.5. SEM Imaging with EDS Analysis ............................................................ 82

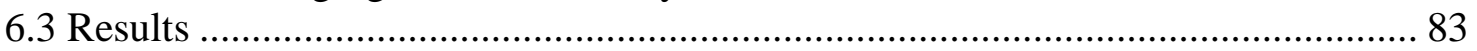

6.3.1. MEN Biodistribution and Clearance in Mice Treated for Cancer ................... 83

6.3.2. MEN Biodistribution and Clearance over Time .............................................. 91

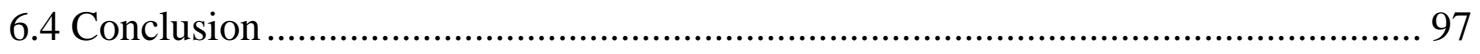




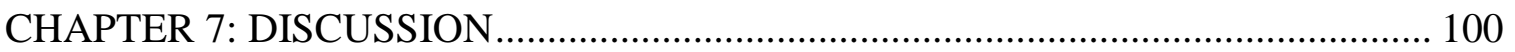

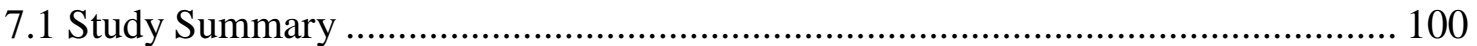

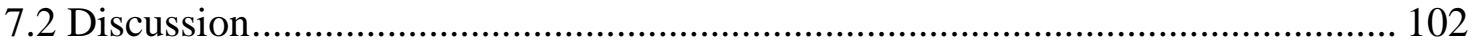

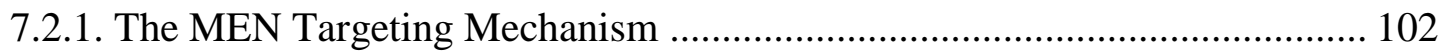

7.2.2. The MEN Payload Release Mechanism....................................................... 106

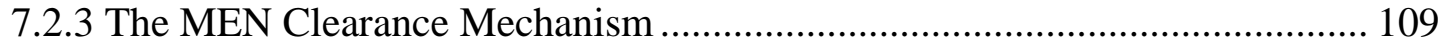

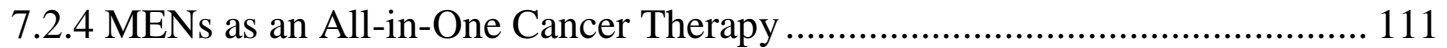

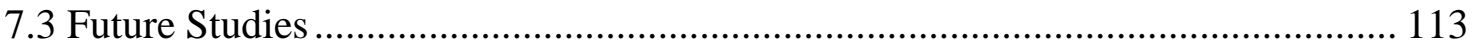

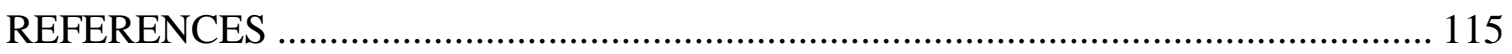

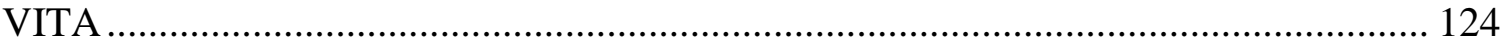




\section{LIST OF TABLES}

TABLE

PAGE

Table 1. NCI classifications of cancer treatments. .................................................... 5

Table 2. Preceding publications on MEN drug delivery.............................................. 22

Table 3. Quantification of MENs drug release in a magnetic field over time................ 26

Table 4. Quantification of GMO-MENs drug release in a magnetic field over time. ...... 31

Table 5. Trypan-Blue cell viability assay for nanoformulations and controls................. 34

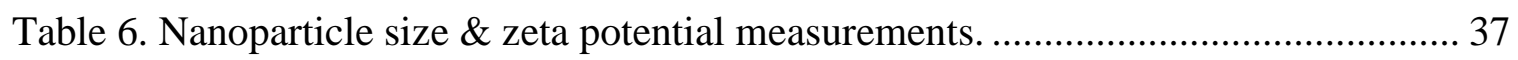

Table 7. Intact nanoformulation in peristaltic pump human circulation model

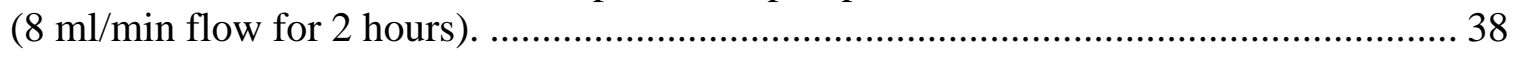

Table 8. MEN clusters in brain regions over time. ................................................. 97

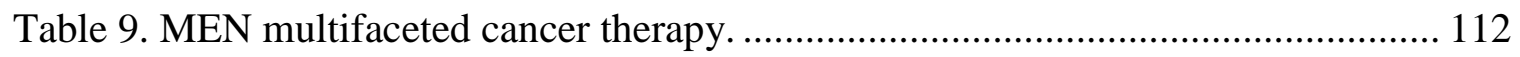




\section{LIST OF FIGURES}

FIGURE

PAGE

Figure 1. Magnetoelectric Nanoparticle Schematic................................................ 19

Figure 2. Illustration of the underlying physics of the a.c.-field-triggered release........... 23

Figure 3. Field strength and frequency dependence of the drug release........................ 24

Figure 4. Magnetic field strength and frequency over time determines payload release . 25

Figure 5. AFM of MENs \& Payload Stages ........................................................... 27

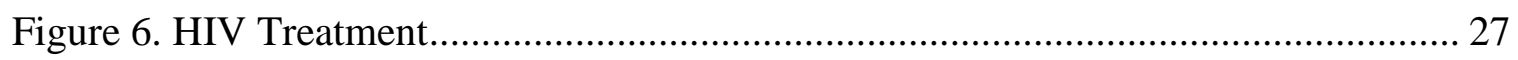

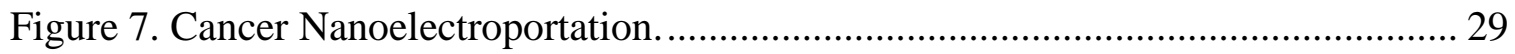

Figure 8. Free drug vs MN vs MEN formulation uptake by cancer cells...................... 30

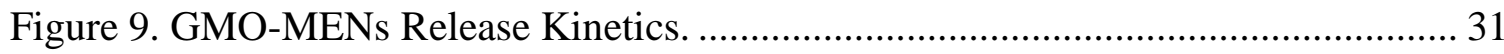

Figure 10. Field dependence of the drug uptake by SKOV-3 and HOMEC cells........... 32

Figure 11. Confocal imaging of SKOV-3 cell viability after treatment by different drug-carrier combinations with and without field. ....................................................... 33

Figure 12. SEM images of MENs with functionalized coatings ................................. 36

Figure 13. FTIR spectra of MENs with functionalized coatings................................ 37

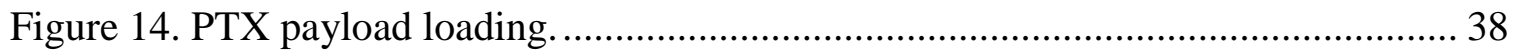

Figure 15. Magnetic field and frequency dependent drug release kinetics from

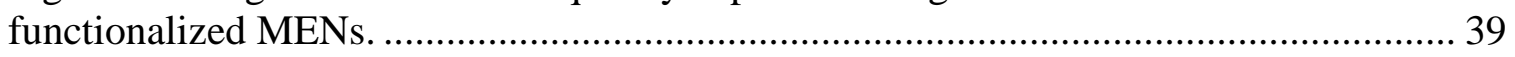

Figure 16. Functionalized MEN XTT cytotoxicity assay........................................ 40

Figure 17. Cell culture with magnetic field setup............................................... 47

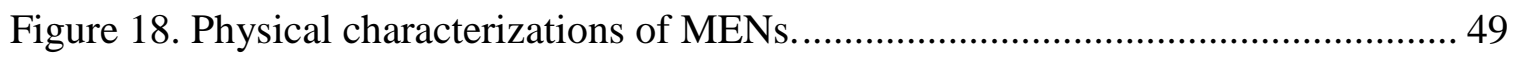

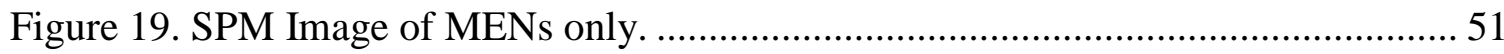

Figure 20. PTX calibration for spectrophotometer .............................................. 51 
Figure 21. SPM showing MENs only in field exposed cells.................................... 52

Figure 22. Field dependence of intracellular contents of treated cells. .......................... 53

Figure 23. PLGA nanoparticle imaged with AFM. ................................................. 59

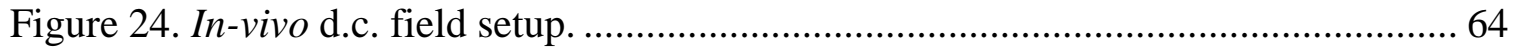

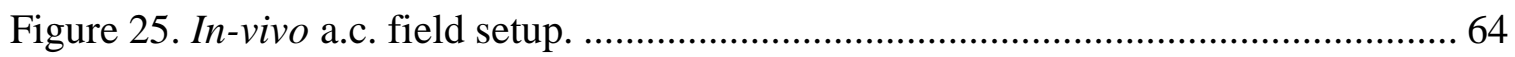

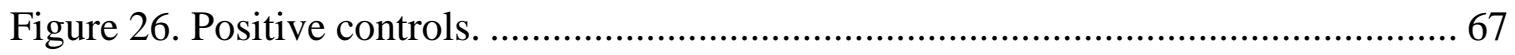

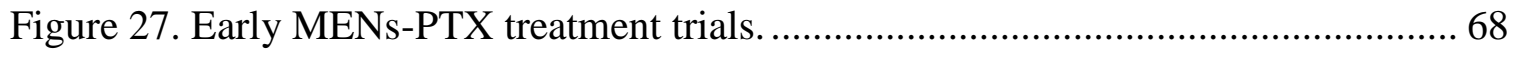

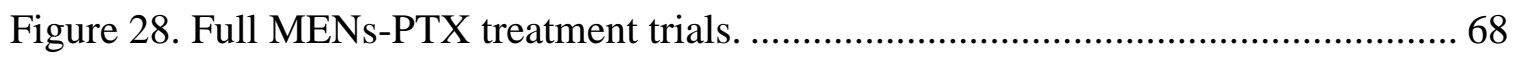

Figure 29. Efficacy of different nanocarrier platforms delivered via IV on tumor

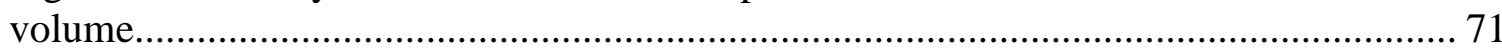

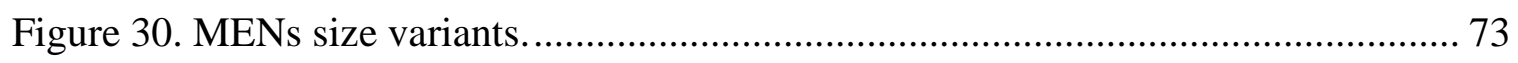

Figure 31. A.C. field application to different sized MENs........................................ 74

Figure 32. A fully cured mouse's progress...................................................... 76

Figure 33. HER2Sense monitoring of live animals. ............................................ 76

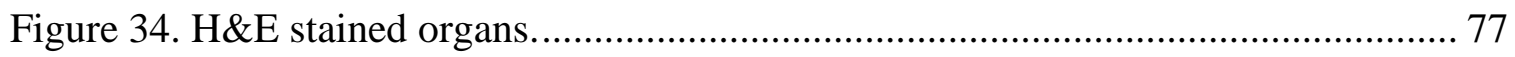

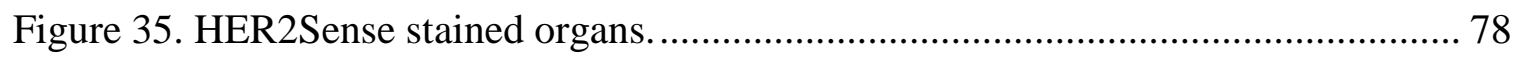

Figure 36. EDS analysis for MENs presence in tumor tissue .................................... 85

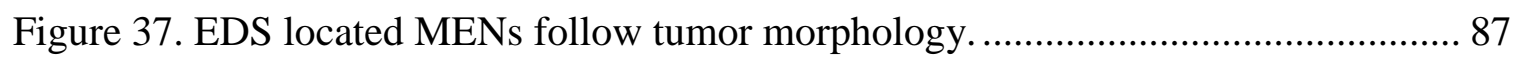

Figure 38. Subcutaneous vs IV localization of MENs.......................................... 88

Figure 39. MEN distribution in cancer treated mice ............................................... 90

Figure 40. Overall organ vs tumor MEN load in MEN treated mice. ........................... 90

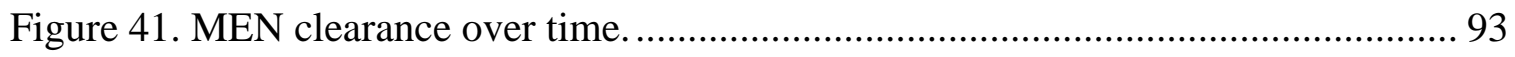

Figure 42. MEN organ distribution over time. ................................................. 95

Figure 43. $10 \mathrm{~nm}$ MEN cluster in glomerular region of kidney. ................................. 95 
Figure 44. Brain atlas showing located MENs.

Figure 45. MEN cancer targeting in circulation.

105

Figure 46. Magnetic fields control MEN cellular entry and drug release. 108

Figure 47. MENs generate no heat with applied fields...... 108 


\section{ABBREVIATIONS AND ACRONYMS}

MEN(s): Magneto-Electric Nanoparticle(s)

NIH: National Institute of Health

NCI: National Cancer Institute

EPR: Enhanced Permeability and Retention

PEG: Polyethylene Glycol

PLGA: Poly(D,L-Lactic-co-Glycolic Acid

MRI: Magnetic Resonance Imaging

SPION(s): Super Paramagnetic Iron Oxide Nanoparticle(s)

HIV: Human Immunodeficiency Virus

AZTTP: 3'-Azido-2',3'-Dideoxythymidine-5'-Triphosphate

A.C.: Alternating Current

D.C.: Direct Current

Oe: Oersted

BBB: Blood Brain Barrier

PBMC: Peripheral Blood Mononuclear Cell

MN(s): Magnetic Nanoparticle(s) (conventional)

GMO: Glycerol Mono-Oleate

SKOV-3: Human Ovarian Adenocarcinoma Cells

HOMEC: Human Ovarian Microvascular Endothelial Cells

EDC: Ethyl-3-(3-Dimethylaminopropyl) Carbodiimide

XTT: 2,3-bis-(2-methoxy-4-nitro-5-sulfophenyl)-2H-tetrazolium-5-carboxanilide 
PBS: Phosphate-Buffered Saline

DMSO: Dimethyl Sulfoxide

TEM: Transmission Electron Microscopy

EDS: Energy Dispersive Spectroscopy

STS: Scanning Tunneling Spectroscopy

I-V (curve): Current-Voltage (curve)

VSM: Vibrating Sample Magnetometer

FTIR: Fourier Transform Infra-Red

STM: Scanning Tunneling Microscopy

SPM: Scanning Probe Microscopy

AFM: Atomic Force Microscopy

MFM: Magnetic Force Microscopy

FN(s): Ferromagnetic Nanoparticle core(s)

NHS: N-hydroxysuccinimide

IV (injection): Intra-Venous (injection)

IR: Infra-Red

H\&E: Hematoxylin \& Eosin (stain)

Ba: Barium

Ti: Titanium

Si: Silicon 


\section{CHAPTER 1: INTRODUCTION}

\section{$\underline{1.1 \text { General Introduction }}$}

Cancer is one of leading causes of death worldwide, with 8.2 million deaths and 14 million new cases reported in 2012, and an expected number of 22 million new cancer cases within the next two decades [1]. In the United States, over a third of the population is expected to be diagnosed with cancer in their lifetime and in 2016 an estimated 595,690 people are predicted to die a cancer-related death while an estimated 1,685,210 new cases are predicted to arise [2]. Although cancer treatment options have both increased in quantity and improved in quality recently, there is still much work to be done to improve overall cancer survivability and quality of life while patients undergo cancer treatment. In fact, this year, the Obama Administration launched a billion dollar initiative called the National Cancer "Moonshot" initiative, which aims to greatly accelerate the speed and progress of cancer research to make more therapies available to more patients sooner [3]. This federal "Moonshot" directive highlights the urgency and priority that is currently placed on translatable cancer research.

Despite countless advances in cancer medicine, cancer treatment is still a huge challenge for researchers, health care providers, and patients alike. Unlike treating a microbial infection, where a discrete invader or unruly cohabitant is the source of illness, cancer poses the unique challenge of being almost indistinguishable from the rest of the organism. Cancer cells are a diseased version of the self, making isolating them for treatment extraordinarily difficult. Thus, cancer treatment depends on more subtle differences between the healthy cells and cancer cells (in comparison to the extensive 
differences between prokaryotic cells and eukaryotic cells in a bacterial infection, for example) for drug targeting.

While the cell biology of cancer does differ from that of healthy cells, it remains a challenge to effectively exploit these differences in practical applications regarding treatment and monitoring. Nanotechnologies offer a unique assortment of tools to help capitalize on some of the fundamental differences of cancerous tissues. A brief timeline

of the history of nanoparticles shows that nanoparticles have existed in nature long before humans, nanomaterials have been primitively utilized by humans for thousands of years with more advanced uses emerging hundreds of years ago, and the principles of nanomaterial properties have only been understood by humans for decades [4]. Modern day nanoparticles are reliably synthesized to come in a wide variety of formulations (and therefore properties) and are typically 1-100 $\mathrm{nm}$ in size, but can be anywhere between over a nanometer and under a micron. Nanoparticle treatments and treatment delivery systems are a very promising and exciting platform for cancer treatment. Nanoparticle based cancer treatment systems can complement, improve, or even entirely replace conventional cancer treatments.

\section{$\underline{1.2 \text { Study Motivation }}$}

Our group has pioneered the application of a new class of nanoparticles, magnetoelectric nanoparticles (MENs), to biomedical sciences. This unique inorganic nanoparticle, which exhibits strong magneto-electric coupling (discussed in Chapter 2), opens the door to a plethora of medical applications. We have already demonstrated the use of MENs as customizable drug-delivery platforms (discussed in Chapter 3) and deep- 
brain stimulation platforms [5], [6], and are currently working on other novel applications of this technology.

One of our primary goals is to develop a cancer therapy that can universally be used for the majority of cancer patients. The ideal cancer therapy would be customizable based on specific patient needs, but would rely on generally applicable properties of cancer anatomy and physiology to be relevant to all but the most unique cases. Such a treatment would have minimal side effects and would also, ideally, be readily imaged for monitoring the treatment. We believe that MENs have the potential to be the ideal cancer treatment because the MENs nanomedicine platform conforms to the ideals of being (a) generalizable to almost any cancer, (b) highly specific to cancer, thereby reducing side effects, and (c) readily imaged with current technology.

This dissertation focuses on the application of MENs as a highly targeted and controlled drug delivery and release platform for the treatment of cancer. We wanted to demonstrate three main concepts in the scope of this dissertation work:

1. Confirm that MENs are able to discriminately enter cancer cells (while avoiding healthy cells) by crossing the cell membrane to reach the cytoplasm with the payload intact when influenced by an externally applied magnetic field (Chapter 4).

2. Test the MEN cancer drug delivery platform for the first time in a mouse model to establish the successful translation of the treatment from in-vitro to in vivo (Chapter 5).

3. Establish the biodistribution and clearance patterns of MENs in the mouse model (Chapter 6). 


\section{CHAPTER 2: INTRODUCTION TO CANCER \& NANOMEDICINE}

\subsection{Introduction}

The scope of this literature review covers the relevant aspects of cancer that are commonly exploited by nanotechnology for cancer treatment and the nanotechnologies currently utilized for this purpose. Specifically, the formation of the enhanced permeability and retention effect will be discussed, as it is pertinent to all nanomedicines. Active targeting by means of surface molecules is also introduced. Then, both organic and inorganic nanotechnologies will be briefly introduced in the context of their drug delivery capabilities and cancer treatment potentials.

\section{$\underline{2.2 \text { Cancer Physiology and Clinical Treatment Options }}$}

\subsubsection{Current Cancer Treatments}

While the total incidence rates of cancer are still climbing, the treatment options and survival rates are growing as well. Indeed, cancer related death rates have been falling on average 1.5\% each year between 2004-2013 and the 5 year survival has increased to $68.9 \%$ in 2008 compared to $48.7 \%$ in 1975 [2], [7]. Cancer is no longer the death sentence it used to be, but is still associated with morbidity from cancer therapies. Table 1 summarizes the National Institute of Health (NIH): National Cancer Institute (NCI) classifications of the main categories of current clinical cancer treatments. 
Table 1. NCI classifications of cancer treatments.

\begin{tabular}{|c|c|c|}
\hline Treatment Type & Description & Main Limitations \\
\hline Surgery & $\begin{array}{l}\text { Tumor excision via manual } \\
\text { removal or tumor destruction } \\
\text { via freezing, heating, laser, or } \\
\text { photodynamic therapy. }\end{array}$ & $\begin{array}{l}\text { Only applicable to well } \\
\text { contained tumors at known and } \\
\text { accessible locations. }\end{array}$ \\
\hline $\begin{array}{l}\text { Radiation } \\
\text { Therapy }\end{array}$ & $\begin{array}{l}\text { External or internal application } \\
\text { of high doses of radiation to } \\
\text { attack cancer cells. }\end{array}$ & $\begin{array}{l}\text { Extensive healthy cell damage } \\
\text { related side effects. }\end{array}$ \\
\hline Chemotherapy & $\begin{array}{l}\text { Using pharmaceutical agents to } \\
\text { chemically attack cancer cells. }\end{array}$ & $\begin{array}{l}\text { Extensive healthy cell damage } \\
\text { related side effects. }\end{array}$ \\
\hline $\begin{array}{l}\text { Targeted } \\
\text { Therapy }\end{array}$ & $\begin{array}{l}\text { Exploits the unique cellular } \\
\text { properties of cancers to develop } \\
\text { very specific treatments. }\end{array}$ & $\begin{array}{l}\text { Challenging drug development } \\
\text { process due to extensive possible } \\
\text { targets; development of } \\
\text { treatment resistance; side effects } \\
\text { from healthy cell off-targeting. }\end{array}$ \\
\hline $\begin{array}{l}\text { Hormone } \\
\text { Therapy } \\
\text { (Targeted } \\
\text { Therapy Subtype) }\end{array}$ & $\begin{array}{l}\text { Modifying the patient's } \\
\text { hormones to reduce hormones } \\
\text { beneficial to cancer. }\end{array}$ & $\begin{array}{l}\text { Only applicable to cancers } \\
\text { strongly associated with } \\
\text { hormones; hormone imbalance } \\
\text { related side effects. }\end{array}$ \\
\hline $\begin{array}{l}\text { Immunotherapy } \\
\text { (Targeted } \\
\text { Therapy Subtype) }\end{array}$ & $\begin{array}{l}\text { Assisting the patient's own } \\
\text { immune system to attack cancer } \\
\text { cells using monoclonal } \\
\text { antibodies, cytokines, adoptive } \\
\text { T cell transfer, or other } \\
\text { biological methods. }\end{array}$ & $\begin{array}{l}\text { Use not as widespread as other } \\
\text { therapies; immune response } \\
\text { related side effects. }\end{array}$ \\
\hline
\end{tabular}

The numerous treatments available provide many options for cancer patients.

Surgery is a good course of action when possible; however, it is simply not an option for every cancer type and extent of progression. Chemotherapy [8], [9] and radiation therapy [10]-[12] can also be very effective treatments, but typically at the cost of extensive collateral damage to healthy cells that result in considerable side effects. Targeted therapy [13], therefore, provides an attractive option for treatment in that it leads to “precision medicine" by using targets unique to cancer [14]-[16]. It provides higher specificity to cancer cells to reduce side effects and can be tailored to potentially any 
cancer with enough research into cancer biomarkers or any other targetable components. This new level of specificity for cancer cells will improve upon the already established, albeit weaker, specificity of conventional treatments.

Considering that one of the most important defining parameters of cancer cells is their unrestricted growth and division, the processes of halting new DNA synthesis, destroying existing DNA, and interfering with mechanisms of mitosis are frequently investigated for anti-tumor potential [17]. There are numerous drug options for mitotic inhibitors (including: microtubule stabilizers, kinetochore, centromere, and centrosome interferes; other mitotic cytoplasm protein binders) each of which can lead to cell death or an alternative pathway that halts cellular division and progress [17]-[19]. While this type of drug is still cytotoxic to healthy cells, the uncontrolled division characteristic of cancer cells will make them more heavily affected than regular, slow-dividing cells. Normal rapidly dividing non-cancerous cells, like hair follicles, tend to also be noticeably affected in these treatments, resulting in the hair loss associated with undergoing cancer treatment.

Another distinctive feature of tumors is their recruitment of new blood vessels to maintain perfusion for the growing mass of cancer cells. Angiogenesis in general is directed by a local balance of promoters and inhibitors [20], [21]. In the case of many cancers (as well as healthy, normally angiogenic tissues like ovarian follicles), the net balance is heavier on angiogenic promoters than on inhibitors, thereby causing nearby blood vessels to sprout growth into the tissue. Tumors that fail to recruit new blood vessels are typically stunted in growth by the limiting factor of nutrient diffusion from nearby vessels. These under-perfused tumors exhibit necrosis in areas where growth has 
exceeded the influence of nutrient diffusion, and will maintain a relatively stable size as cells further away from the nutrient source die off. Likewise, tumors located sufficiently far from blood vessels are less capable of disseminating into circulation and tend to remain in a benign state. Thus, anti-angiogenic drugs are another good treatment option in that they hinder the growth potential of the tumor [22].

Compared to drugs targeted to unique functions of cancers like rapidly mitotic division or angiogensis, nanocarriers allow for even fewer unintended recipients of the drugs by establishing greater directing power to cancer cells and away from healthy cells (like the naturally rapidly dividing hair follicles or the periodically angiogenic ovarian follicles, for example). Nanocarriers also allow for a smaller drug dose to be used for the desired effect, as less of the drug is lost en route and, therefore, fewer side effects are incurred from having bioavailable drug in the physiological system. Furthermore, if a reliable drug binding is accomplished until the designated target is reached with high specificity, other generally cytotoxic agents can potentially be used to treat a variety of cancers. This would greatly increase the drug options beyond those that do not overwhelmingly affect healthy cells into potentially more potent cancer cell eliminators.

\subsubsection{Active \& Passive Drug Delivery}

The enhanced permeability and retention (EPR) effect is a unique characteristic of tumors that arises from the combination of malformed vascular and lymphatic vessels. This allows macromolecules to both enter and linger inside tumors, making it exploitable to improve treatment delivery [23]. The vasculature of tumors is unique in several ways [24]. First, these vessels lack the typical architecture of branching larger vessels into smaller ones with regular spacing, and are prone to collapse by crushing from 
neighboring proliferating tumor cells. This causes an irregular arterial to venous pressure difference which slows down blood flow in the vessels and lowers the perfusion of surrounding tissue. Secondly, the cellular composition of tumor vasculature is different in that there are wide inter-endothelial junctions along with a surplus of vesicular transendothelial channels and fenestrations. These pores in the vasculature can be up to several hundred nanometers wide, and contribute to the "leaky" property of tumor blood vessels. Tumors also lack an adequate lymphatic drainage system, forcing macromolecules to accumulate at the site of the perforation [25]. Proliferating cancer cells crush and collapse existing lymphatic vessels [23], and unlike the characteristic promotion of angiogenesis by cancer cells to maintain perfusion, lymphatic vessels are not sufficiently regenerated in cancerous tissue to maintain adequate lymphatic drainage.

The enhanced permeability of tumor vasculature is coupled with the retention caused by inadequate lymphatic drainage to cause the EPR effect, which can allow cancer therapies of the correct size and properties to accumulate inside tumors [26]. This type of drug delivery is considered passive, relying on inherent properties of the tumor macroenvironment. By comparison, active targeting relies on molecule-facilitated guidance to specific components unique to the surfaces of diseased cells. Immunological agents such as monoclonal antibodies [27] or other ligands are selected to have high specificity for cancer markers, and can actively seek out cancer cells for drug delivery [28]. Compared to EPR, active targeting allows for smaller cell masses to be targeted, but requires a more complex design to have a strong enough affinity for an antigen that is consistently present on cancer and absent on healthy cells. The heterogeneity of cancer cells between individuals and even within the same individual must be considered when selecting an 
active targeting molecule, making a fully comprehensive cancer treatment a challenge to develop using immunotherapy-assisted drug targeting exclusively [29].

Nanoparticles are very effective exploiters of the EPR mechanism and can be used to passively accumulate drugs inside tumors. Likewise, nanoparticles can be formulated to actively target cancer-specific molecules for drug delivery. Combining the passive and active targeting approaches can lead to even better localization of drugs into tumor cells [30].

\subsection{Organic Nanoparticles: Drug Delivery Platforms}

Organic nanoparticles can be synthetic or natural in origin. They have been accepted in biomedical applications for longer than inorganic particles and are favored with respect to having no biocompatibility or retention issues. However, the trade-off for these fully biocompatible properties is a very quick release of their contents due to the inherent capacity to break down in the biological system to which the particles are introduced. Even though there is minimal control of when organic nanoparticles decompose and trigger their payload release, they do offer a considerable improvement compared to utilizing free-drug.

Liposomes are one of the oldest nanoparticle drug carriers designed to improve pharmacokinetics and biodistribution [23]. Liposomes are composed of various lipids in a mono- or bi-layer forming a spherical vesicle surrounding an aqueous core. They can be designed to range greatly in size (from about $10 \mathrm{~nm}$ to over $1 \mu \mathrm{m}$ ) and in surface properties, like charge and functionality, using varied combinations of commercially available lipids [31]. Another benefit of the aqueous core surrounded by a layer of lipid 
structure is that both hydrophilic and hydrophobic drugs can be loaded into each compartment, respectively. However, liposomes suffer from a low encapsulation capacity, poor drug solubility, and instability in the bloodstream that leads to a very quick-burst release of loaded drugs. Some of these shortcomings can be addressed with functionalized coatings, like polyethylene glycol (PEG), which will increase the stability and circulation half-life of the liposomes [32]. However, even uncoated liposomes already offer an improvement over the half-life of free drug.

Polymeric nanoparticles are composed of various polymers such as poly (lactic acid), poly(D,L-lactic-co-glycolic acid) (PLGA), dextran, chitosan, etc [33]. These polymers are biocompatible and tend to be biodegradable into the monomer units, which can then be metabolized normally by the body. The degradation process will release the drugs encapsulated inside. The rate of degradation and, therefore, the drug kinetics are determined by the polymeric nanoparticle design aspects such as hydrophobicity, size, dispersity index, and crystallinity [31]. Polymeric nanoparticles tend to be relatively stable due to the hydrophilic shell providing steric protection to the nanoparticle while the hydrophobic core holds a high capacity of the drug to be delivered [23]. Another benefit is that a wide variety of drugs can be loaded inside polymeric nanoparticles, including small molecules of both hydrophobic or hydrophilic varieties and even macromolecules such as nucleic acids or proteins [34]. Polymeric nanoparticles can also be given a functionalized surface coating, such as PEG, to further modify their physiological properties. 
Micelles can also be a type of polymeric nanoparticles, however they can also be made entirely from lipids, similar to liposomes [35]. Micelles tend to range from 10-100 nm and are composed of a hydrophobic core surrounded by amphiphilic surfactant molecules [36]. The key difference between liposomes and lipid micelles is that micelles lack the aqueous core of liposomes and are instead designed specifically to hold hydrophobic drugs.

Dendrimers are branched macromolecules composed of monomers such as nucleotides, amino acids, or sugars from natural or synthetic sources [23]. Dendrimers can be designed to conjugate to drugs through hydrophobic interactions, hydrogen bonds, or chemical linkages within the cavities of the core created by the branching architecture. The surface can also contain functional groups for further modification. Dendrimers tend to be small $(5-10 \mathrm{~nm})$ and globular structures with notable monodispersity, where the drug release kinetics can be readily controlled through the cleavable chemistry in the polymer chains [31].

Protein nanoparticles are also an attractive drug delivery platform due to the extensive functionalities and the fully biodegradable and metabolizable nature of natural proteins. Protein nanoparticles can readily interact with both drug and solvent due to their amphiphilicity, and have modifiable surfaces onto which a wide variety of drugs and targeting molecules can be attached [37]. One of the most commonly studied protein nanoparticles is albumin (of both bovine and human serum), which is a natural soluble carrier of endogenous hydrophobic molecules. Other water soluble protein nanoparticles include gelatin, elastin, and soy proteins. Insoluable proteins are also available in the 
forms of gliadin, legumin, and zein. One of the major benefits of food-based protein nanoparticles, such as milk and whey derived nanoparticles, is that the materials are readily available and easy to formulate, and that there already exists extensive knowledge on the safety of their use.

Biological nanoparticles utilize existing uniceulluar organisms such as bacteria to serve as drug carriers [31]. A biological cell offers many options for loading drugs of various solubilities and charges, and an intact cell offers considerable stability in physiological conditions. While this delivery platform is liable to induce an immunological response when administered, the removal of the nucleus ensures no pathogenic activity of the drug-loaded cell.

\subsection{Inorganic Nanoparticles: Drug Carriers, Imaging Tools, and Treatment Agents}

Inorganic nanoparticles are mostly composed of an inert metal substance that is biocompatible, with all other properties being dependent on the exact metals in the formulation. Arguably, the most significant benefit of metal nanoparticles is the tripletreatment potential: imaging, drug delivery, and inherent cancer cell cytotoxicity. Similar to their organic nanoparticle counterparts, inorganic nanoparticles can be drug loaded and functionally coated for passive and active drug delivery. However, they have the added benefit of being readily traceable through medical imaging technology and of being uniquely cytotoxic to cancer cells with or without extrinsic stimulation. The generation of heat or reactive oxygen species, for instance, places metal nanoparticles into a distinctive treatment class that can further be augmented with the same drug delivery aspects as other nanoparticles types. 


\subsubsection{Metal nanoparticles (Weakly or Non-Magnetic)}

In this section, metal nanoparticles refer to particles composed of weakly or nonmagnetic metals that are used for cancer treatment. These nanoparticles are usually formulated from the frequently studied noble metals (i.e. gold and silver) or zinc based compounds, but can also be made of numerous other, generally non-magnetic metals, including, but not limited to, silicon, cerium, barium, bismuth, calcium, copper, magnesium, and titanium [38], [39]. The following section is a summary of some of the functional highlights of zinc, gold, and silver nanoparticles in regards to cancer treatment selected from the extensive and well-written Sharma et al. review [38].

Zinc Oxide nanoparticles can be imaged based on their inherent photoluminescence. These particles are also strong reactive oxygen species generators due to the wide band-gap semiconductor nature of the crystalline structure, which allows electrons to travel between gaps and create unoccupied states $(\mathrm{H}+)$. Both the electrons and the electron holes diffuse to the particle surface where superoxide and hydroxyl radicals are created upon the reaction of oxygen with electrons, and hydroxyl ions with the electron holes, respectively. The generation of reactive oxygen species is further instigated by the application of ultraviolet light, making for an even more potent cytotoxicity at a targeted site.

Gold nanoparticles can readily be followed in a biological system using multiple imaging platforms including optical imaging, magnetic resonance imaging (MRI), computed tomography, photoacoustic imaging, and flow cytometry. They are also valuable in photothermal therapy, where an extrinsic application of energy causes localized heating through the nanoparticles. This is 
because gold nanoparticles are an example of a plasma metal, where the nanoparticle system has an equal number of fixed positive ions and mobile conduction electrons, which will result in an oscillating electron charge in resonance with visible light frequency when irradiated with an electromagnetic wave. The size of the gold nanoparticle determines the extent of the energy loss in the form of scattering and absorption. Larger particles have a higher scattering efficiency that makes them superior for imaging, while smaller particles are better absorbers and are therefore preferred for their effective conversion of energy into heat in photothermal therapy. In addition to effective photothermal conversion for hyperthermic destruction, gold nanoparticles are also radiosensitizers, allowing radiation therapy to work more effectively on cancer cells.

Silver nanoparticles have similar plasmonic features to gold, making them comparably effective at absorbing and scattering applied light for imaging and photothermal therapy, respectively. However, silver has a higher plasmon excitation efficiency and has a plasmon resonance that can be tuned to any visible light wavelength. Another unique feature of silver nanoparticles is their neutralization of glutathione and thioredoxin, which are the primary reactive oxygen species neutralizing proteins. This destruction of antioxidant defenses will allow an accumulation of reactive oxygen species that can ultimately cause enough damage to induce cell death.

Finally, mesoporous silica nanoparticles are also an inorganic nanoparticle class which offers unique utilities. Characterized by their highly customizable pores, mesoporous silica nanoparticles offer extensive surface area and volume within the pores 
for stable, high encapsulation capacity drug loading while maintaining biocompatibility and controlled release mechanisms [40]. The dissolution of the encapsulated drugs from mesoporous silica nanoparticles is preferable over the organic nanoparticle counterparts' in that the payload is maintained in amorphous or noncrystaline states in the chemically stable and inert nanocarrier [41]. Furthermore, mesoporous silica nanoparticles can be tagged with fluorescent agents for fluorescent bioimaging or formulated to incorporate MRI contrast agent nanoparticles, without or without further ligand targeting to specific tissue sites, for MRI imaging.

\subsubsection{Ferromagnetic Metal Nanoparticles}

Ferromagnetic nanoparticles are a subtype of nanoparticles that can be classified as having a significantly magnetic nature. These particles are typically made of iron, but can also be fully or partially composed of nickel or cobalt. Like other metal nanoparticles, ferromagnetic particles can be (a) functionalized for molecular targeting, (b) drug-loaded, and (c) drug-free tumor treatments in the form of hyperthermia or reactive oxygen species generation in response to non-toxic wavelength radiation. The unique benefit of magnetic metal based nanoparticles is the sensitivity to magnetic fields, which can be safely applied externally to induce the selective cytotoxic effects in addition to external directing of nanoparticles to target sites [42]. Likewise, a permanent magnet can be non-invasively implanted for a constant pull of magnetic nanoparticles to a more internally located tumor and can then be safely removed upon completion of the treatment. Other than active molecular targeting and administration in the vicinity of the tumor site when possible, there is little that can be done to help direct nanoparticle 
treatments to their intended destination post-administration. The EPR effect can be complemented with strategically placed magnetic fields, and magnetic field gradients can be used to create a customized attraction of magnetic nanoparticles to the intended treatment region [43].

Some of the most commonly studied ferromagnetic materials are iron oxides, especially magnetite $\left(\mathrm{Fe}_{2} \mathrm{O}_{4}\right)$, which occurs naturally in both prokaryotic and eukaryotic organisms for magnetic field sensing [44]. Magnetite has unique physical properties (i.e. magnetics based on a small size with large surface area) that make it well suited for biological applications [45], and it is readily synthesized to be biocompatible [46]. While there is some concern for ferromagnetic aggregation of magnetic nanoparticles, this can be addressed with careful magnetization of the particles. Unlike the constant magnetization of bulk iron, super paramagnetic iron oxide nanoparticles (SPIONs) are used precisely because they exhibit a magnetism of zero when not under the effect of a magnetic field [38], [47]. This allows for the magnetic properties of the particles to be activated only when needed (i.e. when at the tumor site instead of when traveling through the blood). When SPIONs are unaffected by a field, there are random fluctuations in the magnetization direction of each particle caused by the thermal energy overcoming the coupling forces between neighboring particles above the Curie temperature [43]. As each particle shifts its magnetic moment to align with an applied magnetic field, the overall magnetic moment of the particles collectively is no longer null and an overall polarization is established. The magnetization can then be returned to zero immediately by applying a field of equal strength in the negative direction, or it will return slowly if the magnetic field is simply removed, as a spontaneous random orientation of 
nanoparticle magnetic spins is achieved over time. This principal ensures that no agglomeration will occur before the particles localize at the site and after treatment is complete.

If alternating external magnetic fields are applied, heat is released as the particles lose energy from the quick reorientation of the magnetic dipoles proportional to the strength and frequency of the applied field. This makes magnetic nanoparticles well suited to magnetic field induced hyperthermia treatment [48], [49]. In fact, hematite $\left(\mathrm{Fe}_{2} \mathrm{O}_{3}\right)$ particles have been used to thermally treat metastasized cancers in the lymph nodes almost 60 years ago [50]. Furthermore, mild hyperthermia is synergistic with other treatment types, making a nanoparticle that induces hyperthermia and delivers a secondary treatment highly desirable [51]. While larger particle sizes result in higher saturation magnetization values and therefore stronger magnetic hyperthermia results, when magnetic nanoparticles exceed a certain size, they surpass the super-paramagnetic limit and become magnetic; for SPIONs, that limit was reported to be 30-40 nm [39], and most SPIONS have a core size under $10 \mathrm{~nm}$ to establish sufficient thermal energy to allow the magnetic direction fluctuation [47]. It is therefore important to balance the higher tumor accessibility of smaller particles against the stronger magnetization potential but also the stronger aggregation potential of larger particles [52].

Magnetic nanoparticles are excellent contrast agents for MRI as they create nonuniform magnetic fields under externally applied magnetic fields by helping protons in the surrounding environment relax at a faster rate [53]. The same type of functionalized coatings that assist in drug delivery can be utilized to assist in stronger localization for imaging [54]. More recently, a technique referred to as "magnetic nanoparticle imaging" 
emerged as a more sensitive version of MRI imaging [55], capable of real time high resolution 3D imaging [56]. This technique relies on the non-linear magnetization curve of ferromagnetic nanoparticles to reorient to a static selection field after an initial orientation by an applied oscillating drive field in a field-free target location.

\subsubsection{Magnetoelectric Nanoparticles (MENs)}

MENs are the latest development in magnetic nanoparticle technology, and include all the benefits of magnetic nanoparticles along with the benefits of the novel electric properties [57]. In addition to being able to respond to an applied magnetic field in all the same ways as conventional magnetic nanoparticles, MENs are also nanoconverters of magnetic fields to intrinsic electric signals due to their strong magnetoelectric coupling [58]. This non zero magneto-electric moment, in addition to the nonzero magnetic moment that is shared with regular magnetic nanoparticles, allows another element of control in the drug delivery process.

$\mathrm{CoFe}_{2} \mathrm{O}_{4}-\mathrm{BaTiO}_{3}$ core-shell MENs of $~ 30 \mathrm{~nm}$ size (Figure 1) have proven to be a successful drug delivery system [59], especially in cancer treatment [60], [61]. Cancer cells have distinctive electrical properties that differ from their healthy counterparts, which MENs are able to interface with. As is characteristic of a rapidly proliferating cellular state, the membrane potential of cancer cells is comparably depolarized (i.e. less negative) [62]. This depolarized membrane potential makes cancer cells more vulnerable to electroporation, allowing contents to be delivered inside the cell through the generated pores [63]. MENs serve as agents of nano-scale electroporation as they respond to an applied magnetic field with the generation of an electric signal, which creates a 
nanoelectroporation effect in close vicinity to the MENs. The nanoelectroporation is highly preferential to cancer cells due to their electric properties: the magnetic field required to generate a significant interaction between the electric systems of the cell membrane and the MENs is orders of magnitude weaker for cancer cells than for healthy cells. This difference in cell membrane properties allows a very weak magnetic field to nanoelectroporate cancer cells but only a very strong field to nanoelectroporate healthy cells, allowing weak fields to be used for cancer treatment in a way that does not affect healthy cells. After step one of entering the cancer cells while avoiding healthy cells is complete, step two would be to release the drug carried on the MENs. Once the MENs have entered the inside of the cell, an increase in the magnetic field strength can induce the MENs to release the drug carried [61]. Both the directing field and higher releasing field are still well below the capacity to electroporate healthy cells. A variety of functionalized coatings are available to fine-tune the strength of binding between the MENs and the desired payload drug to ensure minimal loss prior to arrival at the tumor site, as is directed by one magnetic field strength, and until the manually triggered release, as is dictated by a greater magnetic field strength [64].

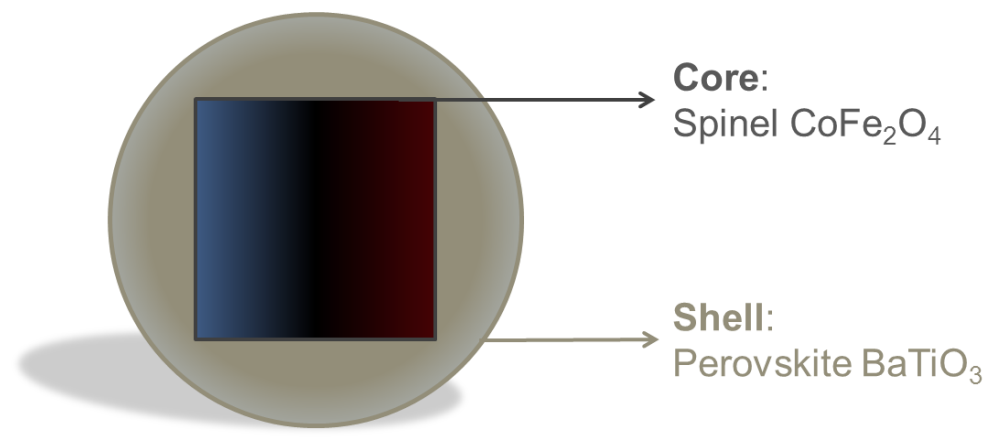

Figure 1. Magnetoelectric Nanoparticle Schematic

A MEN is composed of a polar ferromagnetic metal nanoparticle core surrounded by a magnetoelectric coupling inducing shell. 
The relatively weak strength required to direct MENs and induce nanoelectroporation at the intended destination allows regular magnets to be convenient options for the magnetic field generators [59]. A d.c. field can be amplified and used to also induce the payload release, however, low frequency a.c. magnetic fields are even more effective as the drug release mechanisms [61]. The generic applicability of this approach makes it preferable to drug release approaches relying on very specific cellular microenvironmental conditions at the tumor site to induce making the drug bioavailable upon delivery, as such conditions are more likely to differ from case to case. Likewise, this technique does not require the generation of heat, which could potentially damage surrounding healthy tissue. Furthermore, active targeting molecules can also be utilized to enhance MENs targeting. MENs physical targeting capabilities are complimented by the EPR effect, but do not necessarily require it to function, so MENs have the potential to target tumors too small to establish a significant EPR effect. In this scenario, cancer cells do not need to cluster to establish the targeting effect, as it is intrinsic to each cell in the form of inherent electrical properties, meaning both undetected and metastasized cells can be simultaneously targeted.

\section{$\underline{2.5 \text { Conclusion }}$}

There are many nanoparticle treatment systems both in clinical trials and already being utilized in accordance with FDA regulation [65], and even more still under preliminary investigation with promising results. The future may hold a nanoparticle treatment system for every cancer, perhaps as a hybrid formulation combining multiple particle types carrying a cocktail of drugs for highly personalized therapy. Each 
nanotechnology based approach has strengths and weaknesses. For example, active targeting usually requires a larger particle, while EPR benefits from smaller particles. Furthermore, EPR has been reported to be the more effective targeting agent of the two [66]; however, one of the weaknesses of EPR is that inflamed tissue tends to have similar leaky vasculature to cancer tissues, making an infection while on EPR based treatment more risky to the patient [39]. Similarly, organic nanoparticles degrade comparatively rapidly post-administration, while inorganic particles can carry a payload for an extended period of time; however, inorganic particles are cleared from the organism comparatively slower than organic particles.

There are many aspects to consider when designing a nanomedicine for each cancer case, but the extensive amount of size and formulation options available is an asset. In any case, any nanocarrier offers extensive improvement over free drug treatment, as free drugs have a shorter circulation life and, therefore, need higher doses or prolonged administration to see the desired treatment effect. In the case of cancer, higher drug doses can be nearly fatal from the extensive side effects of healthy tissue receiving the drug. Nanotechnologies offer more accurate drug delivery or even drug free treatment options, along with simultaneous monitoring for vastly improved cancer patient care. We believe that MENs hold the greatest cancer treatment potential, as they are highly specific to cancer cells but also highly generalizable between different cancer types, and offer unprecedented opportunity for externally controlled targeting and payload release along with conventional nanoparticle utilities such as patient monitoring. 


\section{CHAPTER 3: INTRODUCTION TO MENS DRUG DELIVERY \& RELEASE}

\section{$\underline{3.1 \text { Introduction }}$}

This chapter focuses on three previous publications from our lab group that first demonstrate the use of MENs in drug delivery applications. These papers represent the immediate predecessors to this body of dissertation research. The physics and principles we employ have first been described in these publications. Table 2 is a summary of this chapter. The work is presented in chronological order to outline the history and progression that lead us to the starting point of this dissertation project.

Table 2. Preceding publications on MEN drug delivery.

\begin{tabular}{|clcc|} 
Section & \multicolumn{1}{c}{ Paper } & \multicolumn{1}{c}{ Summary } & Reference \\
\hline 3.2 & $\begin{array}{l}\text { Externally controlled on-demand release of } \\
\text { anti-HIV drug using magneto-electric } \\
\text { nanoparticles as carriers }\end{array}$ & $\begin{array}{l}\text { Successful drug } \\
\text { delivery and release } \\
\text { proof of concept }\end{array}$ & [59] \\
\hline $\mathbf{3 . 3}$ & $\begin{array}{l}\text { Magneto-electric Nanoparticles to Enable } \\
\text { Field-controlled High-Specificity Drug } \\
\text { Delivery to Eradicate Ovarian Cancer Cells }\end{array}$ & $\begin{array}{l}\text { Successful cancer } \\
\text { treatment proof of } \\
\text { concept }\end{array}$ & [60] \\
\hline $\mathbf{3 . 4}$ & $\begin{array}{l}\text { Magnetic Field-Controlled Release of } \\
\text { Paclitaxel Drug from Functionalized }\end{array}$ & $\begin{array}{c}\text { Pharmacokinetic } \\
\text { quantifications and } \\
\text { optimization }\end{array}$ & [64] \\
\hline
\end{tabular}

\subsection{MEN HIV Drug Delivery \& Release}

We first demonstrated the unique drug delivery capabilities of MENs by treating HIV in an in-vitro model. In this study, for the first time, we demonstrated that dissipation-free, energy-efficient, and low-field on-demand drug release can be achieved with the MEN platform; this is a great improvement upon conventional drug delivery platforms that lack a discrete control over the drug release portion of drug delivery. Figure 2 demonstrates the proposed theory of the physics behind the drug release mechanism that is unique to MENs. The HIV medication AZTTP is covalently and/or 
ionically tagged onto each MEN, facilitated by the surface charge of the MENs being positive and the surface charge of the AZTTP being negative. Then, to release the medication when desired, a magnetic field is externally applied. The magnetic field directs a magnetic dipole moment within the MEN, which causes a strain on the bonds between the MEN and the payload relative to the orientation of this dipole. An alternating current field would repeat this process until both the forward and backward directions have been strained into releasing the payload.
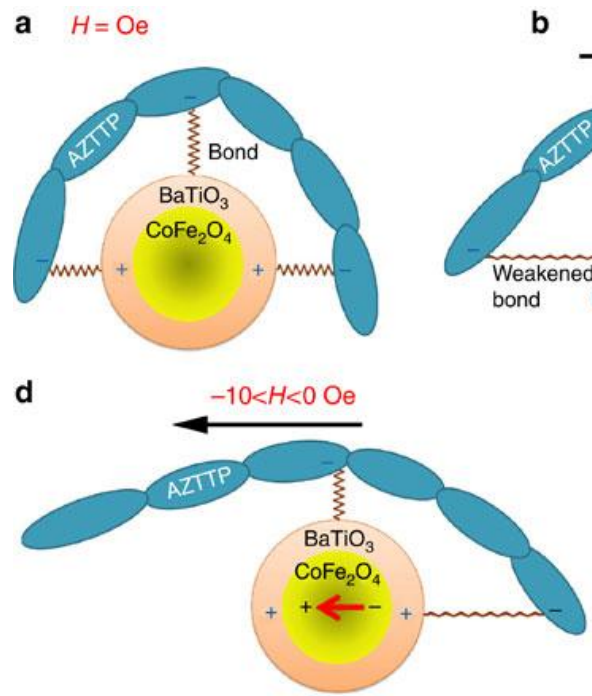

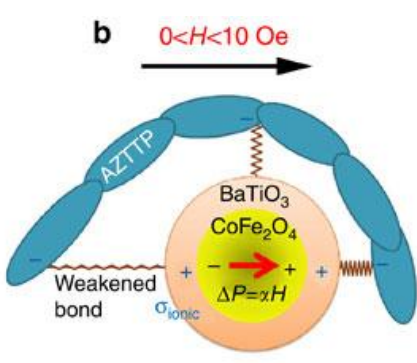

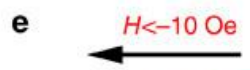

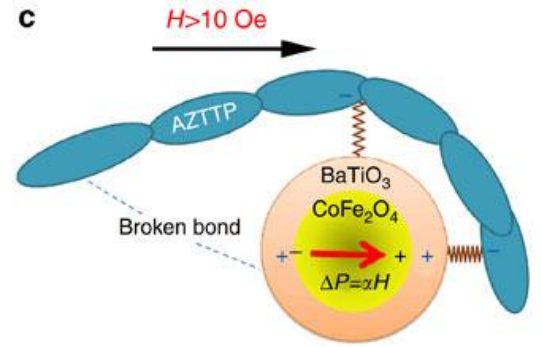

num Regular ionic bond

WMum Field-enhanced bond

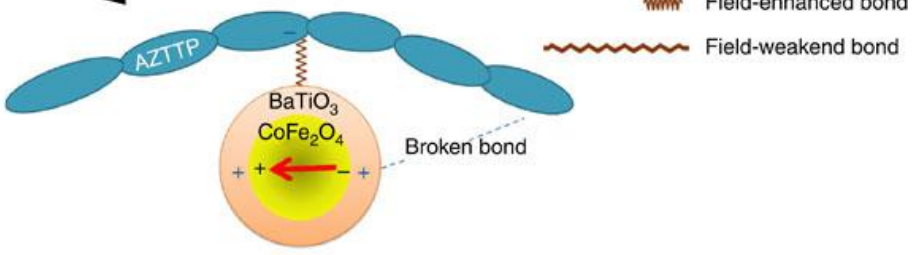

Figure 2. Illustration of the underlying physics of the a.c.-field-triggered release.

A simplified (one directional) illustration of the concept of on-demand drug (AZTTP) release by MENs stimulated by a uniform a.c. magnetic field in X direction. (a) At zero field, only the ionic charge is present in the MEN shell. (b) An additional dipole moment (proportional to the magnetic field) breaks the original symmetry of the charge distribution in the shell. (c) As the field is increased above the threshold value $\left(\sigma_{\text {ionic }} \sim \sigma_{\mathrm{ME}}\right)$, the bond on one side is broken. $(\mathrm{d}, \mathrm{e})$ The field is reversed to break the bond on the opposite side of the nanoparticle. The red arrows show the electric dipole due to the ME effect. In practice, owing to the random configurations of nanoformulations with respect to the field, the effect is present along every central bond orientation. 
This drug release mechanism is demonstrated in practice in Figure 3. AZTTP was mostly able to stay on the MENs at a field of 12 Oe in both a.c. and d.c. contexts. This ensures that ambient magnetic fields do not trigger the drug release. The difference between d.c. and a.c. field application on drug release is very clear around 44 Oe, where the d.c. field released only $16.4 \%$ of the drug, as opposed to $28.5 \%$ with a modest a.c. and $89.3 \%$ with a strong a.c. of $1000 \mathrm{~Hz}$. Interestingly, a strong enough magnetic field, d.c. or a.c., releases nearly $100 \%$ of the drug. This is likely due to the bond-breaking side of the drug chain gaining enough momentum and causing the chain to break free from the MEN shell even at zero frequency when the applied field is strong enough.

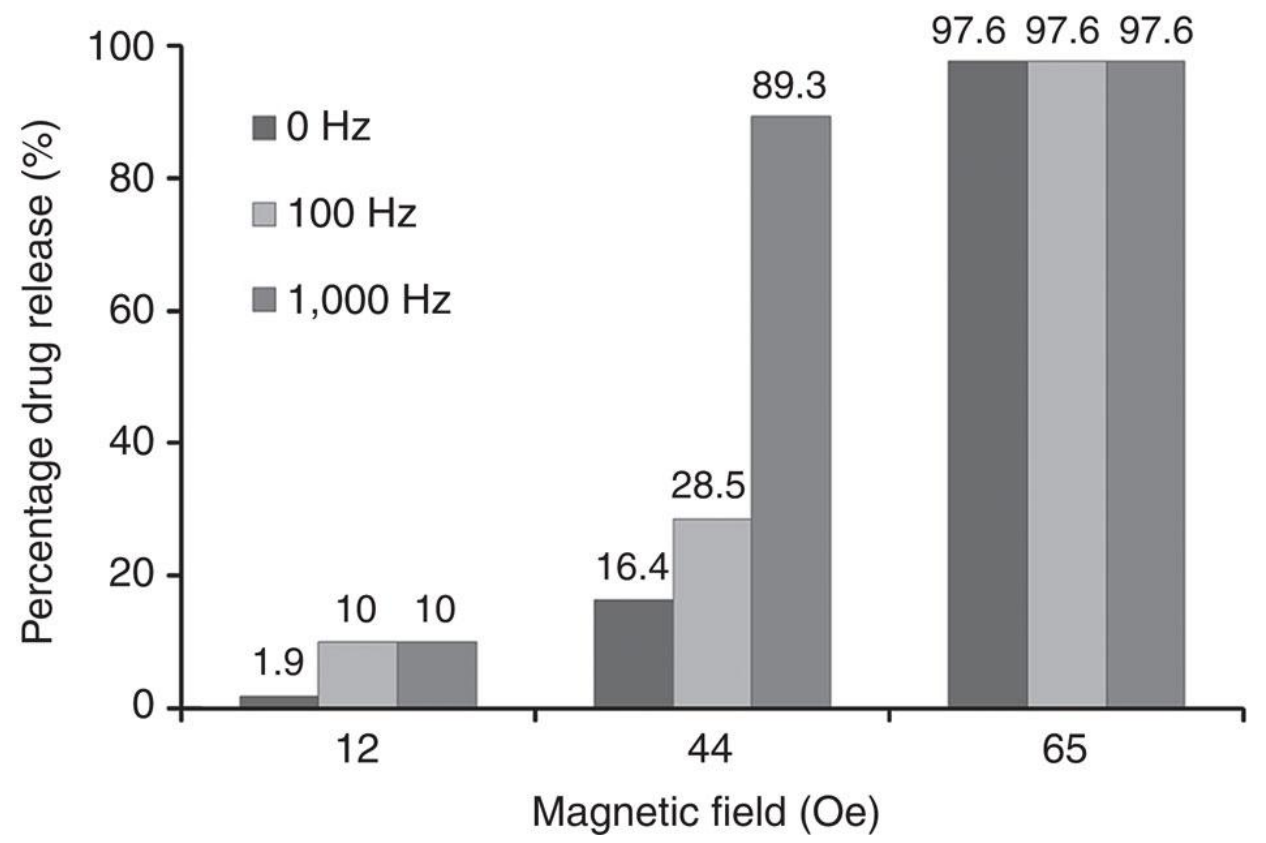

Figure 3. Field strength and frequency dependence of the drug release.

Chart showing the release efficacy of AZTTP drug bound to MENs by a remote magnetic field at different amplitudes and frequencies. 
The duration of the magnetic field exposure also determines how much of the payload is released from the MENs, as seen in Figure 4. The exact percentages are presented in Table 3. With a strong enough field, the drug release is essentially instantaneous, whereas a weak field or intermediate field allows for a slower, timed release. The ability to control how much of the payload is released and when allows for a fine-tuning of the treatment; users can opt for an instantaneous burst release or a slow and steady release for a gradual increase or even maintaining a constant bioavailability to match the rate at which the drug is utilized by the system. There are certainly more field strength/frequency/duration combinations that can utilized to achieve almost any drug release profile with appropriately calculated doses.

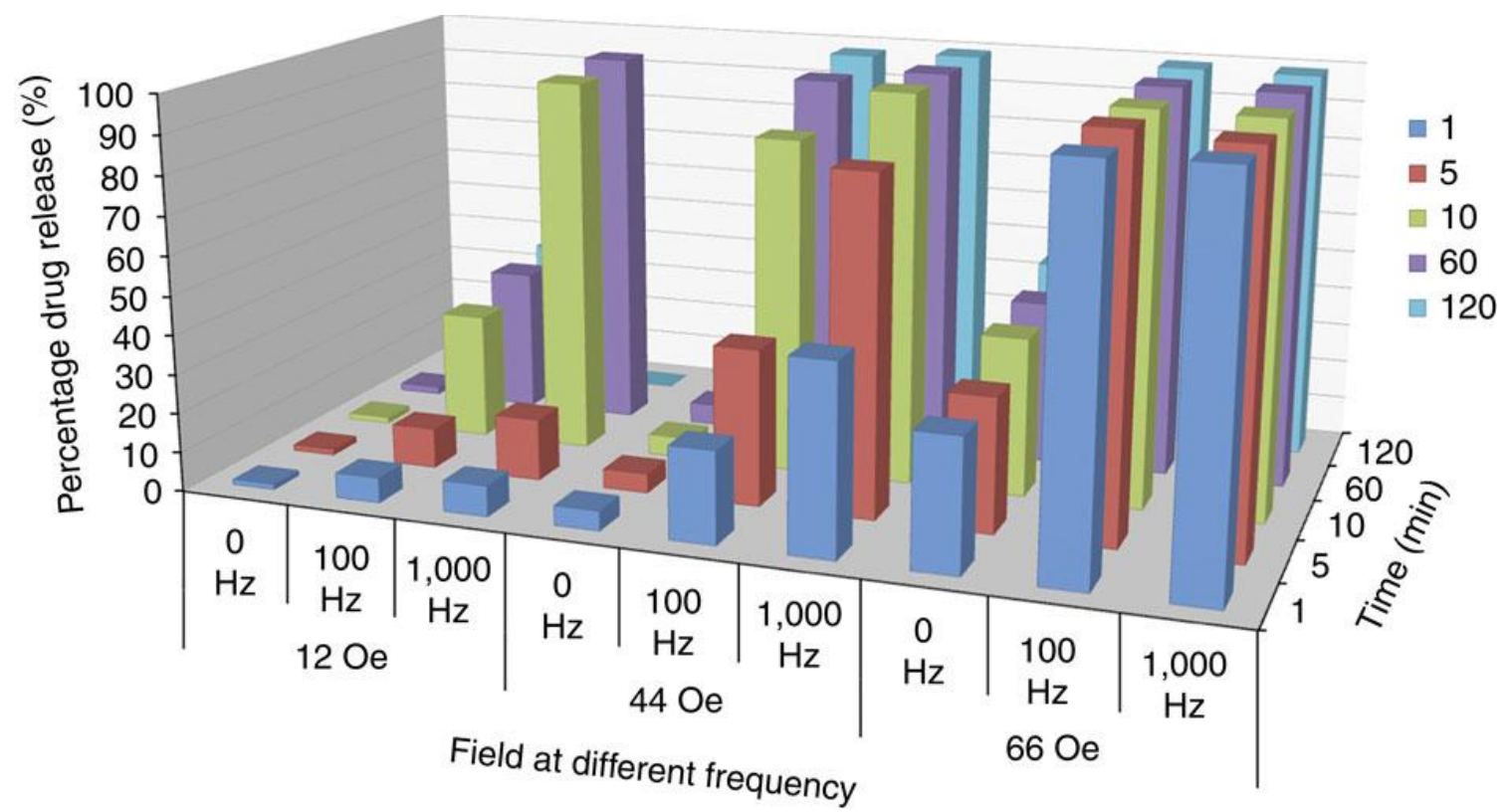

Figure 4. Magnetic field strength and frequency over time determines payload release Pharmacokinetics study results as a three-dimensional chart representation of the drug release percentage at various combinations of the field strength $(12,44$, and $66 \mathrm{Oe})$, the frequency $(0,100$, and $1000 \mathrm{~Hz})$ and the treatment duration $(1,5,10,60$, and $120 \mathrm{~min})$. 
Table 3. Quantification of MENs drug release in a magnetic field over time.

\begin{tabular}{|c|c|c|c|c|c|c|}
\hline \multirow{2}{*}{ Field } & \multirow{5}{*}{ Frequency } & \multicolumn{5}{|c|}{$\begin{array}{c}\text { Drug Release Percentage for Different Field Treatment } \\
\text { Durations (minutes) }\end{array}$} \\
\cline { 2 - 7 } & & $\mathbf{1}$ & $\mathbf{5}$ & $\mathbf{1 0}$ & $\mathbf{6 0}$ & $\mathbf{1 2 0}$ \\
\hline \multirow{3}{*}{$12 \mathrm{Oe}$} & $0 \mathrm{~Hz}$ & $1.31 \%$ & $1.50 \%$ & $1.52 \%$ & $1.87 \%$ & $1.81 \%$ \\
\cline { 2 - 7 } & $100 \mathrm{~Hz}$ & $6.40 \%$ & $10.14 \%$ & $32.20 \%$ & $36.80 \%$ & $37.89 \%$ \\
\cline { 2 - 7 } & $1000 \mathrm{~Hz}$ & $7.81 \%$ & $15.73 \%$ & $96.20 \%$ & $98.01 \%$ & $98.7 \%$ \\
\hline \multirow{3}{*}{$4 \mathbf{O} \mathbf{O e}$} & $0 \mathrm{~Hz}$ & $5.01 \%$ & $4.90 \%$ & $5.10 \%$ & $5.32 \%$ & $5.41 \%$ \\
\cline { 2 - 7 } & $100 \mathrm{~Hz}$ & $23.30 \%$ & $39.58 \%$ & $85.60 \%$ & $95.66 \%$ & $98.00 \%$ \\
\cline { 2 - 7 } & $1000 \mathrm{~Hz}$ & $47.77 \%$ & $85.33 \%$ & $99.01 \%$ & $99.15 \%$ & $99.31 \%$ \\
\hline \multirow{3}{*}{$66 \mathbf{O e}$} & $0 \mathrm{~Hz}$ & $33.30 \%$ & $33.90 \%$ & $40.23 \%$ & $41.60 \%$ & $44.77 \%$ \\
\cline { 2 - 7 } & $100 \mathrm{~Hz}$ & $98.05 \%$ & $99.21 \%$ & $98.71 \%$ & $99.21 \%$ & $99.27 \%$ \\
\cline { 2 - 7 } & $1000 \mathrm{~Hz}$ & $98.58 \%$ & $97.65 \%$ & $98.37 \%$ & $99.31 \%$ & $99.11 \%$ \\
\hline
\end{tabular}

Finally, the drug delivery platform must be confirmed to be effective in practice. In this demonstrative study, the MENs are used to deliver intact AZTTP past an in-vitro model blood brain barrier (BBB) to HIV infected brain cells. In order for a drug delivery system to be effective, the carrier must not damage or otherwise alter the payload. Figure 5 shows atomic force scanning probe microscopy images of the MENs and the AZTTP individually, bound together, and after magnetic field triggered separation. The nanoformulation did not alter the carried drug, which is further confirmed by the in-vitro study treating active HIV in human peripheral blood mononuclear cells (PBMCs) shown in Figure 6. Initially, a cell viability assay confirmed negligible toxicity from the MENs themselves, then, in this BBB model infected with HIV, the nanoformulation was confirmed to be a highly effective drug delivery system. 

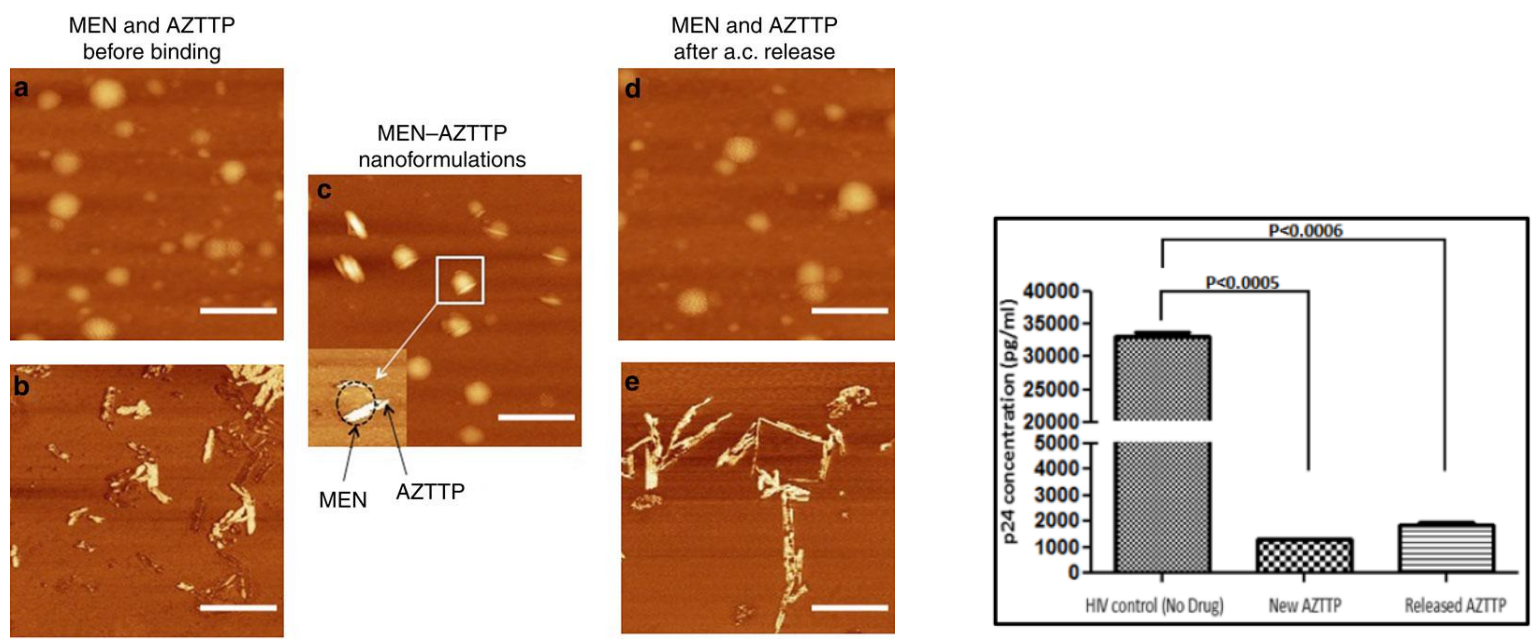

Figure 5. AFM of MENs \& Payload Stages

AFM images at different stages of the release process: (a) MENs and (b) AZTTP chains before the loading (binding) step, (c) AZTTP-MEN nanoformulations after the loading process, (d) MENs and (e) AZTTP after the drug release by a 44 Oe a.c. field at $1,00 \mathrm{~Hz}$. Scale bar, $100 \mathrm{~nm}$.

\section{Figure 6. HIV Treatment}

P24 demonstrating that the ondemand release does not affect the inhibition efficacy of the drug (AZTTP). The p24 concentration is in units of $\mathrm{pg} / \mathrm{ml}$. 


\subsection{MEN Ovarian Cancer Drug Delivery \& Release}

This was the first study to demonstrate the efficacy of MENs as a cancer treatment. The HIV study previously discussed had successfully demonstrated the drug loading and release capabilities of MENs, but this study applied that drug delivery principle specifically to cancer. For this study, we selected the lethal epithelial ovarian cancer as the prototypical cancer model (in the form of the SKOV-3 cell line) due to the well-known challenges associated with treating this particular cancer in a clinical setting. The gold standard in ovarian cancer treatment is cyto-reductive surgery followed by paclitaxel (PTX), a mitotic inhibitor (this class of drugs is explained in Chapter 2.2.1), combination chemotherapy. Our nanoformulation addresses the limited specificity of drug uptake by the cancer that challenges the gold standard treatment. In this set of experiments, the PTX used is Flutax-2, a fluorescently tagged PTX that allows florescent tracking of the movement of the drug. Furthermore, we compare the efficacy of the newer MENs against conventional magnetic nanoparticles (MNs). MNs (discussed in Chapter 2.4.2) serve as a control for the function of the magnetic aspects of the nanoparticles versus the magneto-electric properties unique to MENs. Additionally, we coat the MENs in a thin layer of glycerol mono-oleate (GMO) to achieve a specific functionalization (discussed in detail in Chapter 3.4). Finally, it is important to note that the principles demonstrated in this study are not limited to ovarian cancer; the physics-based nature of the MEN cancer treatment approach means that it will be applicable to almost any cancer.

As introduced in Chapter 2.4, MENs are able to interface preferentially with cancer cells due to the difference in electric cell membrane properties between cancer 
cells and healthy cells, as shown in figure 7 . With no magnetic field applied to induce the dipole moment in the MENs, minimal interaction will occur with all cells (Figure 7a).

However, once a small magnetic field is applied, the MENs respond with the generation of a small electric field that will induce a nanoeletroporation effect at the surface of the cell directly where the nanoparticle is located (Figure 7b). Once internalized, or at least preferentially located at the vicinity of cancer cells, the applied magnetic field can be adjusted to trigger the release of the carried payload by means of bond breakage (Figure 7c and as shown in Chapter 3.2).

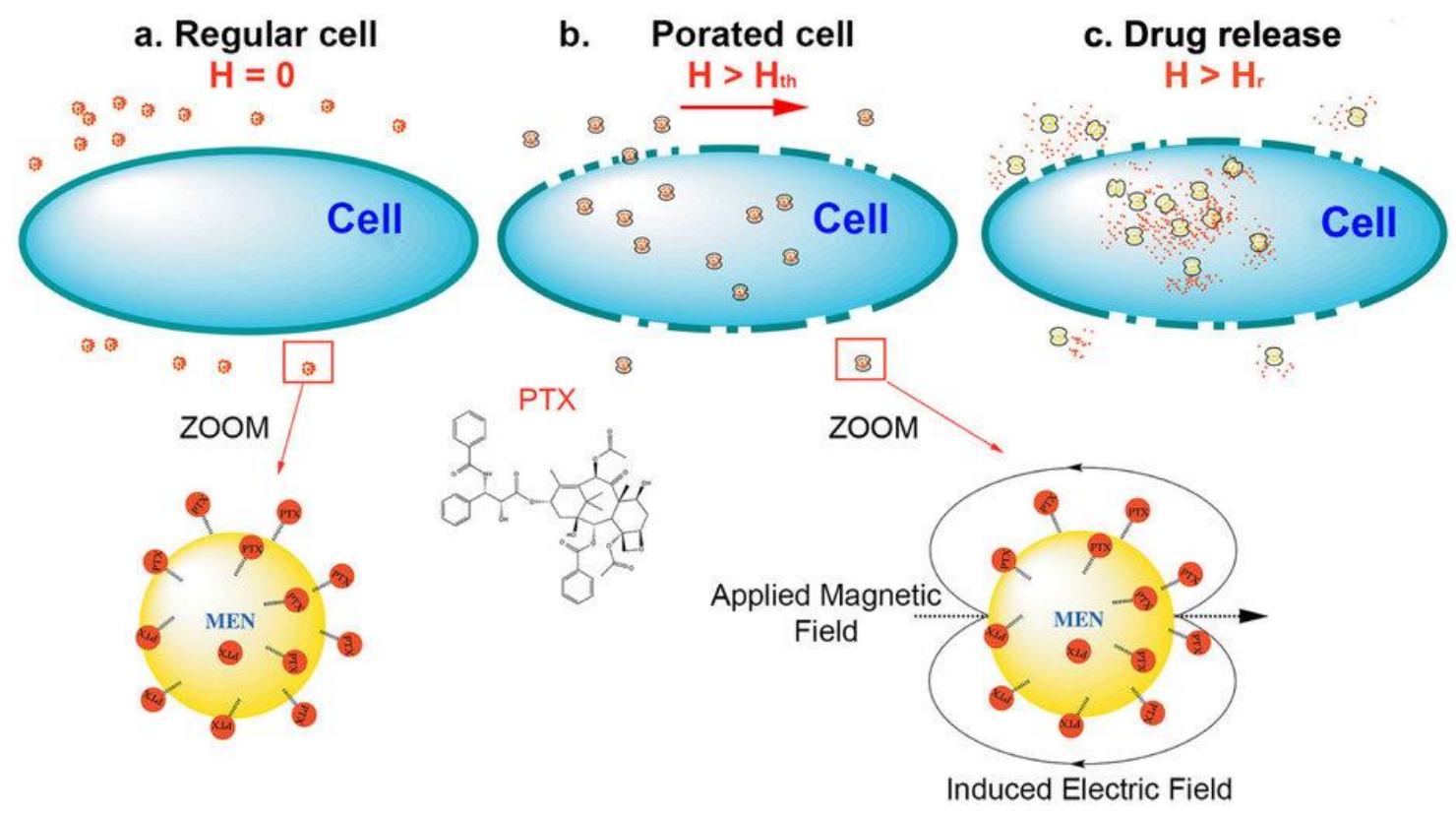

Figure 7. Cancer Nanoelectroportation.

Hypothesis illustration: MENs as field-controlled nanoelectroporation sites to let the drug through the cancer cell membranes. (a) When no magnetic field is applied, there is minimal interaction between the MENs and cells. (b) Once a magnetic field larger than the threshold field required to electroporate $\left(\mathrm{H}_{\text {th }}\right)$ the cell is applied, small pores open up at the cell surface in the immediate vicinity of the MENs by means of nanoelectroporation, thus allowing them entry. (c) When the applied magnetic field is increased to above the nanformualtion release threshold $\left(\mathrm{H}_{\mathrm{r}}\right)$, the MENs release the carried PTX into the cancer cell. 
The first question that must be addressed is whether the MENs nanoformulation is significantly more effective than free-drug uptake and than conventional MN nanoformulation uptake by cancer cells, and Figure 8 shows MENs platform surpassing the other treatments. The MN-PTX nanoformulation as well as the MEN nanoformulations, but with the absence of a magnetic field to stimulate the release, performed no better than free-drug application, as expected. This is because the conventional MNs lack the electroporation effect of the MENs and are therefore unable to readily enter the cells. On the other hand, the MENs formulation holds on to the drug tightly prior to the externally triggered release, as per design (Figure 9 and Table 4). This is clearly demonstrated by the high uptake of the drug when a field is applied to the MENs nanoformulation. Compared to an antibody-based targeted delivery (a popular approach), which shows some improvement over free-drug administration, the MENs stimulated by a magnetic field increased the uptake by a factor of 5 (Figure 8).

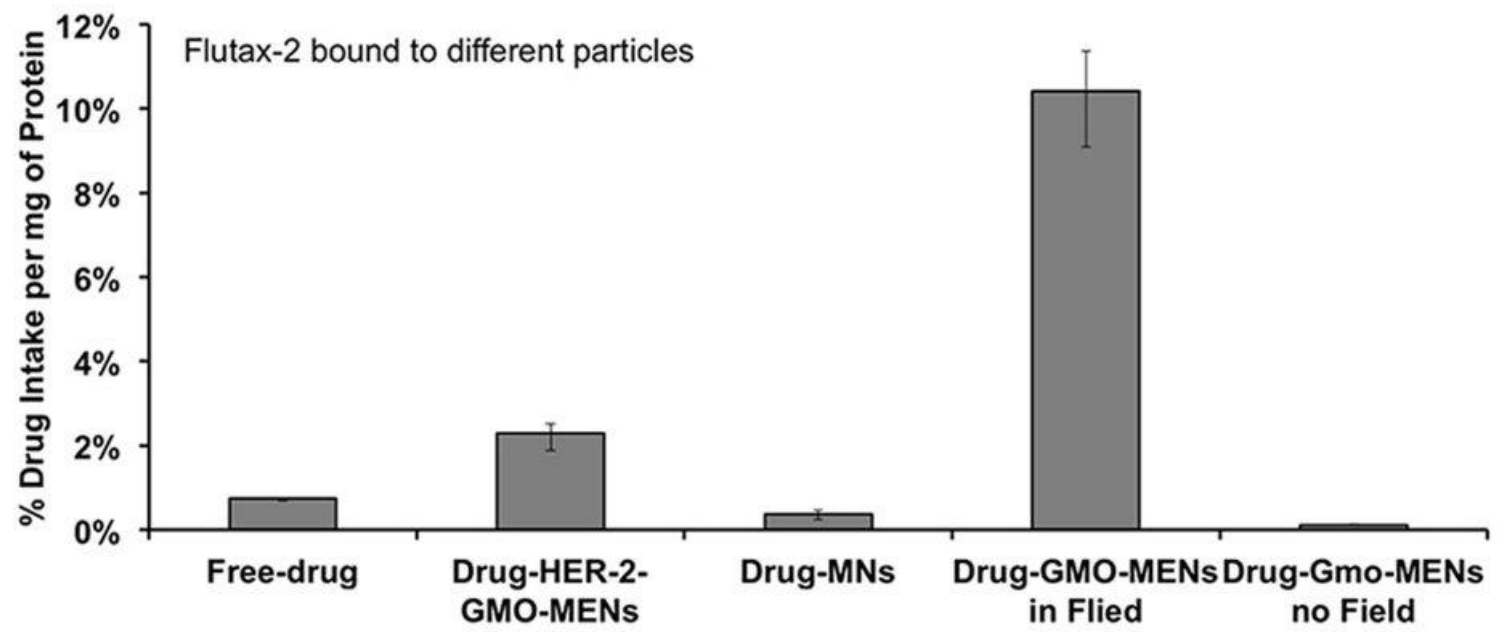

Figure 8. Free drug vs MN vs MEN formulation uptake by cancer cells. Minimal drug made it inside the cell with the free-drug, MN formulation, and MENs formulation in the absence of a magnetic field. The MENs in a magnetic field showed the highest drug delivery percentage, surpassing an antibody targeted MEN formulation by a factor of 5 . 

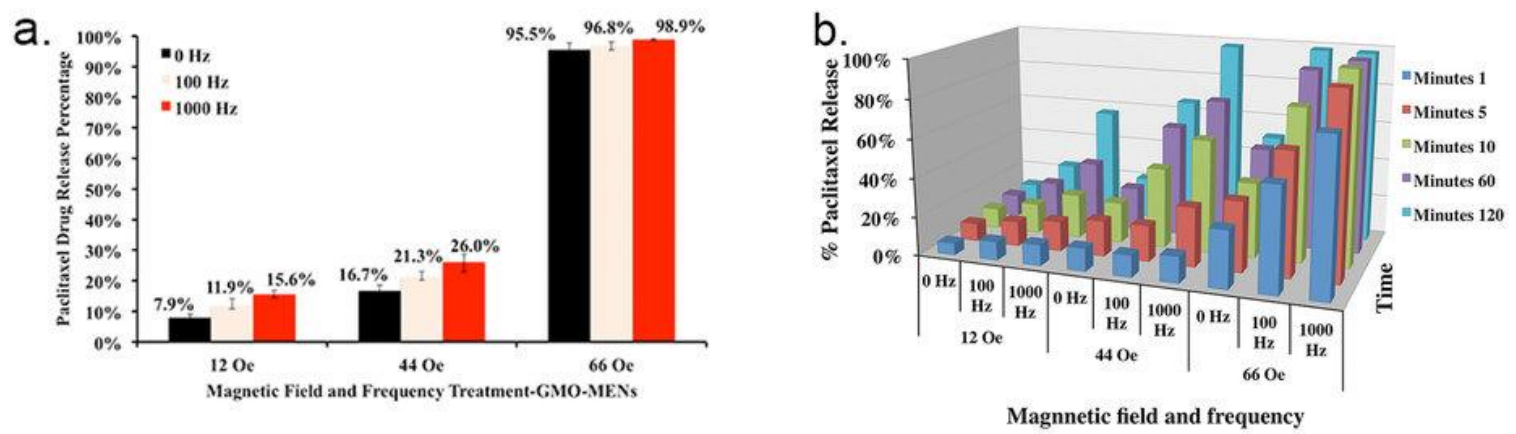

Figure 9. GMO-MENs Release Kinetics.

(a) Chart showing the release efficacy of PTX bound to GMO-coated MENs by a remote magnetic field at different amplitudes and frequencies. (b) Three-dimensional chart representation of the drug release percentage at various combinations of the field strength $(12,44$, and $66 \mathrm{Oe})$, the frequency $(0,100$, and $1000 \mathrm{~Hz})$ and the treatment duration $(1,5,10,60$, and $120 \mathrm{~min})$.

Table 4. Quantification of GMO-MENs drug release in a magnetic field over time.

\begin{tabular}{|c|c|c|c|c|c|c|}
\hline \multirow{3}{*}{$\begin{array}{c}\text { Field } \\
\text { Strength }\end{array}$} & \multirow{2}{*}{$\begin{array}{c}\text { Frequenc } \\
\text { y }\end{array}$} & \multicolumn{5}{|c|}{$\begin{array}{c}\text { Percentage Paclitaxel Release at Different } \\
\text { Treatment Time Durations (Minutes) }\end{array}$} \\
\cline { 3 - 7 } & & 1 & 5 & 10 & 60 & 120 \\
\hline \multirow{3}{*}{$12 \mathrm{Oe}$} & $0 \mathrm{~Hz}$ & $6.1 \%$ & $9.1 \%$ & $10.3 \%$ & $11.4 \%$ & $11.1 \%$ \\
\cline { 2 - 7 } & $100 \mathrm{~Hz}$ & $9.5 \%$ & $12.8 \%$ & $15.3 \%$ & $20.5 \%$ & $24.4 \%$ \\
\cline { 2 - 7 } & $1000 \mathrm{~Hz}$ & $10.7 \%$ & $15.4 \%$ & $22.6 \%$ & $33.7 \%$ & $56.7 \%$ \\
\hline \multirow{3}{*}{$44 \mathrm{Oe}$} & $0 \mathrm{~Hz}$ & $11.8 \%$ & $18.3 \%$ & $20.9 \%$ & $22.2 \%$ & $21.1 \%$ \\
\cline { 2 - 7 } & $100 \mathrm{~Hz}$ & $11.2 \%$ & $18.5 \%$ & $41.3 \%$ & $57.9 \%$ & $66.2 \%$ \\
\cline { 2 - 7 } & $1000 \mathrm{~Hz}$ & $13.4 \%$ & $30.4 \%$ & $58.2 \%$ & $73.2 \%$ & $97.8 \%$ \\
\hline \multirow{3}{*}{$66 \mathrm{Oe}$} & $0 \mathrm{~Hz}$ & $29.1 \%$ & $36 \%$ & $38.4 \%$ & $49.8 \%$ & $50.5 \%$ \\
\cline { 2 - 7 } & $100 \mathrm{~Hz}$ & $53.1 \%$ & $62.9 \%$ & $78.5 \%$ & $92.3 \%$ & $98.5 \%$ \\
\cline { 2 - 7 } & $1000 \mathrm{~Hz}$ & $78.1 \%$ & $93.7 \%$ & $98.5 \%$ & $98.2 \%$ & $98.2 \%$ \\
\hline
\end{tabular}

After it was established that the SKOV-3 cancer cells readily localize the GMOMENs-PTX nanoformulation, we compared how the healthy counterpart to these cells, human ovarian microvascular endothelial cells (HOMEC), responded to the same treatment. As expected, because of the intrinsic differences in the cell membrane electrical properties, the healthy cells did not take up the treatment (Figure 10). A magnetic field of 30 Oe is enough to deliver large amounts of PTX into the cancer cells, but not enough to get a significant amount of the drug into healthy cells. 

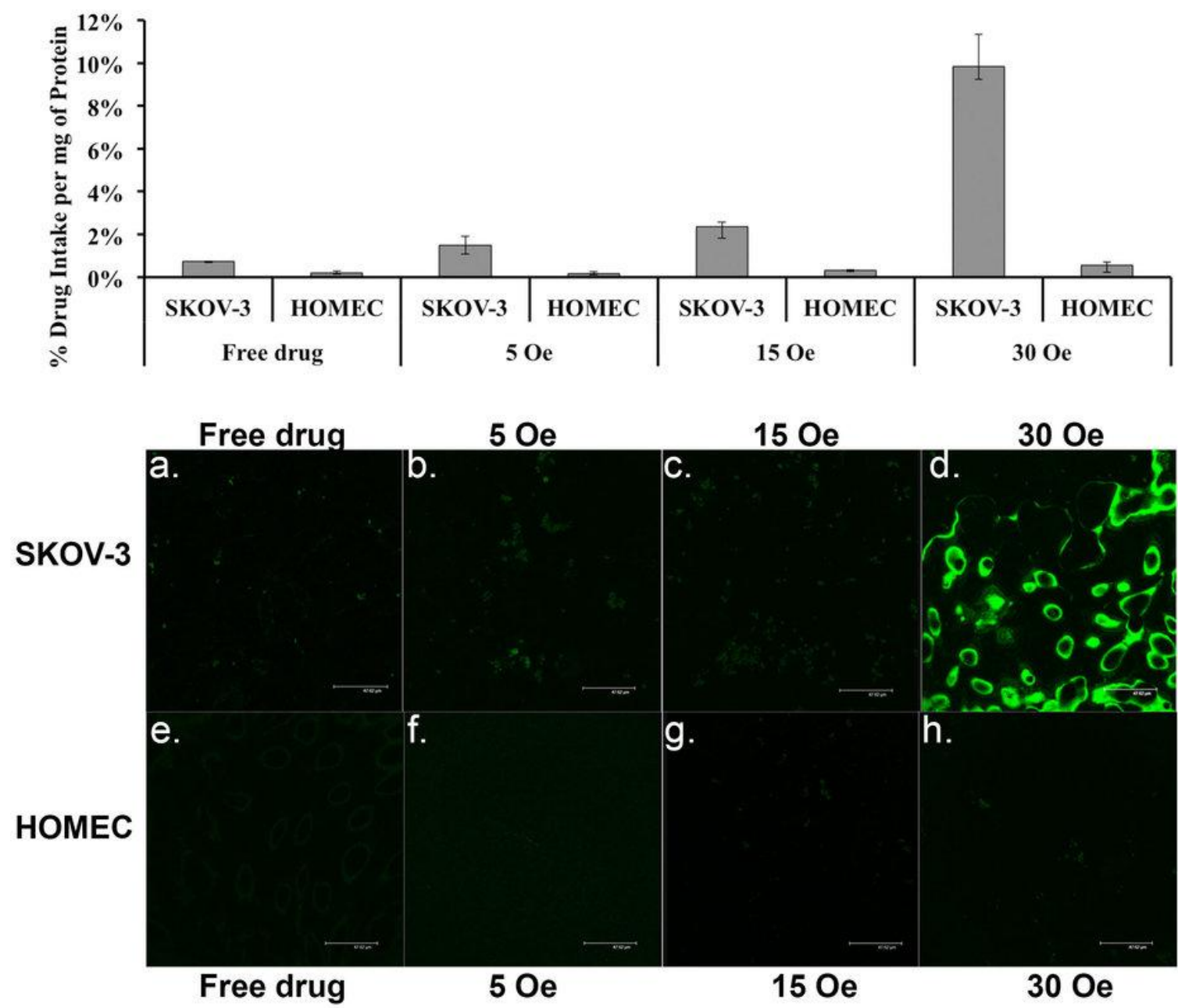

Figure 10. Field dependence of the drug uptake by SKOV-3 and HOMEC cells.

(top) Quantification and comparison of drug uptake between cancerous and healthy ovarian tissue in different magnetic fields. (bottom) Fluorescent microscopy images demonstrating differences in uptake. (a and e) Free drug is minimally taken up, with the cancer cells being more prone to uptake the mitotic inhibitor due to faster mitosis. (b and c) Some uptake is seen with a field applied, increasing with the field, as expected. (d) The critical field at which drug uptake goes up significantly in the cancer cells in around 30 Oe. (f-h) Since the nanoformulation is preventing the drug from being bioavailable and the electroporation threshold for the healthy cells is not in this order of magnitude, essentially no uptake is observed.

Finally, the actual viability of the cancer cells and healthy cells must be observed under this treatment versus control treatments. Figure 11 demonstrates that the GMOMENs-PTX formulation under a magnetic field is extensively more cytotoxic than control formulations. Free drug and HER-2 antibody guided GMO-MENs-PTX (instead of magnetic field guided) treatments show some efficacy, but not nearly as much as the 
magnetic field exposed MENs (Figure 11a). Furthermore, it is also clear that the

cytotoxicity is specifically from the PTX and not from the nanocarrier because cells were fully viable in all of the PTX-free controls (Figure 11b). Table 5 shows the tryptan-blue cell viability assay quantification of the treatments visualized by the confocal microscopy in Figure 11.

a. SKOV-3 cells: Drug (PTX) in Different formulations

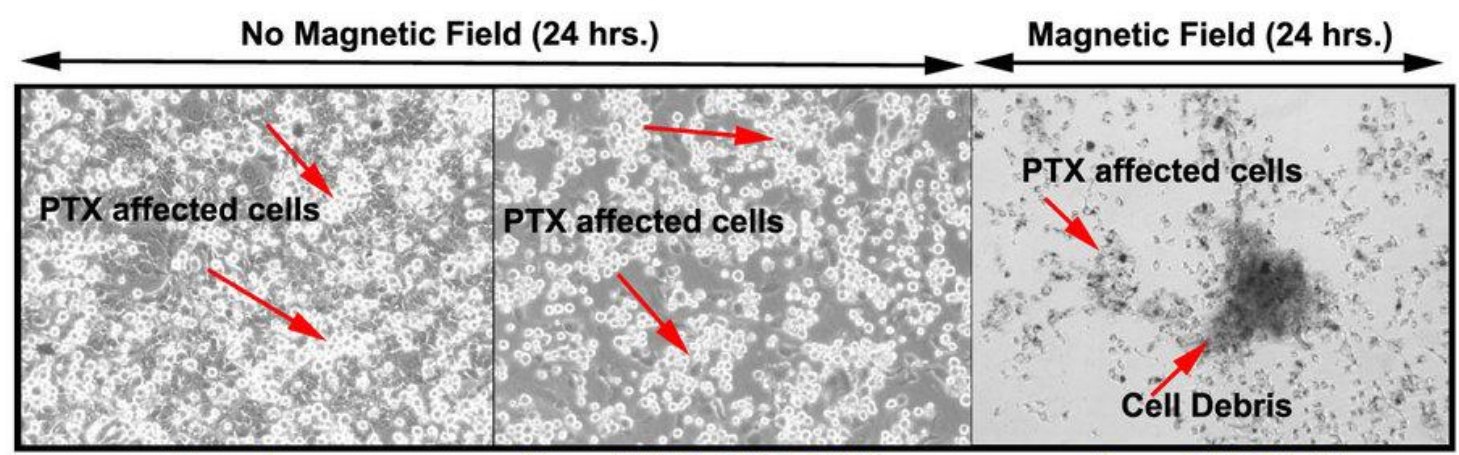

Free drug

Drug-HER2-GMO-MENs

Drug-GMO-MENs

b. SKOV-3 cells: No Drug (PTX) Controls

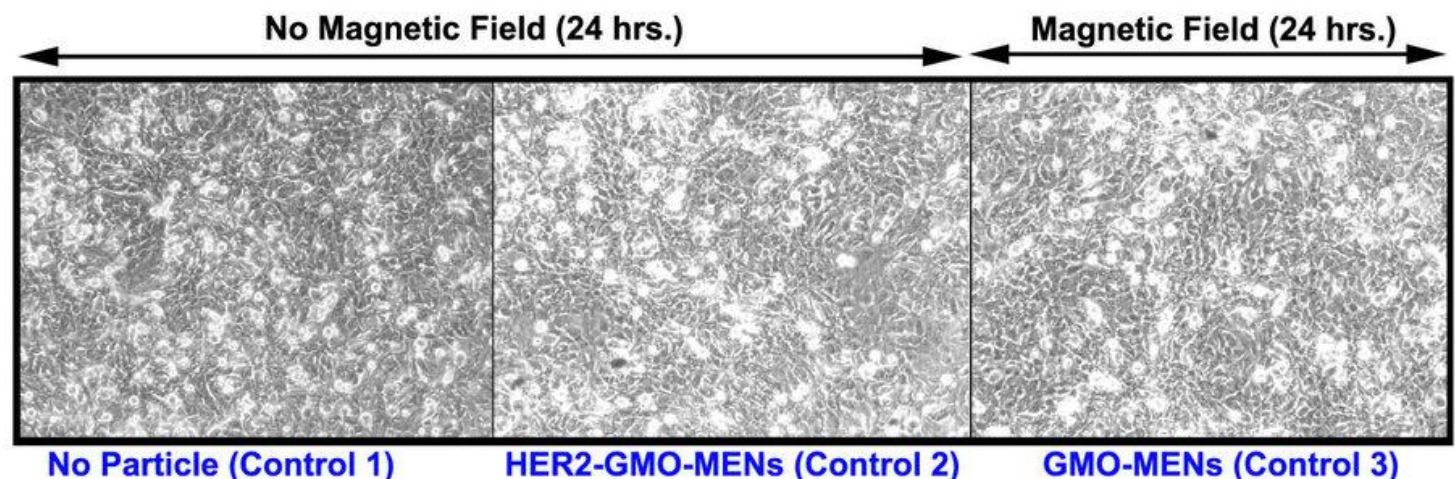

Figure 11. Confocal imaging of SKOV-3 cell viability after treatment by different drug-carrier combinations with and without field.

(a) An applied magnetic field is required to successfully treat the cancer cells, as treatment by free PTX and PTX loaded onto GMO-MENs directed by HER-2 antibodies achieve only minimal cytotoxicity. (b) No significant toxicity can be seen in the PTX-free controls. 
Table 5. Trypan-Blue cell viability assay for nanoformulations and controls.

\begin{tabular}{|c|c|c|c|c|}
\hline Drug & $\begin{array}{c}\text { Field }(30 \\
\mathrm{Oe})\end{array}$ & $\begin{array}{l}\text { Type of } \\
\text { cell }\end{array}$ & Type of Treatment & Percentage Cells Alive \\
\hline \multirow{3}{*}{-} & - & SKOV-3 & $\begin{array}{c}\text { Control-1 } \\
\text { (No particle) }\end{array}$ & $99 \pm 1 \%$ \\
\hline & - & SKOV-3 & $\begin{array}{c}\text { Control-2 } \\
\text { (GMO-MENs-HER2) }\end{array}$ & $98 \pm 1 \%$ \\
\hline & + & SKOV-3 & $\begin{array}{c}\text { Control-3 } \\
\text { (GMO-MENs) }\end{array}$ & $98.5 \pm 0.7 \%$ \\
\hline \multirow{5}{*}{+} & - & SKOV-3 & FREE & $86 \pm 8.8 \%$ \\
\hline & - & SKOV-3 & GMO-MENS-HER2 & $71 \pm 9.8 \%$ \\
\hline & + & SKOV-3 & GMO-MENS & $31 \pm 11.8 \%$ \\
\hline & + & SKOV-3 & GMO-MENs & $\begin{array}{c}33.8 \pm 9.3 \%(24 \text { hrs. }) \\
9.7 \pm 4.1 \%(36 \text { hrs. })\end{array}$ \\
\hline & + & HOMEC & GMO-MENs & $\begin{array}{l}94.9 \pm 2.4 \% \text { ( } 24 \text { hrs. }) \\
84.1 \pm 7.8 \% \text { (36 hrs. })\end{array}$ \\
\hline
\end{tabular}

\subsection{MENs Drug Delivery \& Release Optimization}

This study describes the optimization process that our MENs underwent to establish the ideal functionalization and applied field settings to achieve near perfect delivery and release capabilities.

Most nanoparticles have the capacity to be surrounded by a different material than they are composed of to establish new properties at the particle's surface. These properties determine attributes like drug loading, transportation throughout the biological system, and drug release. Some of the most common surface modifying molecules include the small natural homopolymer poly-L-lysine polysorbate surfactant Tween-20, non-ionic oil soluble/dispersible type surfactant GMO, and the water-soluble ethyl-3-(3dimethylaminopropyl) carbodiimide (EDC). PLGA is another option, and it can also be used as the exclusive component of the nanoparticle (Chapter 2.3). The type of surface 
modification used would depend on the desired profile of drug binding and release kinetics.

Previously, drug release from nanocarriers has depended on subtle changes in the microenvironment of the destination tissue, such as cleavage by site-specific enzymes, temperature changes, $\mathrm{pH}$ changes, redox reaction established microenvironements, etc. Now that MENs have opened the door to externally controlled on-demand drug release with the application of magnetic fields, as demonstrated in the two previously described studies, the exact drug-release profile can be further tailored to each medical scenario with surface modification. For instance, if the nanoformulation is destined for a deep tissue that might require an extended period of time for the nanoformulation to accumulate there, EDC would be a good choice for the intermediate layer. The carbodiimide cross-linker intermediate layer would establish a strong covalent bond with the payload drug by reacting with multiple amide and hydroxyl functional groups. In contrast, if a nanoformulation needs to achieve a nearly $100 \%$ release rate and with a comparatively weaker magnetic field, Tween-20 and GMO are the better options for the intermediate layer. These non-ionic surfactants bind their payload drugs via non-covalent interactions that are easier to disrupt with a magnetic field.

The intermediate layer needs to only be approximately $2 \mathrm{~nm}$ thick to grant the functionalization properties that will determine the magnitude and frequency of the magnetic field needed to dissociate the payload drug from the functional layer on the nanoparticle. Figure 12 shows SEM images of clusters of MENs coated in GMO, Tween20, or EDC functional layers; these are the coatings investigated in this study to deliver a PTX payload. Table 6 lists the nanoparticle sizes as well as zeta potentials of the different 
nanoformulations. Zeta potential predicts the binding affinity between nanoparticles and a payload, with a higher zeta potential suggesting a higher dissociation field needed to disrupt the higher Coulomb forces from higher effective charge on the formulation.

Fourier transform infrared (FTIR) absorption spectrum shows the unique bonds between the MENs and the different coatings in Figure 13.

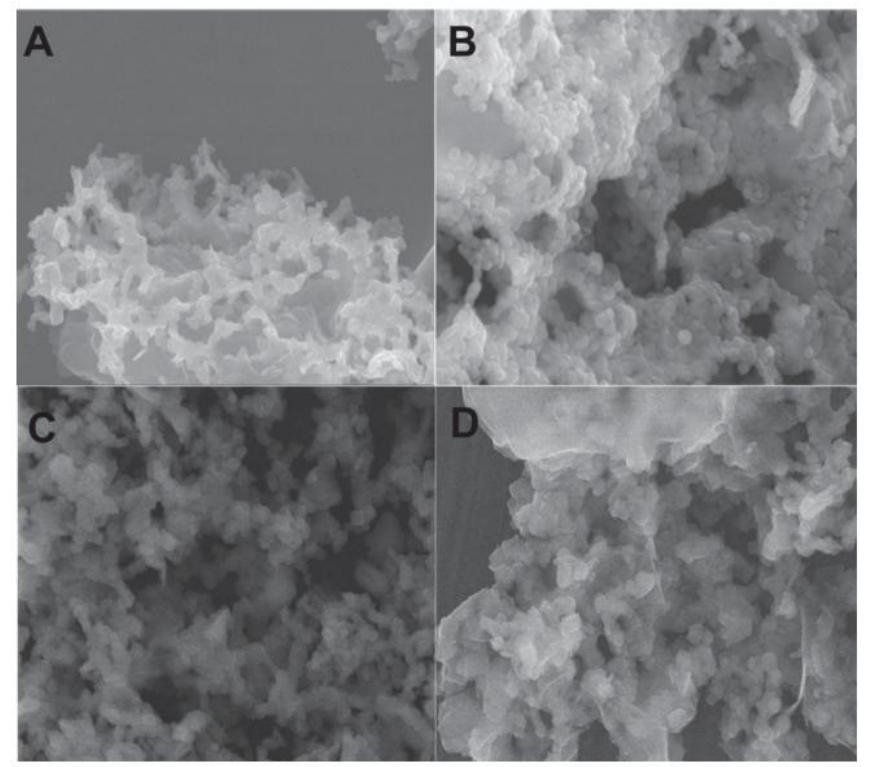

Figure 12. SEM images of MENs with functionalized coatings (a) Uncoated MENs, (b) GMO-MENs, (c) Tween-20-MENs, (d) EDC-MENs. 
Table 6. Nanoparticle size \& zeta potential measurements.

\begin{tabular}{lcc}
\hline Functionalizing agent & $\begin{array}{c}\text { Size } \\
{[\mathrm{nm}]^{\mathrm{a})}}\end{array}$ & $\begin{array}{c}\text { Zeta potential } \\
{[\mathrm{mV}]^{\mathrm{a})}}\end{array}$ \\
\hline Uncoated-MENs & $28.6 \pm 7.5$ & $-45.0 \pm 1.72$ \\
GMO-MENs & $30.9 \pm 8.6$ & $-41.6 \pm 0.26$ \\
Tween-20-MENs & $29.5 \pm 5.6$ & $-34.9 \pm 0.20$ \\
EDC-MENs & $31.1 \pm 4.4$ & $-30.4 \pm 0.81$ \\
PTX-MENs & $28.9 \pm 7.7$ & $-44.8 \pm 1.67$ \\
PTX-GMO-MENs & $44 \pm 6.6$ & $-40.7 \pm 0.1$ \\
PTX-Tween20-MENs & $32.3 \pm 6.1$ & $-31.1 \pm 0.51$ \\
PTX-EDC-MENs & $33.8 \pm 3.2$ & $-27.7 \pm 1.3$ \\
\hline
\end{tabular}

a) Average \pm standard deviation.

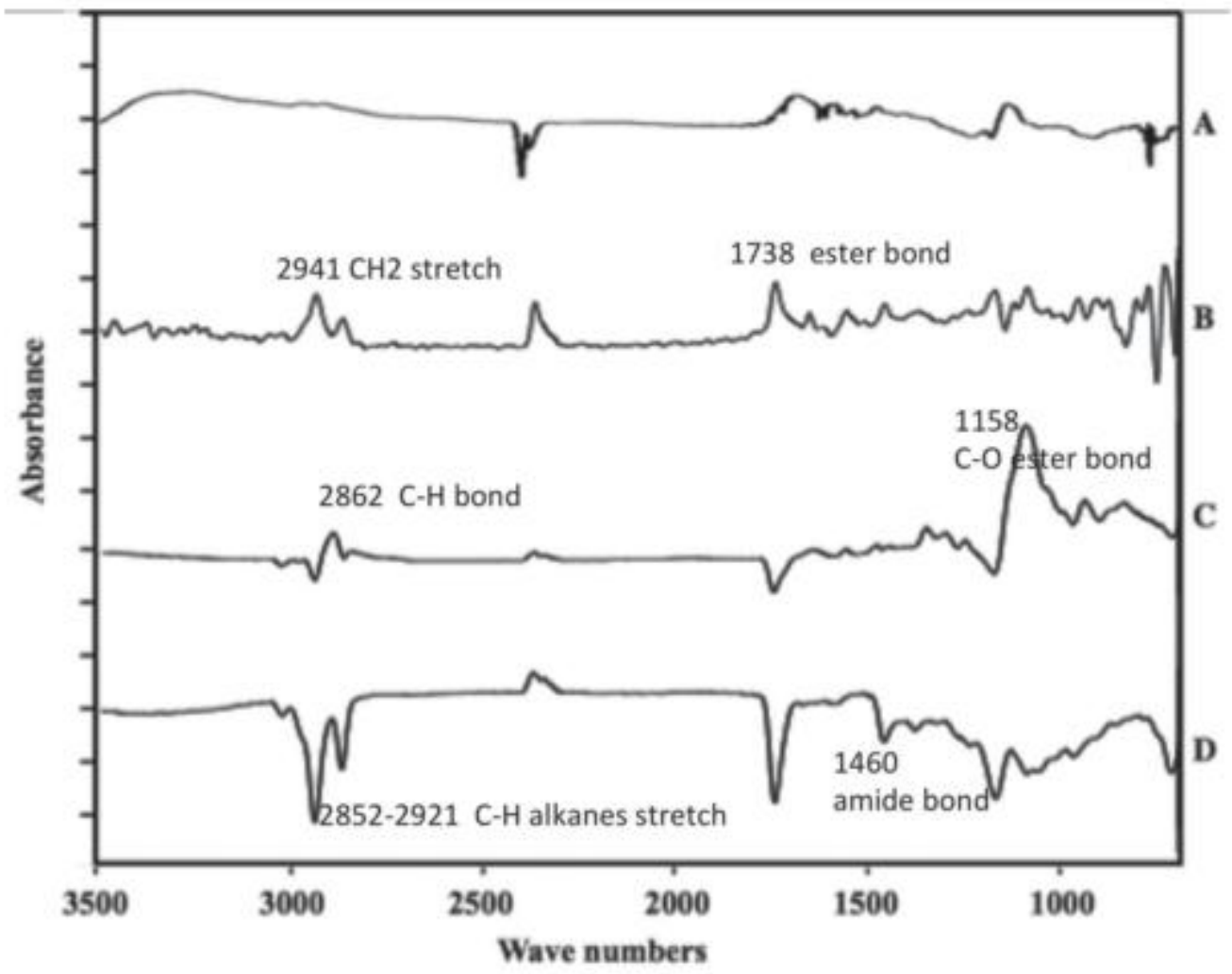

Figure 13. FTIR spectra of MENs with functionalized coatings.

(a) Uncoated MENs, (b) GMO-MENs, as confirmed by the peaks at $1738 \mathrm{~cm}^{-1}$ (ester bond) and $2941 \mathrm{~cm}^{-1}\left(\mathrm{CH}_{2}\right.$ stretching modes), (c) Tween-20-MENs, as confirmed by the peaks at $1158 \mathrm{~cm}^{-1}(\mathrm{C}-$ $\mathrm{O}$ ester bond) and $2862 \mathrm{~cm}^{-1}$ ( C-H bond), (d) EDC-MENs, as confirmed by the peaks at $2852 \mathrm{~cm}^{-1}$ to $2921 \mathrm{~cm}-1$ (C-H alkane stretch) and $1460 \mathrm{~cm}^{-1}$ (amide bonds). 
Aside from the payload release characteristics, the drug loading potential of each functional layer is also an important consideration. After incubating the drug with each nanoformulation, the percent loaded over time is shown in Figure 14. Likewise, the nonstimulated release rate (decay over time) in a biological organism must be considered, and loss of payload in a peristaltic pump model circulatory system was calculated and shown in Table 7.
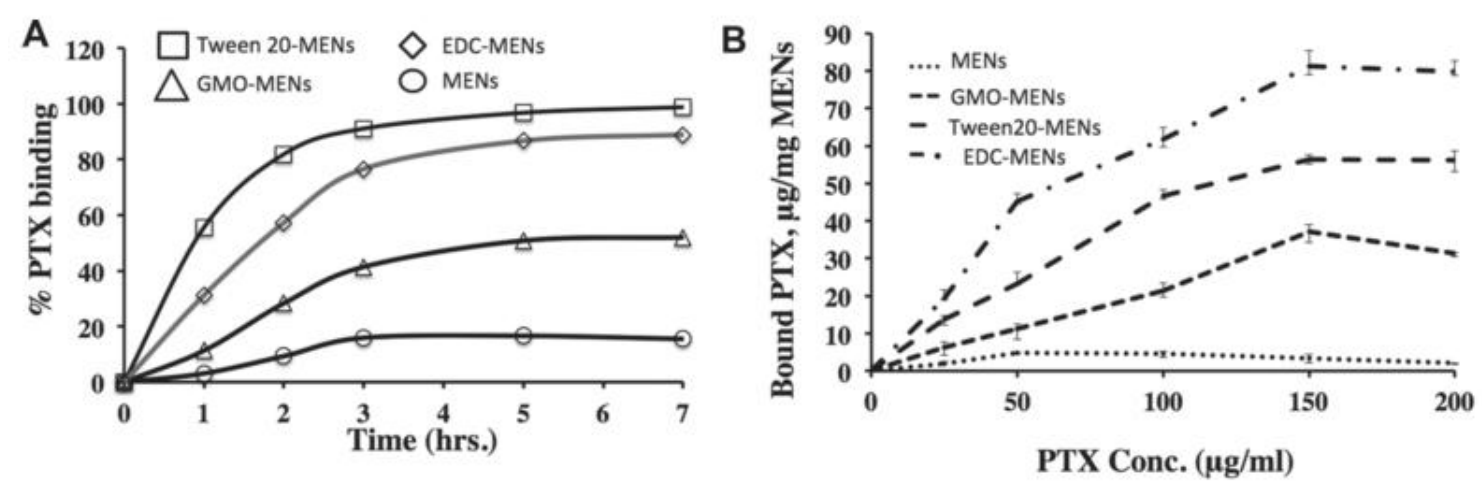

Figure 14. PTX payload loading.

(a) Time kinetics of PTX binding onto MENs nanoformulation surface at a 1:10 weight ratio. (b) Isotherm kinetics of PTX binding onto MENs nanoformulation surface at varying PTX drug concentration; readings taken at 3 hour of incubation time.

Table 7. Intact nanoformulation in peristaltic pump human circulation model ( $8 \mathrm{ml} / \mathrm{min}$ flow for 2 hours).

\begin{tabular}{|c|c|c|c|c|}
\hline Nanoformulation & $\begin{array}{c}\text { Uncoated } \\
\text { MENs }\end{array}$ & GMO-MENs & $\begin{array}{c}\text { Tween-20- } \\
\text { MENs }\end{array}$ & EDC-MENs \\
\hline Intact PTX & $58.4 \%$ & $79.5 \%$ & $87.0 \%$ & $91.1 \%$ \\
\hline
\end{tabular}

The functional layer-determined release kinetics are shown in Figure 15. Notably, non-functionalized MENs most readily release their payload, with a 50 Oe field releasing $77.9,88.8$, and $99.0 \%$ with 0,100 , and $1000 \mathrm{~Hz}$, respectively. On the opposite end of the spectrum, 200 Oe at $1000 \mathrm{~Hz}$ was only able to release 6.5\% of the EDC-MENs' payload. GMO-MENs and Tween-20 MENs fell in-between the two extremes, with the Tween-20 
nanoformulation requiring a substantially stronger field to release large amounts of the carried payload compared to GMO nanoformulation. The GMO-MENs nanoformulation has the advantage of a full range of release under 100 Oe, allowing a very weak magnetic field to release the payload fully in a short and modifiable (with exact strength and frequency) time span.
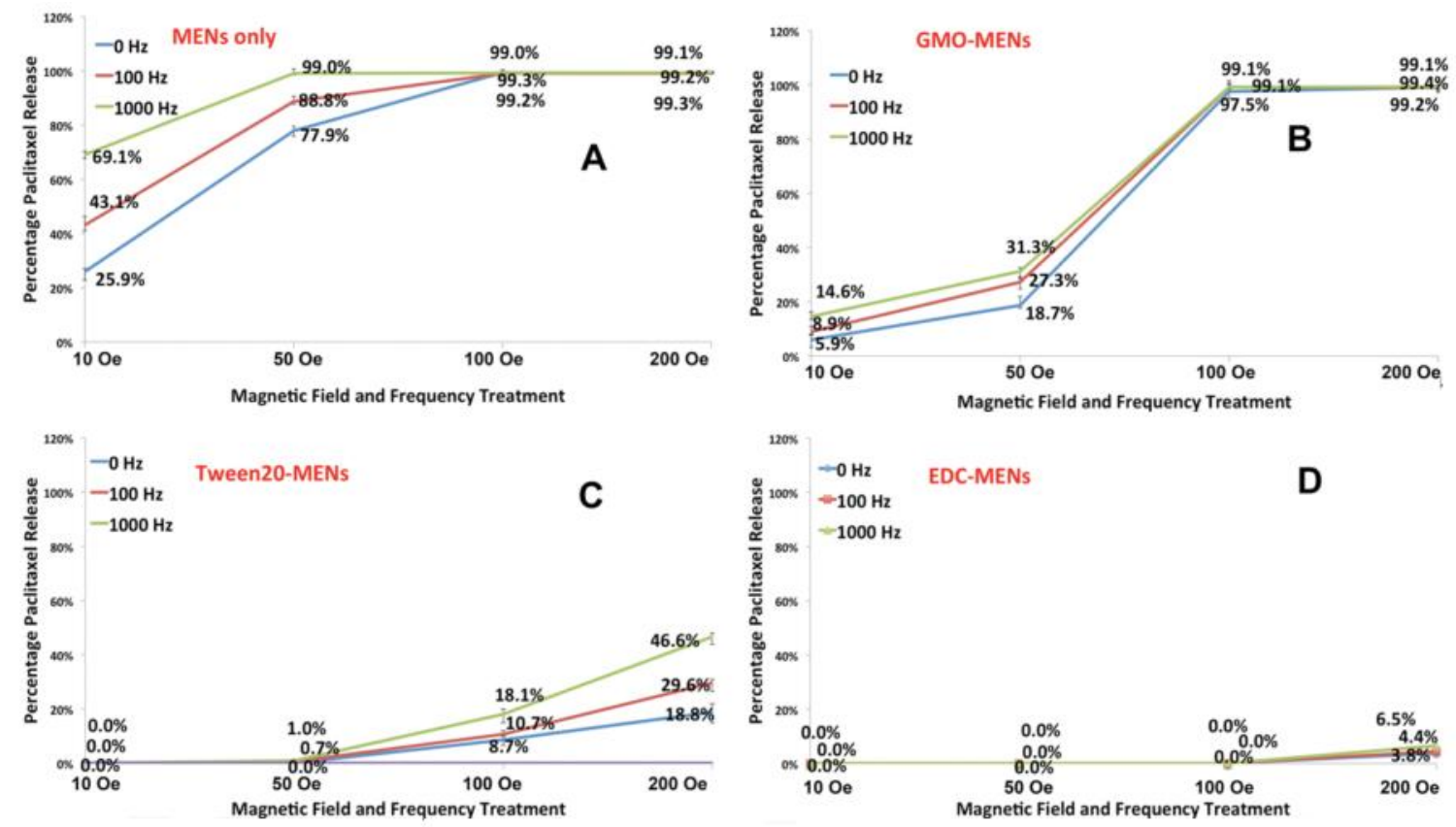

Figure 15. Magnetic field and frequency dependent drug release kinetics from functionalized MENs. (a) Uncoated MENs, (b) GMO-MENs, (c) Tween-20-MENs, and (d) EDC-MENs.

Lastly, the lack of toxicity of these functionalized nanoformulations themselves (as opposed to the payload they carry) must be confirmed. The results of an XTT cytotoxicity assay using SKOV-3 cells are shown in Figure 16. EDC does appear to cause minor cytotoxicity, as well as Tween-20 to a lesser extent. GMO-MENs and nonfunctionalized MENs display no significant toxicity, with GMO-MENs leaving $100 \%$ of the cells viable after exposure. The presence or absence of a magnetic field does not affect the cytotoxicity results. Furthermore, the increase in field frequency consistently 
releases a larger portion of the payload between all three functional coatings.

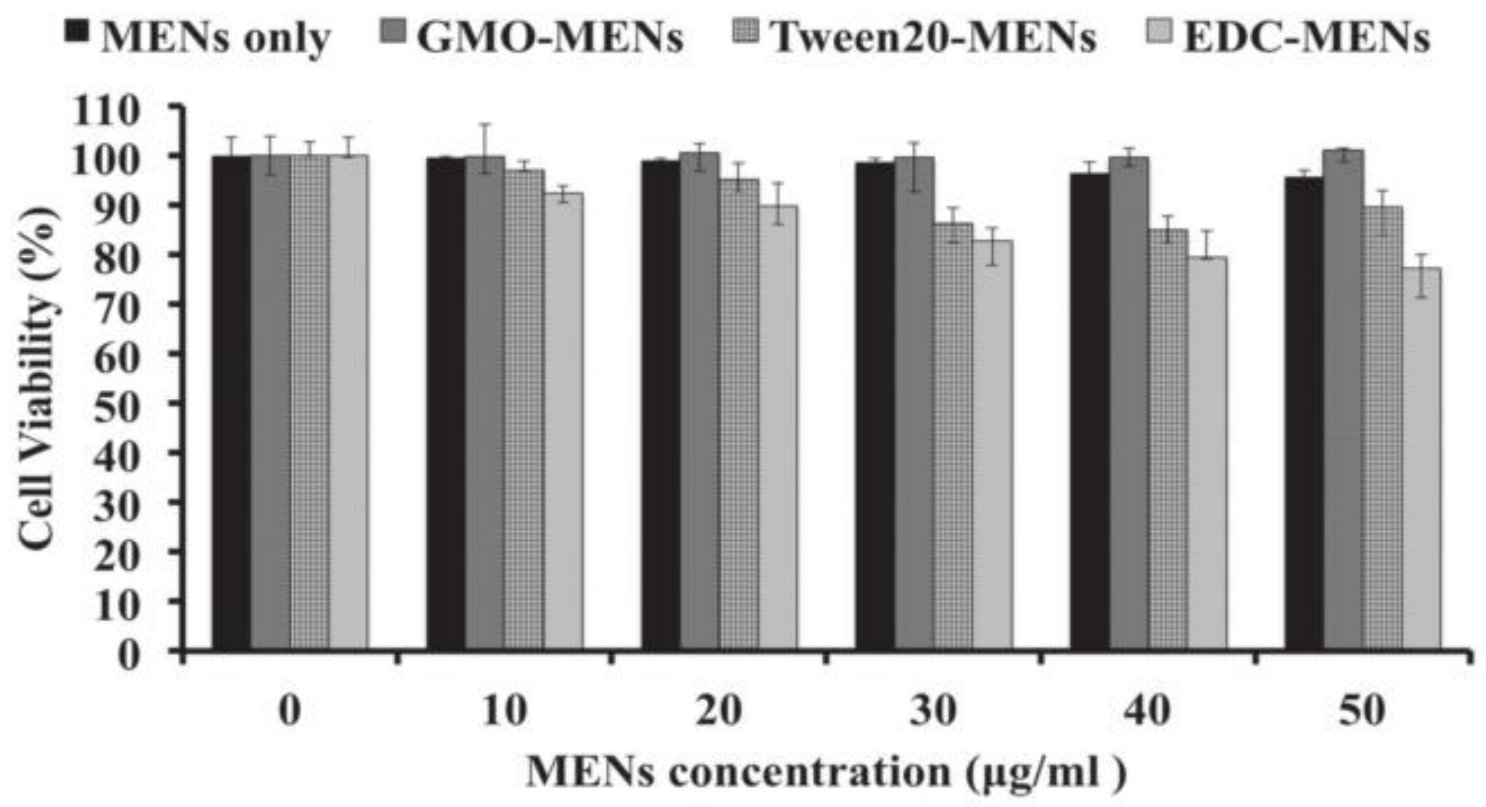

Figure 16. Functionalized MEN XTT cytotoxicity assay.

SKOV-3 cells are treated with different concentrations of the nanoformulations for 48 hours to determine any toxicity from the formulation alone.

The obvious benefit to using an externally applied magnetic field to trigger payload release from functionalized nanoformulations is the extent of options that are available for drug loading and release kinetics and the control that is achieved. For instance, releasing $100 \%$ of the drug PTX from GMO-coated MENs requires either a low, 10 Oe field at $1000-\mathrm{Hz}$ frequency or a d.c. $(0 \mathrm{~Hz})$ field of $100 \mathrm{Oe}$. This is especially useful in situations where a magnetic field gradient is desired to externally steer the nanoformulation to the target tissue, because the option to trigger payload release by simply increasing the frequency makes the treatment much more versatile.

Two important concepts must be considered when utilizing an intermediate functional layer: (a) the field required to break the bond between the nanoparticle and the 
intermediate layer must be greater than the bond required to break the bond between the intermediate layer and the payload; (b) the zeta potential, binding kinetics, and release kinetics are all directly related, i.e. nanoformulation with more negative zeta potentials will take up the payload less efficiently but will release it more readily under an external field, and vice versa - nanoformulations with higher zeta potentials will take up the payload more readily but will require a stronger field to release that payload. With these principles in mind, a MEN-based drug delivery system can be composed for virtually any drug delivery or imaging application.

\section{$\underline{3.5 \text { Conclusion }}$}

These three studies directly inspired this body of dissertation work. We wanted to continue with the next logical steps in testing our nanomedicine (i.e. in-vivo experiments), but also to address some of the critical feedback we received in response to these publications. For instance, the experiments detailed in Chapter 4 were developed to address the lack of direct confirmation that MENs have entered the cancer cells in the ovarian cancer in-vitro study, as this study only tracked the movement of the carried PTX payload. Furthermore, armed with the knowledge that MENs offer a physics-based, reliable, externally controlled, targeted drug delivery and release platform that has been confirmed experimentally in multiple in-vitro models, this dissertation project aimed to demonstrate the applicability of MENs as a viable cancer treatment in a murine model. 


\section{CHAPTER 4: MENS USE A PHYSICAL MECHANISM TO ENTER CANCER CELLS}

\section{$\underline{4.1 \text { Introduction }}$}

This chapter covers some of the fundamental components of the MEN platform. First, the MEN synthesis process is described, followed by the functionalization and drug-loading process, and characterizations of the platform. Additionally, this chapter shows an in-vitro experiment designed to prove that the physics based approach of this platform allows the nanoparticles to localize inside cancer cells. In this experiment, cells are incubated with the MEN nanoformulation in the presence of a d.c. magnetic field, a.c. magnetic field, or neither. After the incubation, the cells are fully washed of any leftover media. Then, the cells are lysed and the internal contents of the cells are analyzed for the presence of MENs. For MENs to be located in the cell lysate, they must have been internalized with the nanoelectroporation effect caused by the magnetic field inducing the magneto-electric coupling intrinsic to the MENs.

\subsection{Experimental Procedures}

\subsubsection{MENs Preparation}

$\mathrm{CoFe}_{2} \mathrm{O}_{4}$ nanoparticles were prepared by hydrothermal method:

Core Preparation: In $15 \mathrm{ml}$ of dionoized water, dissolve $0.058 \mathrm{~g}$ of $\mathrm{Co}\left(\mathrm{NO}_{3}\right)_{2} \cdot 6 \mathrm{H}_{2} \mathrm{O}$ and $0.16 \mathrm{~g}$ of $\mathrm{Fe}\left(\mathrm{NO}_{3}\right)_{3} \cdot 9 \mathrm{H}_{2} 0$. Heat solution to $120^{\circ} \mathrm{C}$ and add $0.2 \mathrm{~g}$ of polyvinylpyrrolidone dissolved in $5 \mathrm{ml}$ of aqueous $0.9 \mathrm{~g}$ of sodium borohydride. Maintain solution at $120{ }^{\circ} \mathrm{C}$ for 12 hours to precipitate $\mathrm{CoFe}_{2} \mathrm{O}_{4}$ core nanoparticles. 
Shell Preparation: $\mathrm{The}^{\mathrm{BaTiO}} 3$ precursor solution is prepared by dissolving $0.029 \mathrm{~g}$ of $\mathrm{BaCO}_{3}$ and $0.1 \mathrm{~g}$ of citric acid in $30 \mathrm{ml}$ of deionized water and by dissolving $0.048 \mathrm{ml}$ titanium isopropoxide and $1 \mathrm{~g}$ of citric acid in $30 \mathrm{ml}$ of ethanol. Both solutions are added to $0.1 \mathrm{~g}$ of dry $\mathrm{CoFe}_{2} \mathrm{O}_{4}$ core nanoparticles and the resulting solution is sonicated for at least two hours to fully disperse the cores in the shell precursor solution.

$\mathrm{CoFe}_{2} \mathrm{O}_{4}-\mathrm{BaTiO}_{3}$ core-shell MENs: The sonicated mixture is dried at $60{ }^{\circ} \mathrm{C}$ for 12 hours with continuous stirring at $200 \mathrm{rpm}$. The polymerized mixture is transferred to a highheat compatible ceramic dish. The mixture is subjected to calcination at $780{ }^{\circ} \mathrm{C}$ for 5 hours in a CMF1100 furnace and then cooled at $52{ }^{\circ} \mathrm{C} /$ min to yield $30 \mathrm{~nm}$ $\mathrm{CoFe}_{2} \mathrm{O}_{4}-\mathrm{BaTiO}_{3}$ core-shell MENs.

\subsubsection{MENs Functionalization}

A $2 \mathrm{~nm}$ thick coating of GMO is deposited onto the MENs. First, $0.1 \mathrm{mg}$ of GMO is incubated with $5 \mathrm{mg}$ of MENs in $5 \mathrm{ml}$ of PBS (pH 7.4) buffer for $12 \mathrm{~h}$. This solution is gently agitated on a spinner throughout the incubation to achieve uniform surface modification. After incubation, the solution is centrifuged at 20,000 rpm for $20 \mathrm{~min}$ at $10{ }^{\circ} \mathrm{C}$ and the supernatant discarded. To wash away any unbound GMO, the obtained pellet is resuspended in ethyl acetate:acetone (70:30) solution, agitated, and recentrifuged to discard the supernatant. This is done three times to ensure all unbound GMO is removed. Surface-modified MENs are lyophilized and stored at $4{ }^{\circ} \mathrm{C}$ until further use. 


\subsubsection{PTX Drug Loading}

PTX is non-covalently bonded to the GMO functional layer on the MENs. First, $50 \mathrm{mg}$ of PTX (dissolved in 70:30 methanol to PBS) is added to a solution of $100 \mathrm{ml}$ GMOcoated MENs ( $5 \mathrm{mg} / \mathrm{ml}$ concentration) suspended in $900 \mathrm{ml}$ of the PBS. This nanoparticle and drug mixture is incubated for 3 hours on a rotator for gentle agitation. Finally, the solution is centrifuged at $14,000 \mathrm{rpm}$ for 10 minutes at $10^{\circ} \mathrm{C}$, where any unbound PTX is discarded in the supernatant.

\subsubsection{TEM \& EDS}

A Phillips CM-200 200 kV Transmission Electron Microscope (TEM) with Energy Dispersive Spectroscopy (EDS) option was used to obtain TEM images and EDS profiles.

\subsubsection{Magnetic Field Dependent Zeta Potential}

The magnetic field dependence of the MENs' surface charge is measured using Malvern Zetasizer. To simulate the charge of MENs in the blood, $0.5 \mathrm{mg}$ of MENs are suspended in PBS (pH 7.3, similar to human blood $\mathrm{pH}$ ) inside a $1 \mathrm{ml}$ cuvette. The cuvette is then exposed to an electromagnet-based uniform magnetic field ranging from 0 to $+/-100 \mathrm{Oe}$ in 10 Oe increments. The polarity of the field did not play an effect.

\subsubsection{STS Measurements of I-V Curves}

The I-V curves are obtained using the scanning tunneling spectroscopy (STS) function of scanning tunneling microscopy (STM) mode of Multimode. The curves are from a direct point contact between the STM nanoprobe and a MEN in the influence of a 200 Oe d.c. 
magnetic field running along the central orientation. The tip distance is kept constant and the tunneling current (I) and voltage (V) are variable. The magnetic field is created by a custom multi-turn coil wrapped around the sample.

\subsubsection{Vibrating Sample Magnetometry}

A room-temperature Lakeshore vibrating sample magnetometer (VSM) with a 3-T magnetic field sweep was used to measure the key magnetic properties of nanoparticles under study. This includes the magnetization saturation and the magnetic coercivity.

\subsubsection{Fourier Transform Infrared Spectra}

FTIR absorption spectrum measurements are taken on the JASCO-4100 instrument. FTIR samples are prepared by placing one drop $(10 \mu \mathrm{l})$ of the desired nanoformulation at concentration $1 \mathrm{mg} / \mathrm{ml}$ on a pre-cleaned silicon wafer and drying the sample overnight.

\subsubsection{Spectrophotometry}

A Cary 100 Bio UV-visible spectrophotometer at $230 \mathrm{~nm}$ (unless otherwise noted) is used to calibrate and record all PTX measurements, as described in other literature [67].

\subsubsection{Cell Culture \& Lysis}

SKOV-3 cells purchased from American Type Culture Collection (Manassas, Virginia) were used in these experiments. Cells are maintained in McCoy's 5A medium (Life Technologies, New York) supplemented with 10\% fetal bovine serum (Sigma-Alrich) and $1 \%$ penicillin-streptomycin (ScienceCell). Cells are cultured in an incubator maintained at $37{ }^{\circ} \mathrm{C}$ temperature, $5 \% \mathrm{CO}_{2}$, and a humidified atmosphere. 
For the cell lysis experiment, cells are first seeded with a density of 0.7 to $1.0 \times 10^{6}$ cells in T-25 flasks and incubated overnight. Cells are allowed to reach full confluence, with the media being replaced every other day. At the beginning of each experiment, the media is discarded and replaced with $3 \mathrm{ml}$ of fresh media in which the tested nanoformulation is suspended, and incubated under a magnetic field. Figure 17 demonstrates the magnetic field incubation setup. The tested fields are no field, d.c. field, and d.c. + a.c. fields.

After the timed magnetic field treatment are complete, the flasks are removed from the incubator, the media discarded, and the cells washed. The cells are then trypsinized to detach from the flask, washed three times in chilled PBS, resuspended in $1 \mathrm{ml}$ of dimethyl sulfoxide (DMSO), and incubated for 1 hour at $37^{\circ} \mathrm{C}$. This way, all remnants of media with any leftover nanoformulation are removed before the cells are lysed with the DMSO. After the lysis incubation in complete, the solution is vortexed and centrifuged at 4,500 RPM for 10 minutes in order to pellet the cellular debris. The intracellular components remain in the supernatant.

The cell lysate is measured using spectrophotometry in scanning mode. A standard curve is generated by resuspending known concentrations of drug in DMSO and diluting it in 1 $\mathrm{ml}$ of PBS for reference curves so that each peak absorbance (at a specific wavelength) on the curve corresponds to a known concentration of drug. It is important to note that the amount of drug ultimately found in the cell lysate is highly dependent on the number of cells in the sample. The number of cells in the sample is therefore normalized by measuring the protein content of that sample. This is achieved by halting the protein 
degradation process immediately after the lysate is collected using a Bio-Rad Protein Assay Kit (Bradford method) followed by immediately measuring the absorbance in the $587-591 \mathrm{~nm}$ range.
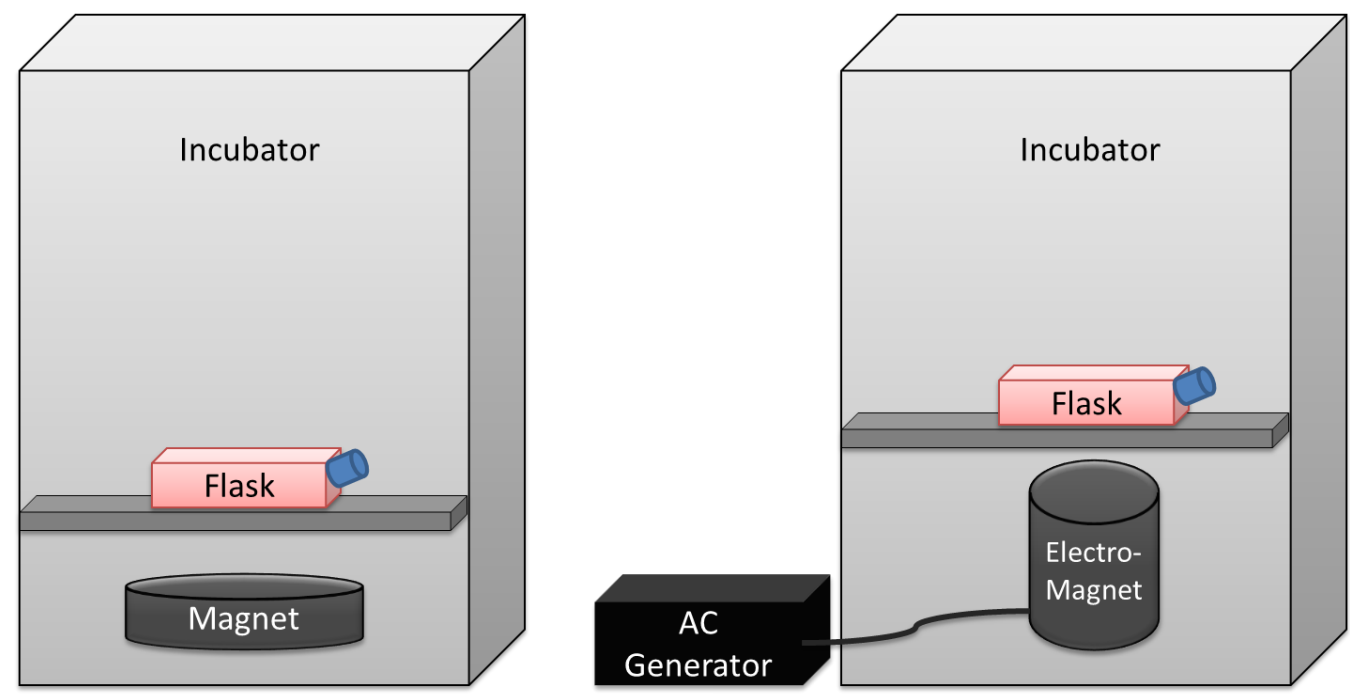

Figure 17. Cell culture with magnetic field setup.

A confluent flask of cancer cells is positioned directly above a magnet or electro-magnet inside the incubator to expose the cells to a magnetic field while maintaining ideal incubation parameters $\left(37^{\circ} \mathrm{C}\right.$ temperature, $5 \% \mathrm{CO}_{2}$, and a humidified atmosphere).

\subsubsection{Scanning Probe Microscopy}

A scanning probe microscope (SPM) Multimode was used to collect atomic force microscopy (AFM) and magnetic force microscopy (MFM) images of the nanoformulations in the different experimental scenarios. The images are collected with a CoCr-based "hard" magnetic nanoprobe in a lift mode at a scan height of $10 \mathrm{~nm}$. In this scanning mode, each image line is scanned two times; the first scan measures the topography through the Van der Waals interaction (data type: height, $\mathrm{Z}$ range: $75 \mathrm{~nm}$ ) and the second scan measures the magnetic signal through the magnetic force by lifting the probe at the scan height distance (data type: phase, $\mathrm{Z}$ range: 500 ). The magnetic 
coercivity and the saturation magnetization of the MFM probe are believed to be on the order of $500 \mathrm{Oe}$ and $500 \mathrm{emu} / \mathrm{cc}$, respectively.

\subsection{Results}

\subsubsection{The Characterization of the Physical Features of MENs}

Figure 18 summarizes the unique and defining physical features of MENs. The hydrothermal synthesis method described in the procedures section yields a core-shell structure of MENs that is clearly visible under TEM (Figure 18a); the square core without the surrounding perovskite shell would represent $\mathrm{CoFe}_{2} \mathrm{O}_{4}$ ferromagnetic nanoparticles (FNs). The VSM hysteresis loops for MENs and FNs of identical sizes (30 nm) demonstrates why FNs are utilized as a control for the effects of magnetization alone (Figure 18b): FNs possess approximately 40 times the saturation magnetization of MENs ( 1 emu/g for MENs versus the $\sim 401 \mathrm{emu} / \mathrm{g}$ for FNs), but no magneto-electric effects. The zeta potential of MENs is highly dependent on the strength magnetic field exposure, but not on the orientation of the applied field (Figure 18c). Since zeta potential characterizes the surface charge equilibrium from the double-layer chemistry of the particles in a liquid solution, this charge is an important consideration for cellular uptake, as electrically charged cell membranes would be interacting with this charge. In the case of MENs, the transition from no field to a field of $100 \mathrm{Oe}$ (positive or negative direction not affecting results) increased the zeta potential by over $30 \%$. This is an important component of the externally triggered payload delivery mechanism of the MEN drug delivery system, as the MENs will not readily interface with the electrically charged membranes of cells until a magnetic field is applied and the MENs' zeta potential 
increases. The effect an applied magnetic field has on MENs can be visualized with MFM imaging (Figure 18d). Here, $30 \mathrm{~nm}$ MENs are deposited onto a silicone oxide substrate and magnetized with an external field of 100 Oe. The direction of the field can then be seen in the image as the dipoles of the MENs are oriented directly along the field direction, transitioning from dark to light in the direction of the field. The conductivity of the MENs is shown in the STS I-V curve measurement plot (Figure 18e). A magnetic field of 100 Oe in either direction significantly increases the effective conductivity of the MENs.
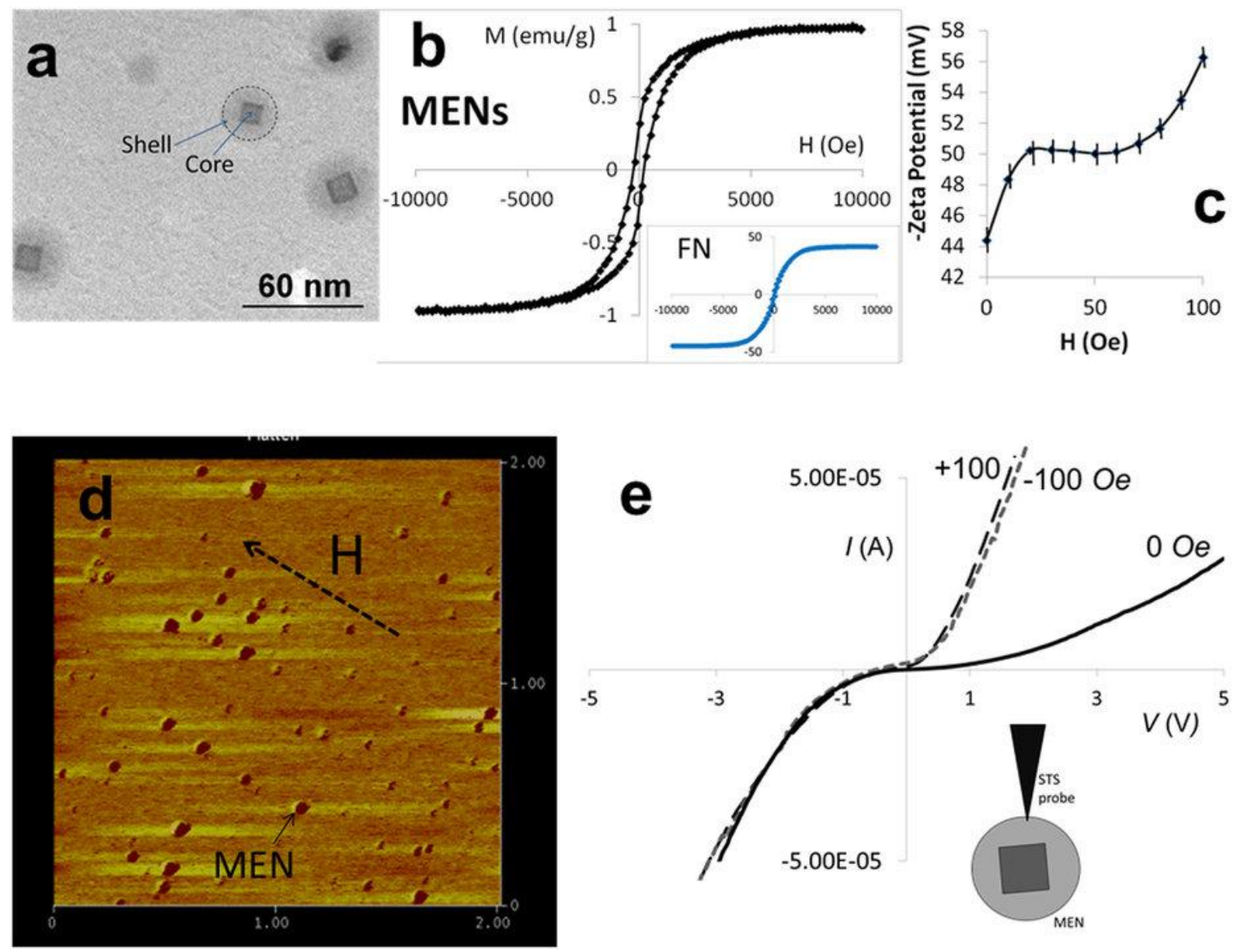

Figure 18. Physical characterizations of MENs.

(a) TEM image showing the coreshell nanostructure of MENs with the spinel $\mathrm{CoFe}_{2} \mathrm{O}_{4}$ core and the perovskite $\mathrm{BaTiO}_{3}$ shell. (b) M-H hysteresis loop of MENs, with the loop for FNs shown in the insert for comparison. (c) Magnetic field dependence of zeta potential for MENs in PBS buffer. (d) MFM image showing the dipole nature of $30 \mathrm{~nm}$ MENs where $\mathrm{H}$ is the direction of the magnetic field. (e) STS I-V curve 
measured from a point contact between the tungsten nanoprobe of a STM setup and a MEN at three different field values $(-100,0$, and $100 \mathrm{Oe})$, with the magnetic field applied along the central axis.

These physical properties make MENs uniquely suited as nanocarriers to safely transport their payload through circulation while maintaining a lack of bioavailability until cellular internalization and payload release is triggered with an externally applied magnetic field on demand.

\subsubsection{The Cellular Internalization of MENs \& Their Payload}

We used SPM imaging to demonstrate that MENs enter the cancer cells and spectrophotometry to demonstrate that the payload they carry enters with them. Flasks of cancer cells are treated with a MENs-PTX nanoformulation and exposed to different magnetic fields. The cells are fully washed of external media prior to being burst open to investigate the internal contents. SPM, and specifically the MFM mode of SPM, is selected to identify internalized MENs because their dipole nature makes them unmistakably recognizable in solution. Figure 19 shows a MENs only solution imaged as a baseline. All cellular contents lack the dark to light transition distinctive to the MENs. The carried payload concentration is calculated more specifically using spectrophotometry; the calibration parameters used are shown in Figure 20. 


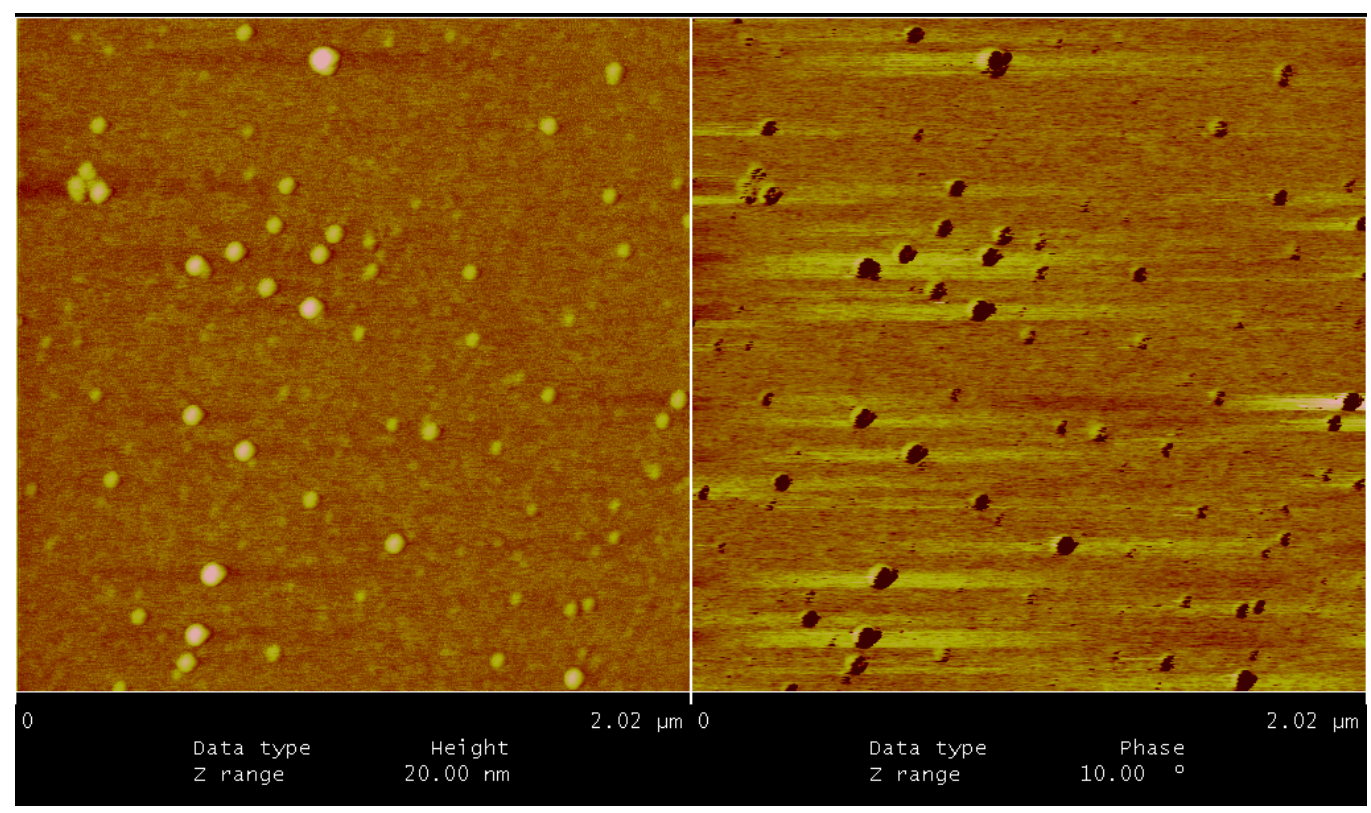

Figure 19. SPM Image of MENs only.

The left side shows a height reading while the right side shows a phase reading of the same scan of MENs without any cellular contents.
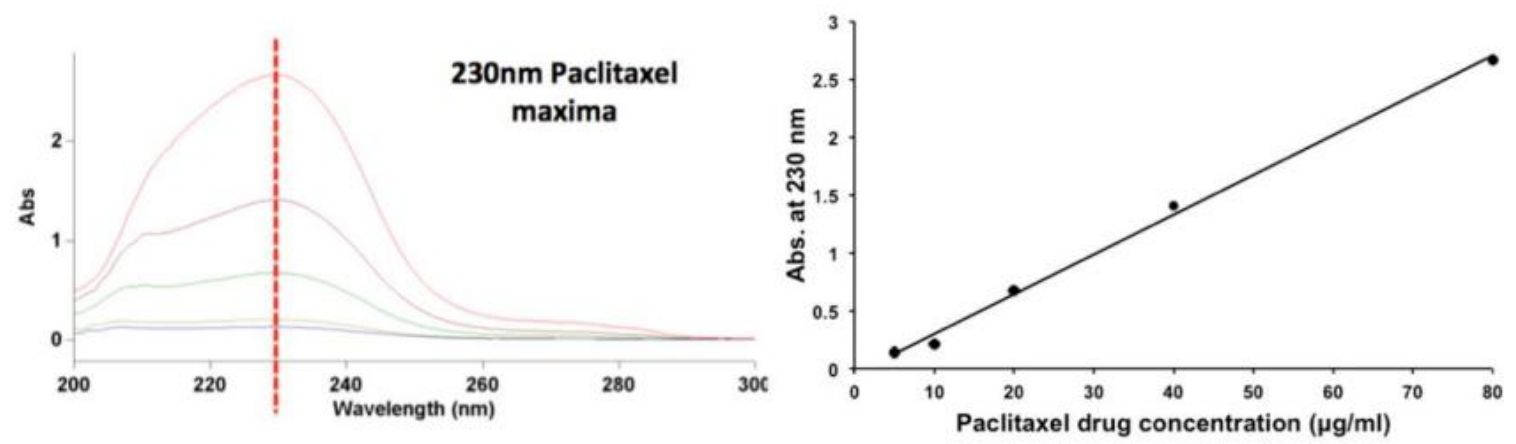

Figure 20. PTX calibration for spectrophotometer

(a) PTX absorption maxima at a wavelength of $230 \mathrm{~nm}$. (b) Standard linear calibration curve for PTX created for different concentrations of the drug.

Figure 21 shows the field dependent nature of the MENs' cellular internalization.

When the cells are incubated with the nanoformulation and no magnetic field, the nanoformulation cannot be detected within the cell lysate. However, a d.c. magnetic field of 100 Oe applied to the flask during the nanoformulation incubation time yielded cell 
lysate full of the MENs. These results support that the nanoelectroporation effect described earlier relies on a magnetic field application to manifest.
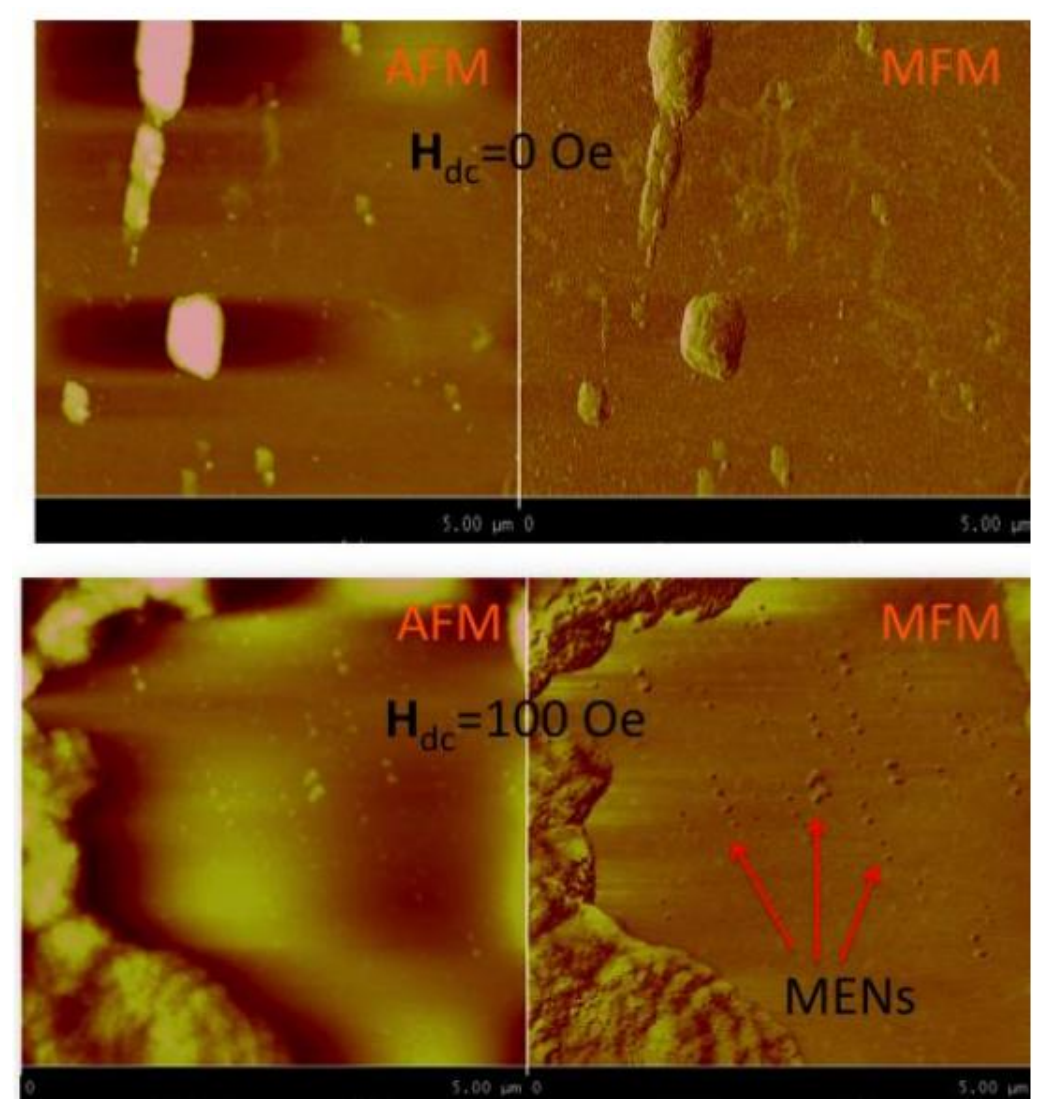

Figure 21. SPM showing MENs only in field exposed cells.

AFM and MFM processing of the same scan show that no MENs are present intracellularly in the absence of a field (top) but are clearly present post exposure to a 100 Oe d.c. field (bottom).

Figure 22 shows that the carried payload, in this case PTX, was also internalized on the MEN nanocarrier. Very little bioavailable PTX can be detected in the cell lysate when only a d.c. magnetic field is applied. However, this is because the drug is still bound to the MENs under the d.c. field needed to internalize the nanoformulation. The PTX can be detected in the cell lysate after the application of an a.c. magnetic field of Oe 50 at $100 \mathrm{~Hz}$. The cell lysate only then shows peaks at similar wavelengths as the calibrated PTX, indicating that there is free drug present in the cell lysate. 


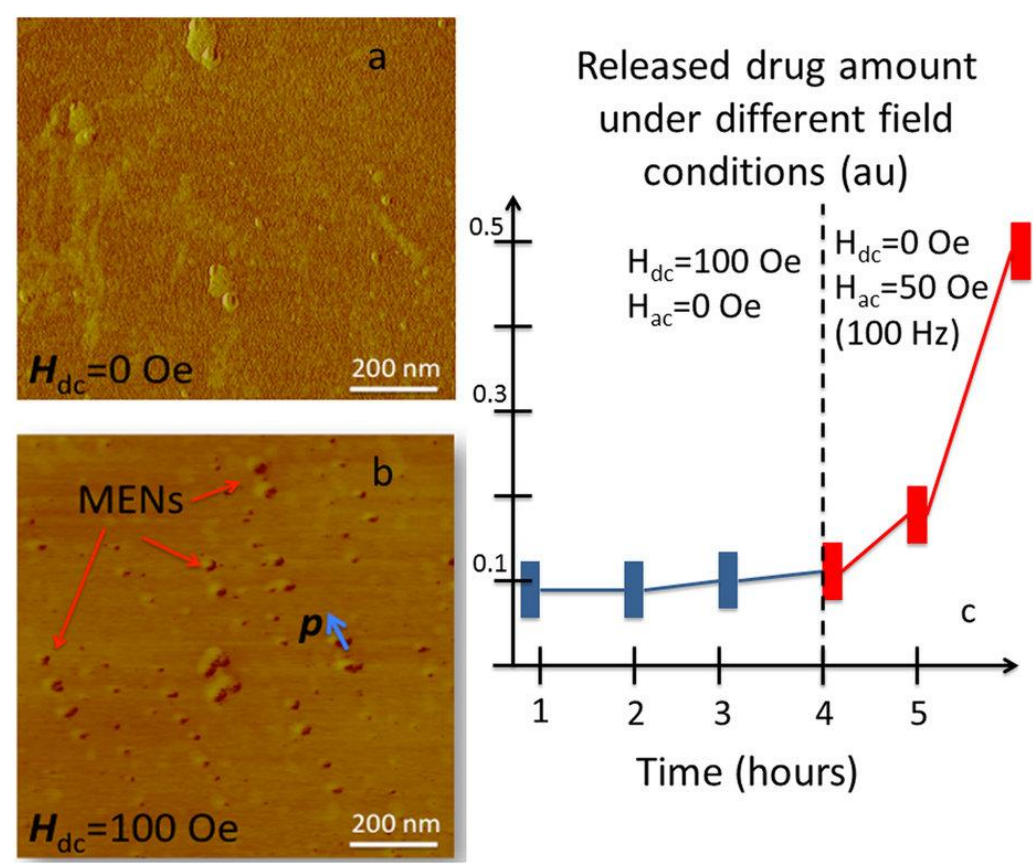

Figure 22. Field dependence of intracellular contents of treated cells.

The left side of the figure shows the field dependence of MENs internalization with MFM images. The right shows the field dependence of the payload drug concentration intracellularly. (a) Prior to exposure, there are no magnetic signals in the cell contents. (b) After a d.c. field $\left(\mathrm{H}_{\mathrm{dc}}=100 \mathrm{Oe}\right)$ application, the dipole nature of MENs (blue arrow show orientation of field) reveals them to be inside the cells (red arrows). (c) Spectrophotometrically measured bioactive drug amount inside the cells normalized for cell protein content under different a.c./d.c.-field conditions over time (shown in arbitrary absorbance units).

\subsection{Conclusion}

This chapter focused on detailing some of the defining physical features of MENs that make them a strong drug delivery system, especially in the case of cancer treatment. As seen in Figure 18, MENs posses both a dipole from the magneto-electric effect and an electric charge from the negative (non-zero) zeta potential. Both of these physical attributes generate electric fields which grow with the strength of the external magnetic field that is used to generate them. The electric field generated by the MENs' electric charge $\left(\sim 1 / \mathrm{r}^{2}\right)$ is stronger than the electric field generated from the dipole $\left(\sim 1 / \mathrm{r}^{3}\right)$. This unique generation of electric fields by the MENs is exploited here for cancer discrimination and for loaded drug release. 
The aim of the cell lysate experiment was to illustrate that the MEN nanoformulation internalizes itself into the cell, along with the carried payload. This experiment proved that both the MENs and the payload they carry are taken up by the cells in the presence of a weak magnetic field. The publication described in Chapter 3.3 has already shown that MENs facilitate the entry of PTX into cancer cells, with high preference over healthy cells, in the presence of a magnetic field. However, that study tracked florescent PTX rather than the MENs themselves. Now it has been confirmed that MENs do internalize into cancer cells, as they were located using MFM inside the lysate of treated cells. Furthermore, the cell lysate experiments showed that the carried treatment is not made significantly bioavailable even inside the cell until deliberately released with an a.c. magnetic field (or a stronger d.c. magnetic field). This attribute will ensure that only negligible amounts of the drug are lost prematurely off the nanocarrier until essentially all of the nanoformulation has been internalized by the cancer cells. It is also important to note that there is little cellular internalization of the nanoformulation until a magnetic field is administered over the MENs. The zeta potential and conductivity of MENs likely has a role in this field-induced nanoelectroporation, as an applied field is converted into an electric output as well as an increased zeta potential by the MENs.

It is also important to note that there was no functionalization performed on the MENs to make them unique to the type of cancer used in this experiment to demonstrate the internalization process. The mechanism of cellular entry is entirely physical in nature. The physics-based approach used by the MENs to gain access to the internal portion of cancer cells is highly advantageous from a diversification standpoint, as practically any type of cancer can be treated using nearly identical formulations. Other types of 
nanoformulations or other drug delivery platforms rely on targeting molecules, such as antibodies, to draw the treatment to the cancer cells. The magneto-electric coupling of MENs pulls the MENs directly towards the cellular membrane of cancer cells, preferentially due to the differing electric cell membrane properties, when that coupling is induced by an applied magnetic field. Furthermore, additional targeting molecules can also be loaded onto the MENs as needed to further tailor and strengthen the individual treatment platform.

The separation of the functions of nanoformulation internalization (using a weak d.c. magnetic field) and payload release (using a stronger or a.c. field) allows for three discreet steps in the drug delivery treatment process to be performed as needed: (1) initial administration, (2) targeting assistance/guidance, and (3) payload release. Since these steps are externally controllable, the optimal amount of time can be waited before proceeding from one step to the next. This allows for an unprecedented amount of control in the treatment process.

Together with the publications described in Chapter 3, these experiments have established the exact trajectory of the nanoformulation when used to treat cancer cells under different magnetic fields. The MENs platform reliably delivers PTX to ovarian cancer cells in-vitro. This body of evidence supports that this nanomedicine platform will work to successfully treat cancer in an in-vivo model, and it resulted in IACUC approval for the animal studies presented in the next chapter. 


\section{CHAPTER 5: MENS EFFECTIVELY CURE CANCER IN A MOUSE MODEL}

\section{$\underline{5.1 \text { Introduction }}$}

This chapter describes the first instance of MENs curing cancer in an animal model. We continued working with SKOV-3 human ovarian carcinoma cells in order to maintain consistency with the previous studies described in Chapters 3.3, 3.4, and 4, and to establish that this particularly aggressive cancer type can be successfully treated with a MEN-based nanoformulation in-vivo. We established a humanized mouse model of human ovarian cancer by implanting SKOV-3 carcinoma into immunocompromised mice. Once a visible tumor has formed, a weekly PTX-nanoformulation treatment starts and continues until an endpoint is reached (i.e., the tumor grows too large or the cancer has been cured). In addition to the main goal of demonstrating that our MEN drug delivery platform can cure cancer in the mouse model, we investigated four additional aspects of the treatment for optimization of the treatment:

1. We compare two nanoformulation delivery methods: localized injection and systemic injection. A localized injection, where the treatment is injected in very close proximity to the tumor and flushes the tumor in the medicine, versus a systemic injection, where the treatment is injected into a vein and carried through the bloodstream, offer different benefits. A localized injection offers immediate access to the treatment without any travel through the periphery, however only the peripheral cells of the tumor get the maximum contact. A systemic injection will have to travel through the entirety of the vasculature until it reaches the tumor, however, every cell inside the tumor maintained by perfusion will ultimately be 
exposed to the treatment.

2. We compare the MEN cancer treatment platform to two other nanoparticle drug delivery platforms: MN and PLGA nanoparticle based platforms. MNs control for the magneto-electric coupling that is unique to MENs, as MNs are highly magnetic (in fact, MNs have stronger magnetic properties compared to MENs) but have no electric properties. PLGA nanoparticles, a fully organic particle, represent an FDA approved nanoparticle cancer treatment platform that controls for the magnetic aspects of both MENs and MNs [68]. It also allows us to compare our new treatment to a treatment currently on the market and being utilized in modern medicine.

3. Peripheral targeting molecules in the form of Human Epidermal Growth Factor Receptor 2 (HER-2) antibodies and sphingosylphosphorylcholine (SPC) ligands are tested on the nanoformulations to determine if they improve the cancer targeting capabilities of the nanoformulation. Both of these molecules are over expressed on the surface of human ovarian cancer cells, making them good candidates for active targeting molecules. HER-2 [69] was extensively investigated in our previous in-vitro study [60], and SPC was selected as an alternative targeting molecule candidate [70].

4. Different sizes of MENs are tested as the nanocarriers for PTX. All prior experiments (Chapters 3 and 4) were performed with $30 \mathrm{~nm}$ sized MENs. More recently, we developed procedures for synthesizing MENs of different size ranges. Different sized particles offer unique combinations of surface area and magnetic and electric properties. 
Furthermore, the previously described effects of d.c. and a.c. magnetic fields are investigated in the context of an in-vivo model.

\section{$\underline{5.2 \text { Experimental Procedures }}$}

\subsubsection{MEN \& MN Preparation and Drug Loading}

MENs are synthesized using the hydrothermal method, coated with a $2 \mathrm{~nm}$ layer of GMO, and loaded with PTX as described in Chapter 4.2.

The MNs used are the same component used in the ferromagnetic core component of the MENs, i.e. MENs sans their perovskite shell. Therefore, MNs preparation consisted of the cobalt ferrite precipitation protocol follow immediately by GMO coating and PTX loading. The properties of the base FN cores are shown in Chapter 4.3.

To control the size of the nanoparticles synthesized, the heat parameters of the furnace are adjusted. By adjusting the temperature and timing of the warm up and cool down phase, we were able to synthesize larger nanoparticles $(\sim 100 \mathrm{~nm})$ and smaller nanoparticles $(\sim 10 \mathrm{~nm})$ than our original MENs nanoformulation $(\sim 30 \mathrm{~nm})$.

\subsubsection{PLGA Nanoparticle Preparation}

PLGA nanoparticles are synthesized by the emulsion solvent evaporation method [71]. An important distinction is that this is an organic nanoparticle that encapsulates the payload rather than carrying it on the surface like inorganic nanoparticles tend to. Thus, the PTX is incorporated directly into the formulation of the particle. Briefly, $10 \mathrm{ml}$ of the organic phase (consisting of $100 \mathrm{mg}$ of PLGA, MW 5000, $5 \mathrm{mg}$ of PTX, and $50 \mu \mathrm{l}$ of 
triethyl-amine in $10 \mathrm{ml}$ of dicloromethane) is added to $20 \mathrm{ml}$ of aqueous the phase (3\% PVA). The saturated organic and aqueous phases are emulsified in a sonicator for 10 minutes. The formed PLGA nanoparticles are separated from the solution via centrifugation of the evaporated solvent at 20,000 rpm. The resulting nanoparticles are resuspended in $2 \mathrm{ml}$ of PBS (pH 7.4) for further use. PLGA particles are used as is, and are not treated with GMO or any other functional layer.

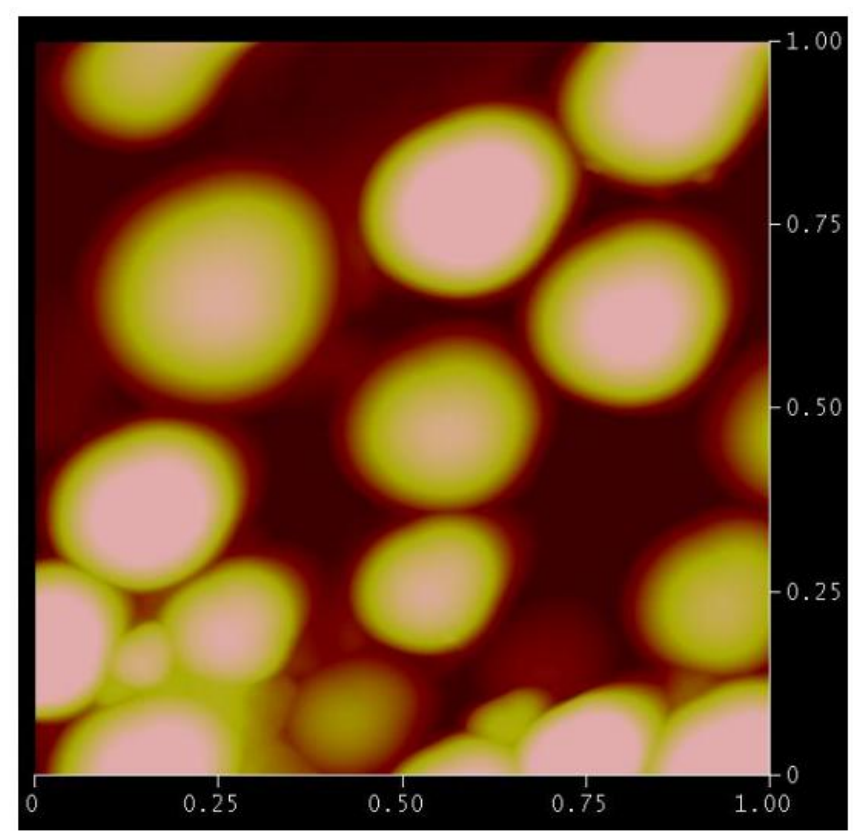

Figure 23. PLGA nanoparticle imaged with AFM.

These organic polymer nanoparticles encapsulating PTX are approximately $200 \mathrm{~nm}$ in size.

\subsubsection{Antibody \& Ligand Tagging}

The procedure for affixing the HER-2 antibodies and SPC ligands to the MENs, MNs, and PLGA particles was adapted from Kockbek et al. [72]. In the case of MENs and MNs, where both the targeting molecule and PTX are carried on the surface, these steps were performed after GMO functionalization and prior to the PTX drug loading (in the case of PLGA particles, where the PTX is encapsulated during the synthesis of the 
particle, these steps were prepared last). This technique of layering the targeting molecule underneath the PTX payload for the inorganic nanoparticles was quantified and confirmed in our previous study [60].

Briefly, $25 \mu \mathrm{l}$ of N-(3-Dimethylaminopropyl)-N'-ethyl-carbodiimide hydrochloride (EDC) and $25 \mu \mathrm{l}$ of N-hydroxysuccinimide (NHS), both at $1 \mathrm{mg} / \mathrm{ml}$ concentration in PBS, were added to $1 \mathrm{mg}$ of the nanoformulation is suspended in $500 \mu \mathrm{l}$ of PBS. The solution was allowed to incubate for four hours at room temperature with gentle stirring. After the incubation, the solution was centrifuged at $14,000 \mathrm{rpm}$ for 10 minutes in a centrifuge maintained at $10{ }^{\circ} \mathrm{C}$. The resulting pellet was washed three times with $1 \mathrm{ml}$ of the PBS to remove any unbound EDC and NHS. Then, the pellet was resuspended in 300 $\mu \mathrm{PBS}$ and $10 \mu \mathrm{l}$ of the antibodies or ligand $(1 \mathrm{mg} / \mathrm{ml})$ are added. The solution was incubated overnight at $4{ }^{\circ} \mathrm{C}$, with gentle stirring during the first two hours of incubation. The final solution was centrifuged at $14,000 \mathrm{rpm}$ for 10 minutes at $10{ }^{\circ} \mathrm{C}$ and the pellet was washed thrice with $1 \mathrm{ml}$ of the PBS.

\subsubsection{General Animal Care}

The mice used are of the SCID and Nude varieties, both purchased from Taconic. Mice were maintained under in the institutional animal care facility under a specific pathogenfree environment. The mice were housed in a climate control and day/night light cycle control maintained room. The veterinarian-approved guide for best animal practices in cancer research was followed [73]. Mice were kept in groups of 2-5 (larger groups preferred, with smaller groups used for uneven numbers) in solid floor caging with bedding, and provided with a plastic igloo shelter, enrichment nestlets, and free access to 
commercial mouse chow (global diet by Envigo) and water. Mice were carefully observed for general good health and well-being, as is characterized by well-groomed fur, clear eyes, strong appetite, enthusiastic nest-building, curiosity/investigative behavior, and other normal behaviors and physical traits. The weight of the mice was also monitored for any changes. Mouse identification was maintained with tail markings that were regularly reapplied as needed.

\subsubsection{SKOV-3 Xenograft}

SKOV-3 cells purchased from American Type Culture Collection (Manassas, Virginia) were used in these experiments. Cells are cultured in an incubator maintained at $37{ }^{\circ} \mathrm{C}$ temperature, $5 \% \mathrm{CO}_{2}$, and a humidified atmosphere and maintained in McCoy's 5A medium (Life Technologies, New York) supplemented with 10\% fetal bovine serum (Sigma-Alrich) and 1\% penicillin-streptomycin (Science-Cell). Once confluent, the cells were washed once with PBS buffer, detached by means of trypsinazation for 2 minutes, neutralized using trypsin neutralizing solution, and then centrifuged at $1500 \mathrm{rpm}$ for 5

minutes to precipitate the floating cells. The precipitated cells were resuspended in $100 \mu \mathrm{l}$ fresh McCoy's 5A medium; the density of the solution was $\sim 1 \times 10^{7}$ cells. This solution was injected into the right, outer back thigh of the mouse. The injection was performed on 8 week old mice under isoflurane anesthesia.

\subsubsection{Mouse Treatment}

No treatment was started until a tumor developed to a size of approximately $200 \mathrm{~mm}^{3}$, which could take up to 6 months. Therefore, treatment started on a rolling basis depending on when each mouse developed a large enough tumor to begin treatment. The 
tumor size was measured by a digital slide caliper and the tumor volume was calculated according to the following formula: volume $=$ width $^{2} \times$ length $/ 2$.

Each mouse was randomly assigned a treatment group. Once a tumor of at least $200 \mathrm{~mm}^{3}$ developed, treatment was administered weekly until an endpoint for the mouse had been reached. The defined end points were: a) the mouse maintained a lack of visible or palpable tumor; b) the tumor exceeded $2000 \mathrm{~mm}^{3}$; c) the tumor exhibited breakage or necrosis; d) the mouse appeared to be in distress or poor health.

The weekly treatments consisted of a fixed amount of PTX $(15 \mu \mathrm{g})$ carried by the appropriate amount of the nanoformulation being tested. Therefore, the amount of nanoparticles used varied between the nanocarrier types to adjust for different binding efficiencies/encapsulation rates and ensure that an identical amount of the payload makes up the treatment. Each test group had a positive control trial (no treatment) and additional controls consised of free-PTX (no nanocarrier, simply suspended in PBS) and non-drugloaded nanocarriers. Each treatment formulation was suspended in $20 \mu \mathrm{l}$ for injection.

The treatments were injected either subcutaneously or by intra venous (IV) injection. Both methods utilized isoflurane anesthesia and 39 gauge hypodermic needles. Subcutaneous injection was performed in the immediate vicinity of the tumor, but with no contact being made with the tumor mass. IV injection was performed on the lateral tail vein (alternating sides every week to allow time for healing). Mice were kept warm under anesthesia using an infrared (IR) bulb heat lamp, which also helped dilate the lateral tail vein for IV injection. 


\subsubsection{Magnetic Field Setup}

To safely establish the d.c. magnetic fields required to influence the inorganic nanoformulations in close vicinity to the tumor site, a small, high magnetic moment neodymium magnetic coin was affixed on top of the tumor site using $3 \mathrm{M}^{\mathrm{TM}}$ Vetbond ${ }^{\mathrm{TM}}$ adhesive. The coin was shed without any assistance in approximately one hour and was collected from the cage. This neodinium magnet is defined by a magnetic anisotropy normal to the plane, a saturation magnetization of $800 \mathrm{emu} / \mathrm{cc}$, and the generation of a d.c. field on the order of 100 Oe a few millimeters away from the surface. Therefore, the effect of the magnet was equivalent to application of a local 100-Oe external d.c. magnetic field immediately after each weekly administration of PTX-loaded MENs. Figure 24 shows a mouse with the Vetbond attached magnetic coin.

An a.c. magnetic field was established using a custom made cage setup shown in Figure 25. Here, an a.c. field of $50 \mathrm{Oe}$ at $100 \mathrm{~Hz}$ was generated immediately under the floor of a cage where a set of 4 electromagnetic coils was connected to two generators. Twentyfour hours after the mouse had been treated with the nanoformulation, it is placed in a different cage that is then placed on this setup for the duration of a.c. field exposure period of 1 hour. 

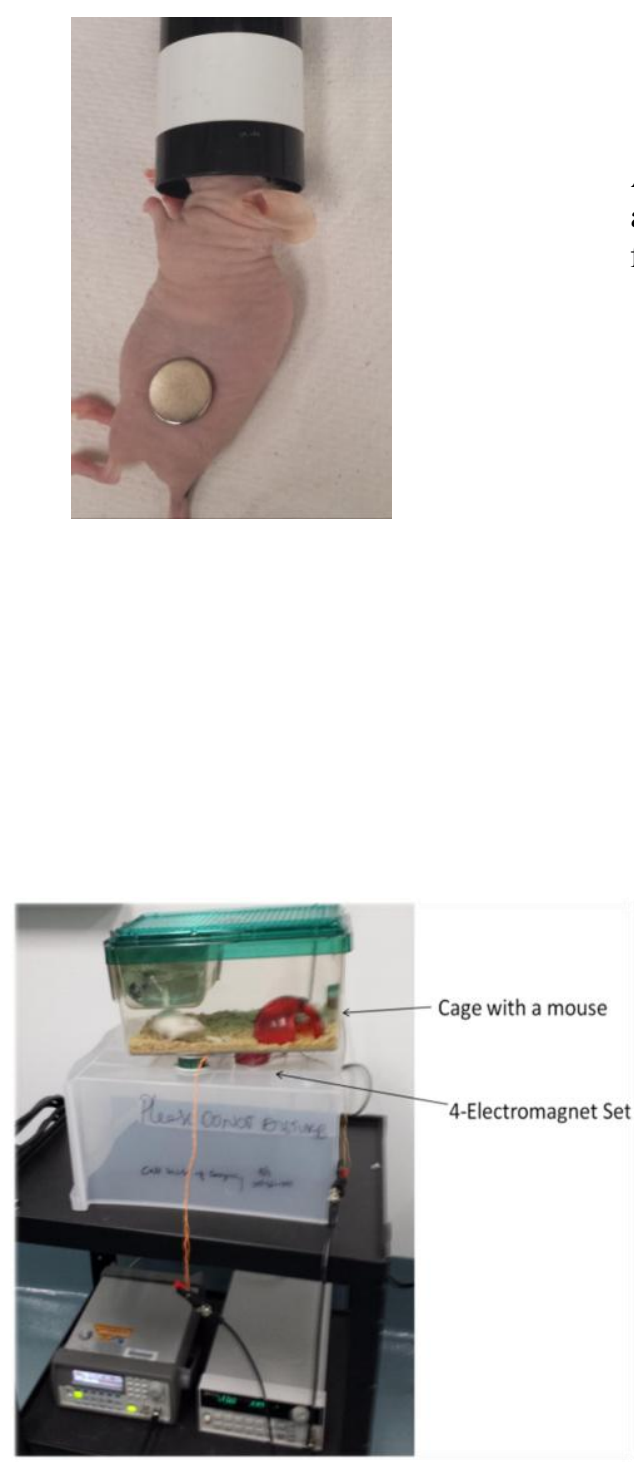

Figure 24. In-vivo d.c. field setup.

A d.c. field is generated directly at the site of the tumor by affixing a magnetic disk using Vetbond, a veterinary grade fixative.
Figure 25. In-vivo a.c. field setup.

An a.c. field is generated directly under the floor of a cage to create a safe and comfortable environment for the mouse for the duration of the a.c. exposure portion of treatment. 


\subsubsection{Tissue Preparation \& Imaging}

When an endpoint had been reached, the mice were euthanized by means of $\mathrm{CO}_{2}$ inhalation followed by cervical dislocation. The tissues of interest (tumor, liver, lungs, kidneys, and spleen) were excised and stored in a $10 \%$ formalin solution overnight at room temperature.

The tissues were processed using a VIP E 300 Tissue Tek Tissue processor SN 48940652 to create fixed, dehydrated, and cleaned cassettes. The tissues were transferred to warm paraffin filled molds using warm forceps and allowed to cool until the paraffin was solid. A Leica 2125 microtome as then used to cut 4- $\mu \mathrm{m}$ sections, which were flattened by floating in a $40{ }^{\circ} \mathrm{C}$ water bath before being placed on VWR $®$ Superfrost ${ }^{\circledR}$ Plus microscope slides to dry.

For optical imaging, cover-slips were added using Tissue Tek SCA Automated Coverslipper at this point, or after hematoxylin and eosin (H\&E) staining using standard procedure, e.g. using the MMI H\&E Staining Kit Plus. The sectioned tissue slides were imaged using Leica light microscope at 40X magnification.

\subsubsection{Infrared Imaging}

IR imaging was performed on both live animals for in-vivo tumor progression monitoring and for immunostaining of sectioned tissues to confirm the presence of the SKOV-3 cells. We used the HER2Sense 645 system from PerkinElmer. This fluorescent agent has an excitation and emission maxima of 643 and $661 \mathrm{~nm}$, respectively, and is safe for live 
animal use. Each dose of the agent was supplied with a solution of $100 \mu \mathrm{L}$ of $0.02 \mathrm{M}$ histidine, $0.02 \mathrm{M} \mathrm{NaCl}, 5 \%$ sucrose $(\mathrm{pH} 6.0)$.

For live mouse monitoring, the HER2Sense was administrated through IV injections in the lateral tail vein approximately 10 hours before an imaging session to maximize the imaging signal. For tissue immunostaining, the slides (prepared identically to those for H\&E staining) were incubated with the HER2Sense solution for 15 minutes at room temperature. Following incubation, the tissues were washed three times using PBS and mounted with ProLong-Gold anti-fade reagent. A Leica fluorescent microscope with a Cy5 filter at 40X magnification was used for the imaging.

\section{$\underline{5.3 \text { Results }}$}

\subsubsection{Localized Nanoformulation Delivery}

The subcutaneous trials were the first trials performed due to the simplicity of the treatment. Mice were treated once a week with their assigned formulation, and a magnet was affixed immediately after the peritumoral injection. Since the magnet naturally sheds in approximately one hour, the magnet was reaffixed daily to re-magnetize the nanoformulations for the duration of the treatment.

As shown in Figure 26, positive controls were established in the form of (a) no treatment; (b) initial treatment that is then halted (three treatments are administered before cessation); (c) MENs without any attached PTX; (d) free PTX without any nanocarrier. We saw a notable reduction in tumor growth rate when mice were treated with the GMO-coated MEN nanocarrier loaded with the PTX payload. In this trial, four 
mice were treated with an identical nanoformulation. Control mice that receive no treatment reached an endpoint in fewer than 10 days, and mice that received free-drug or only the nanocarrier reached an endpoint in just over 20 days. By direct comparison, as shown in Figure 27, all the mice undergoing the MENs-PTX treatment were doing well and maintaining controllable tumors between $\sim 100 \mathrm{~mm}^{3}$ and $\sim 700 \mathrm{~mm}^{3}$ at day 20 in their treatment. The entire progression of the MENs-PTX treatment is shown in Figure 28. All the mice that underwent MENs-PTX treatment performed much better than control mice, and were able to maintain a treatable level of the cancer for up to twice as long as control mice before reaching an endpoint. However, none of the mice were able to be fully cured using a peritumoral subcutaneous injection delivery method for the nanoformulation. For this reason, all further experiments were performed using the IV delivery approach.

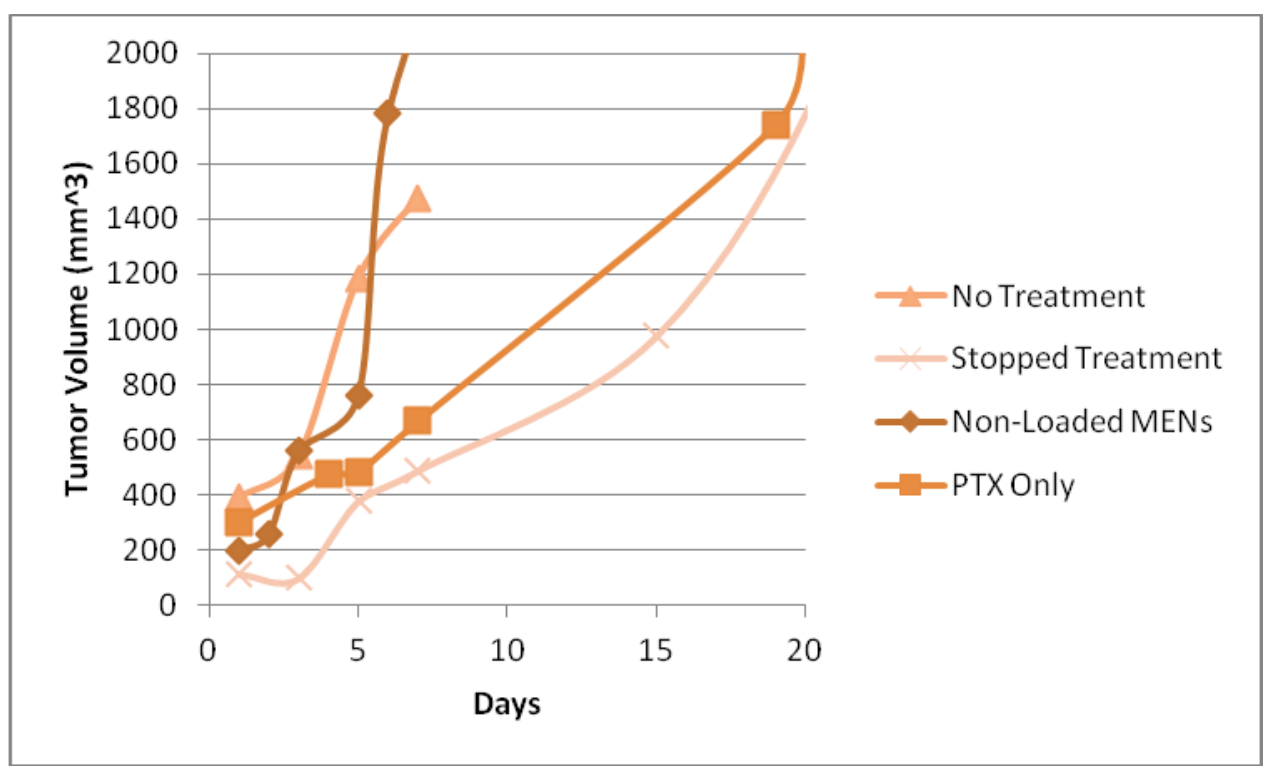

Figure 26. Positive controls.

References for the efficacy of the nanoformulation tested were first established by observing mice with no treatment, halted treatment, nanocarrier not loaded with the drug, and free drug. 


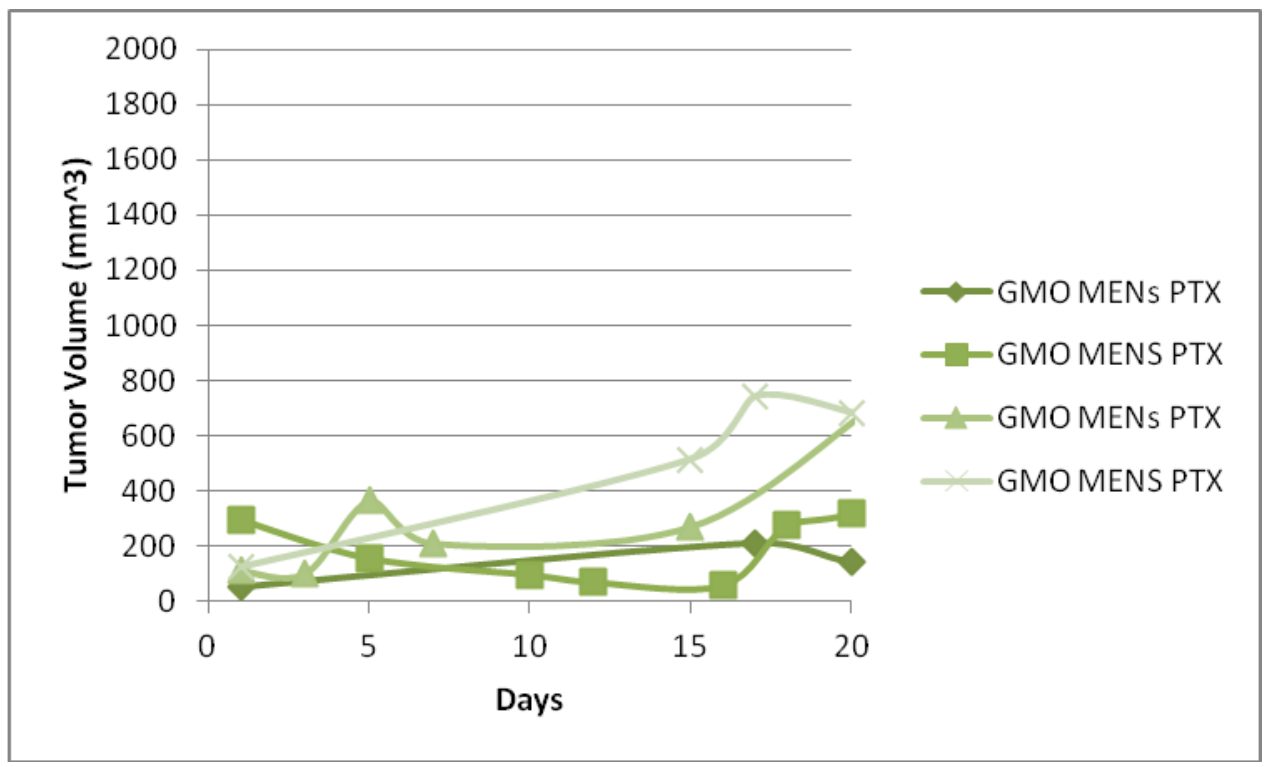

Figure 27. Early MENs-PTX treatment trials.

The first 20 days of the MENs-PTX nanoformulation treatment are shown with identical scaling to establish a direct comparison to positive control mice.

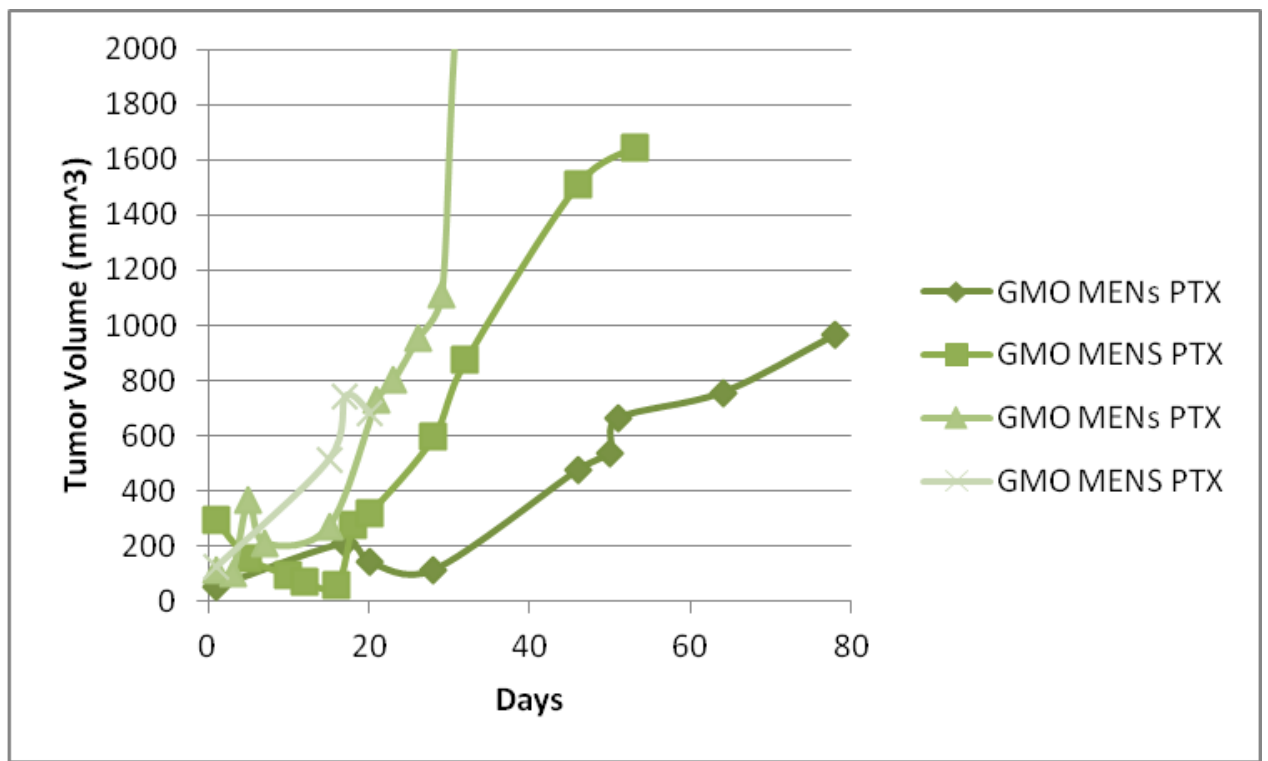

Figure 28. Full MENs-PTX treatment trials.

The full duration of the MENs-PTX nanoformulation trials are shown here. Mice on this treatment were able to maintain good health long after control mice had reached an endpoint. 


\subsubsection{Comparisons of Nanoparticle Delivery Systems}

This set of trials aimed to compare the efficacy of MENs versus conventional MNs and organic PLGA particles, as well as to determine to what extent each of these platforms benefited from active targeting molecules. These experiments utilized the IV injection delivery routes for the nanoformulations. While both PLGA nanoparticles and MNs showed a reduction in tumor development speed, MENs treated subjects showed the best outcomes, including a full eradication of the cancer.

Figure 29 plots the progress of the control groups (a), the MENs experimental treatment groups (b), and the PLGA references groups (c), respectively, all with identical scaling for a direct comparison. Figure 29a shows the most relevant controls to the MENs treatment platform in the form of the positive controls for this trials, blank MN carriers (no PTX), and PTX-loaded MNs (two mice in each group). As expected, the untreated mice had the worst outcomes, followed closely by MNs lacking the active drug in the treatment platform. The two subjects on the MN-PTX platform demonstrated an improved outcome. It should be noted, however, that these subjects were started on their treatment at tumor sizes slightly less than the intended $200 \mathrm{~mm}^{3}$ due to health concerns. Due to the earlier introduction of the treatment, the favorable outcomes are likely to be slightly overestimated for this group. Figure 29b shows the MENs-PTX formulation, where the whole group had performed the best as compared to the other nanoformulations groups. All the mice treated with the MEN-PTX formulation never exceeded tumors volumes of more than twice the starting size (i.e. $400 \mathrm{~mm}^{3}$ ). Interestingly, the addition of targeting molecules did not show any notable improvement of treatment, as the HER-2 antibody, the SPC, and the no-targeting-molecule 
formulations all performed extremely well. In fact, the mouse treated with the non-active targeted MENs-PTX formulation had actually performed the best out of this set; this specimen was fully cured of its cancer using this treatment. The efficacy of the MENs drug delivery platform is then compared to the already well-established PLGA platform (Figure 29c). As expected, PLGA was also a successful cancer treatment platform; however, the PLGA nanocarrier did seem to strongly rely on active targeting molecules to function at the highest efficacy. Both the HER-2 antibody and the SPC ligand showed improved outcomes compared to non-active-targeted PLGA particles, with the HER-2 PLGA-PTX formulation being the only other treatment type in the trial (other than the MENs-PTX formulations) to reduce the tumor size as opposed to simply slowing down growth.

a)

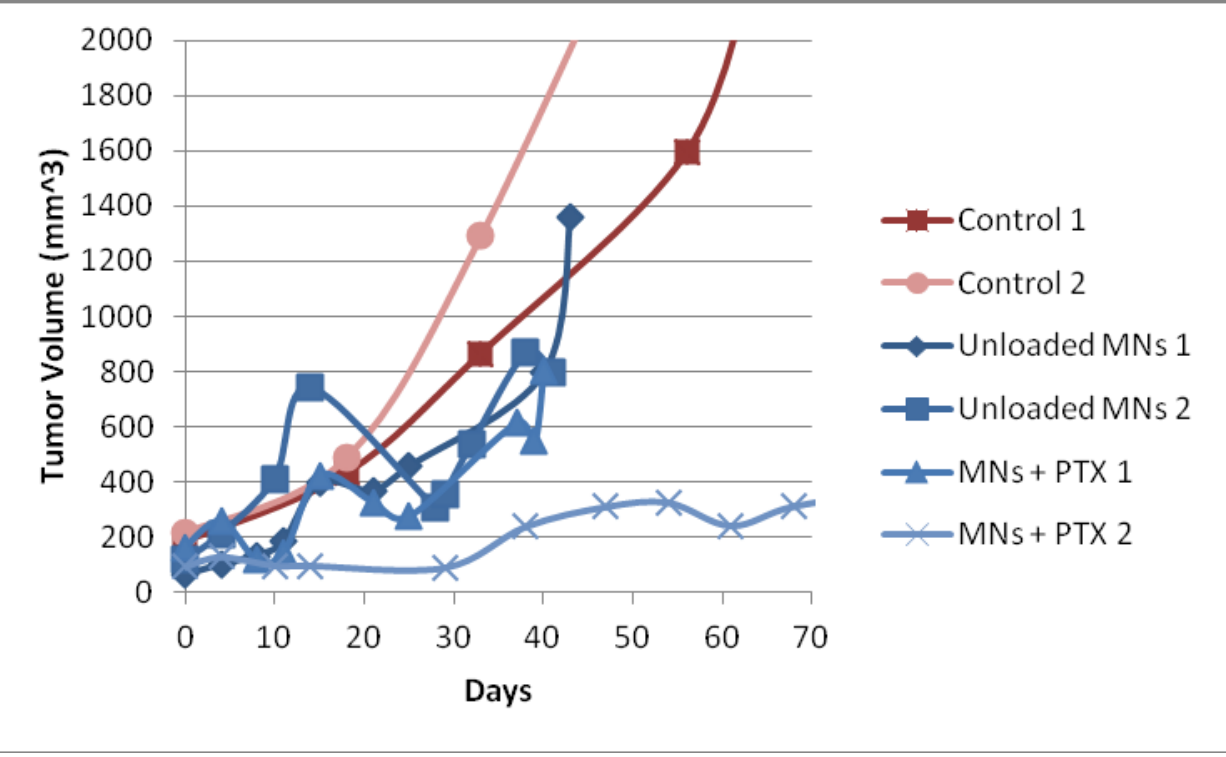


b)

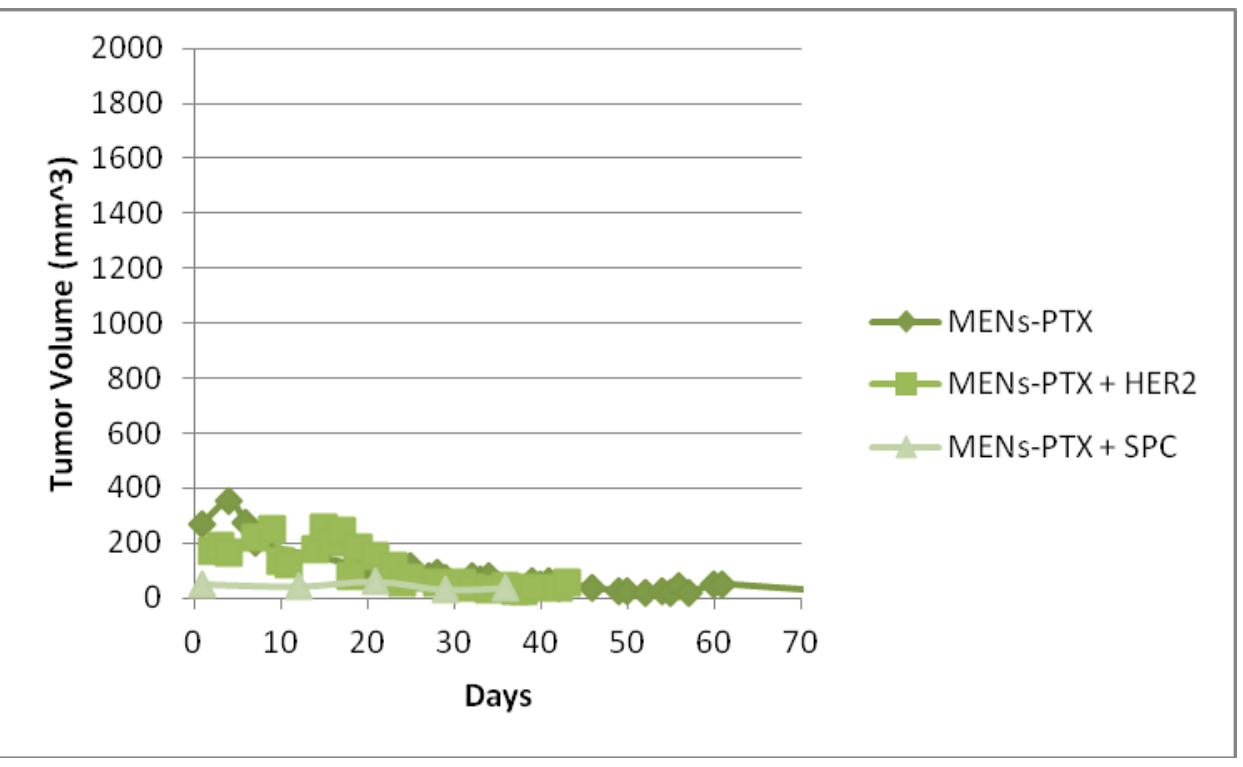

c)

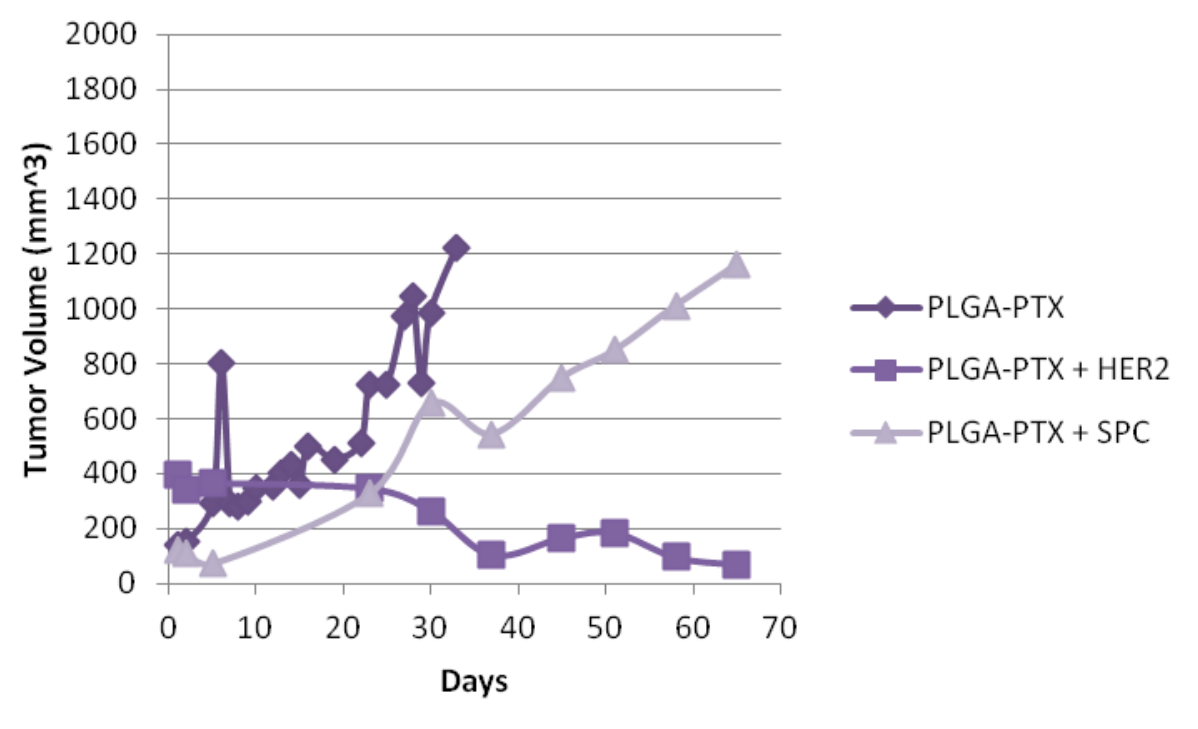

Figure 29. Efficacy of different nanocarrier platforms delivered via IV on tumor volume. a) Untreated tumor growth, blank MN carrier, and PTX loaded MN carrier. b) The MENs-PTX cancer treatment platform, as well as further active targeting with HER-2 and SPC. c) The PLGA-PTX cancer treatment platform, as well as further active targeting with HER-2 and SPC.

\subsubsection{Size and A.C. Field Optimizations of the MENs Treatment}

After establishing that the MENs-PTX platform can effectively treat the cancer, we wanted to investigate if minor changes to the platform offer improved outcomes. 
Specifically, the original trials relied on stimulating the magneto-electric coupling of the MENs with soley a d.c. magnetic field (in the form of the neodymium magntic disk) in order to maintain the simplest form of the treatment for a direct comparison with the currently utlized PLGA-nanoformulation-based treatments, which offer no reliable externally triggered release mechanisms. Furthermore, we were later able to develop a range of sizes for MENs, so we also had the opportunity to investigate if a difference in particle size offered any notable advantages to cancer treatment.

The orgininal MENs were developed to be approximately $30 \mathrm{~nm}$ in size. Therefore, we tested MENs that were approximately three times smaller $(\sim 10 \mathrm{~nm})$ and three times larger $(\sim 100 \mathrm{~nm})$ than the orignial particles. Interstingly, the $10 \mathrm{~nm}$ MENs were too small to establish as strong of a magneto-electic effect as the larger particle sizes, likely due to the amount of surface area versus volume required to do so. For this reason, the $10 \mathrm{~nm}$ MENs exhibited properties somewhere inbetween MNs and MENs, making them an excellent control for particles of identical chemical composition and synthesis technique to MENs, but that display the physical magnetic properties more closely to those of MNs. When injected via IV and activated with the externally affixed magnet, both the $10 \mathrm{~nm}$ and $100 \mathrm{~nm}$ MENs performed acceptably as cancer treatment platforms, as shown in Figure 30; however, the previous trials using $30 \mathrm{~nm}$ MENs were still the most successful. 


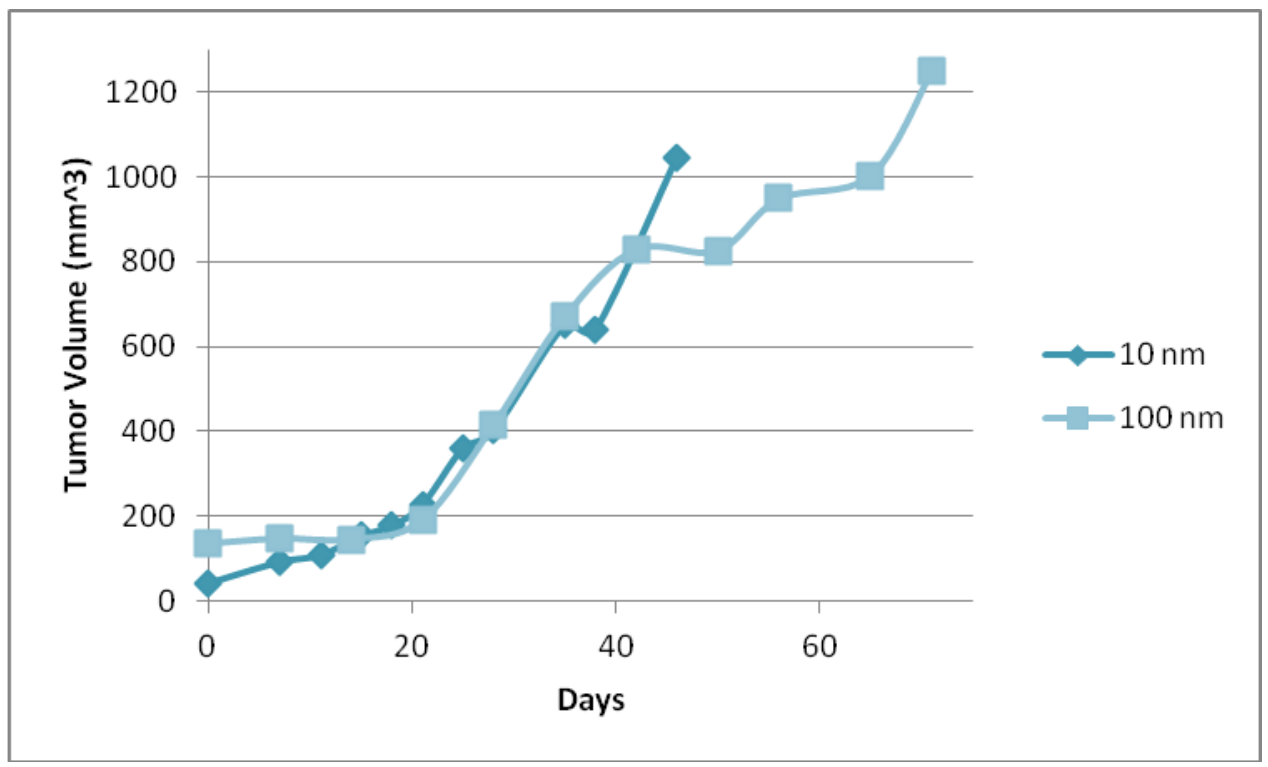

Figure 30. MENs size variants.

Compared to the $30 \mathrm{~nm}$ MENs used in pervious trials, a $10 \mathrm{~nm}$ and $100 \mathrm{~nm}$ variant are tested.

Next, we wanted to incoroporate the manual external release of the payload drug from the MEN carriers as shown in the in-vitro studies (Chapters $3 \& 4$ ) in this in-vivo study. This was accomplished by placing the mouse's cage into a calibrated a.c. electromagnetic field 24-hours after the weekly treatment was administered. The results are shown in Figure 31. The role of the magneto-electric coupling is especially higlighted in these a.c. field trials, where both the $30 \mathrm{~nm}$ and $100 \mathrm{~nm}$ MENs formulations performed extensively better than the $10 \mathrm{~nm}$ MENs. Since the $10 \mathrm{~nm}$ MENs lack a strong magnetoelectric effect, they were not activated any further by the applied a.c. field to release their carried payload, thus an externally triggered payload release was unable to be established and the payload would only be released by the passage of time. By comparison, both the $100 \mathrm{~nm}$ and $30 \mathrm{~nm}$ MENs-PTX treatment groups extensively slowed the growth rate of the tumors. Again, the $30 \mathrm{~nm}$ sized MENs performed the best compared to the rest of the treatment groups. 


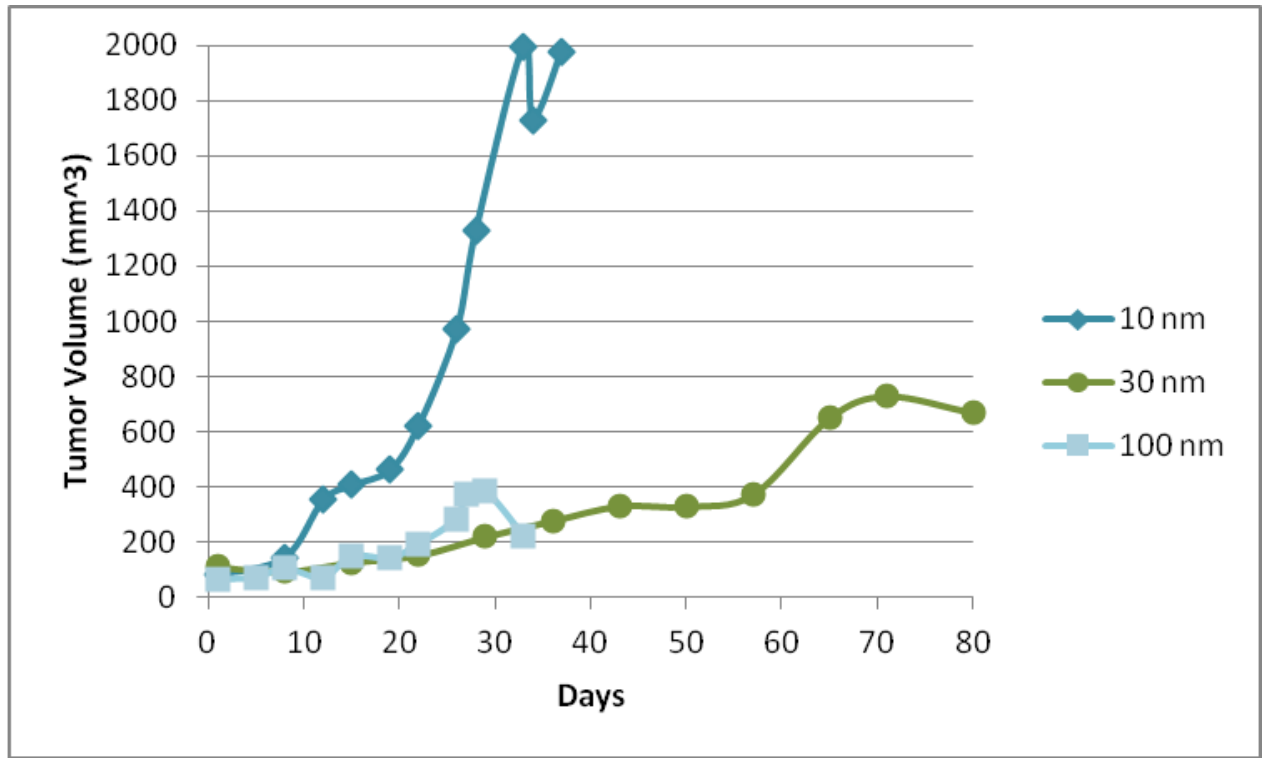

Figure 31. A.C. field application to different sized MENs.

Due to their intrinsic magneto-eclectic coupling, both the $30 \mathrm{~nm}$ and $100 \mathrm{~nm}$ MENs were able to utilize the a.c. field for improved treatment outcomes. $10 \mathrm{~nm}$ MENs were unable to do this as effectively.

\section{$\underline{5.4 \text { Conclusion }}$}

This chapter has shown the first in-vivo demonstration of the efficacy of the MENs platform as a cancer treatment. When compared to conventional magnetic nanoparticles (MNs) and FDA approved organic polymer nanoparticles (PLGA) for reference, the MEN drug delivery platform for PTX was the most effective at treating the model cancer. While both localized and systemic injection delivery of the nanoformulation showed improvement, systemic introduction of the MENs-PTX treatment was able to fully cure all traces of the cancer in the animal model. This was expected, as peritumoral injections could not reach every cell in the tumor mass nor benefit from the EPR effect. 
The most successful mouse trial saw the mouse cured by the $88^{\text {th }}$ day into the weekly treatment (Figure 32), where no tumor could be seen, palpated, or located internally with fluorescent imaging. A live-animal safe florescent tag for the HER-2 receptor was selected to identify the presence of SKOV-3 cells, which greatly overexpress the HER-2 receptor, underneath the outer tissues (skin and fat) that may not be able to be seen or palpated; this was done to confirm a full eradication of the xenografted tumor (Figure 33). The same tag is then used in postmortem tissues examination, along with H\&E staining, to locate any metastasized cancer (Figures 34 and 35). While metastasized SKOV-3 cells were confirmed in the organs of untreated positive control mice, no metastasized tissues we located in MENs-treated mice. This was consistent between H\&E investigated tissues and HER2Sense investigated tissues. Good health was maintained by the MENs-treated mice throughout the treatment, as indicated by weight, physical observation, and active behavior. The cured specimen was observed for three months after the tumor was fully eradicated; the mouse had gained about $20 \%$ of its body weight (a favorable outcome) and demonstrated perfect physical health, which was supported by behavioral tendencies to be active, curious, and nesting. 

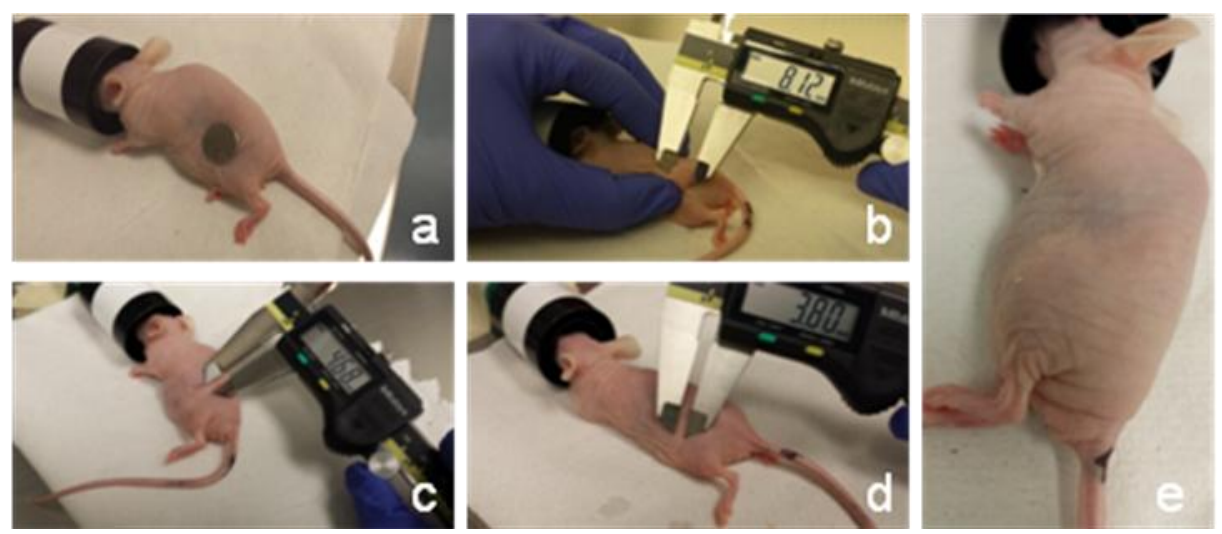

Figure 32. A fully cured mouse's progress.

a) The mouse is shown wearing the d.c. magnetic field generating magnet. b) The volume of the tumor at the start of treatment was $286 \mathrm{~mm}^{3}$. c) The tumor volume was $51 \mathrm{~mm}^{3} 35$ days into the treatment. d) The tumor volume was at $20 \mathrm{~mm}^{3} 57$ days into the treatment. e) There was no tumor remaining 88 days after the treatment had begun.

a)

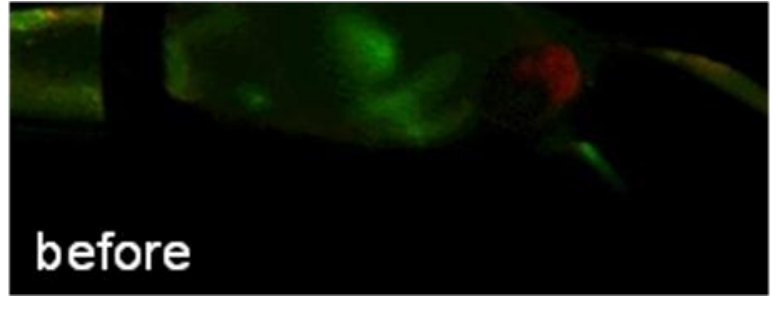

b)

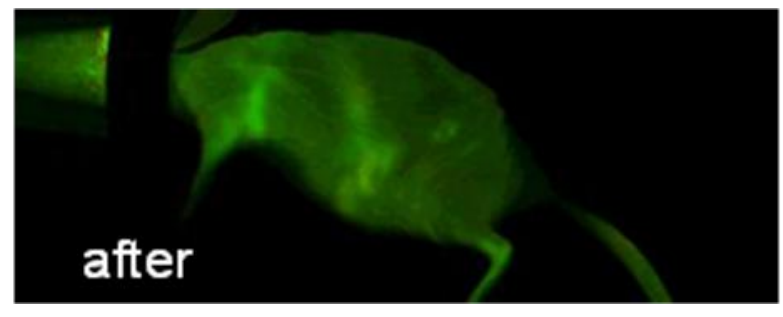

Figure 33. HER2Sense monitoring of live animals.

a) Tissues from an untreated mouse are shown to demonstrate that metastasis to the kidneys had occurred. b) Tissues from treated mice are shown to demonstrate that none of the investigate organs showed signs of metastasized cancer nor any changes from healthy morphology. 


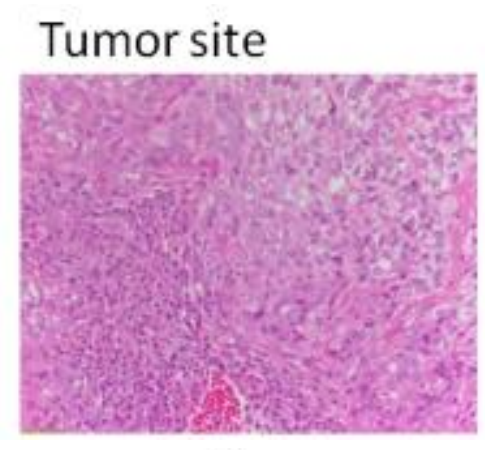

a)

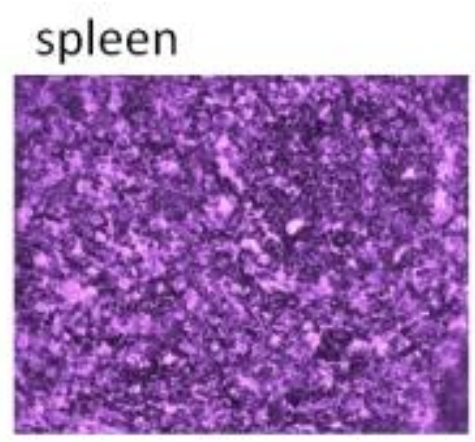

\section{lung}

b)

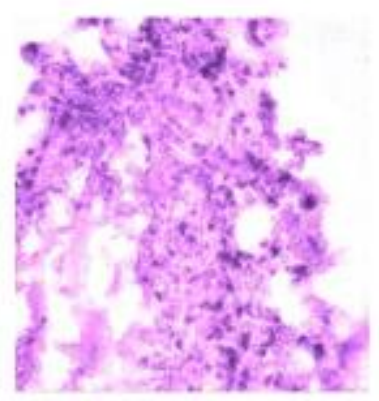

\section{kidney}

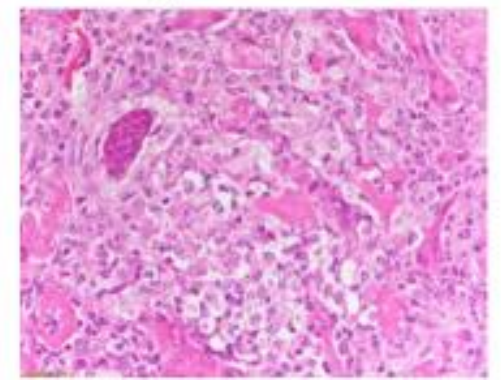

\section{kidney}

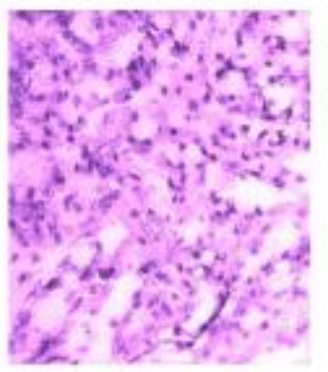

\section{liver}

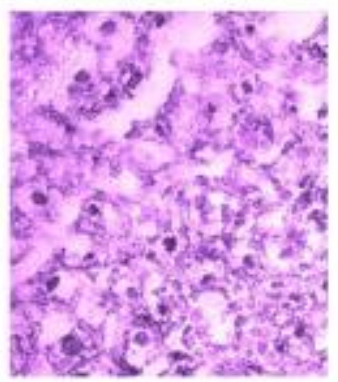

Figure 34. H\&E stained organs.

a) Tissues from an untreated mouse are shown to demonstrate that metastasis to the kidneys had occurred. b) Tissues from treated mice are shown to demonstrate that none of the investigate organs showed signs of metastasized cancer nor any changes from healthy morphology. 

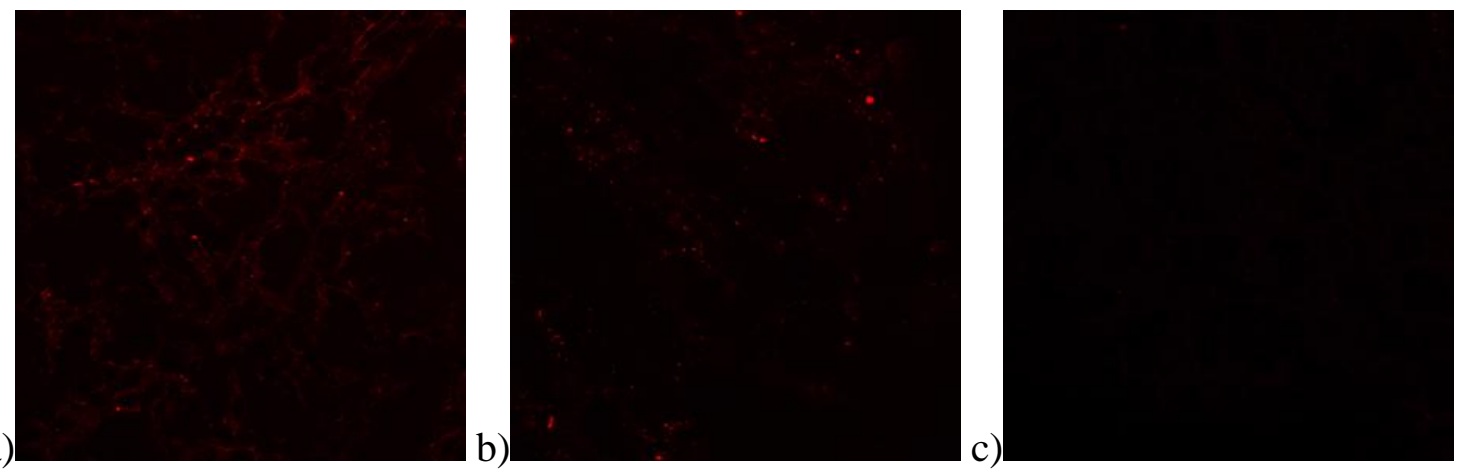

Figure 35. HER2Sense stained organs.

a) Tumor tissue of an untreated specimen. b) Kidney tissue of an untreated specimen. c) Kidney tissue of a treated specimen, showing no presence of HER-2 and therefore no metastasis

Only the MENs-PTX formulations were able to reduce the tumor size enough to ultimately cure the cancer, although the HER-2 antibody active targeted PLGA-PTX particles also reduced tumor volume. The PLGA platform's reliance on active targeting molecules makes it more sophisticated to formulate and less generalizable to different cancers. PLGA not tagged with further targeting molecules rely mostly on the passive EPR effect, which is why notable improvement was observed upon the introduction of a surface targeting molecule that enabled the platform to utilize active targeting. What may be occurring is that without the active targeting to speed up the tumoral localization, the PLGA particles had broken down and released their carried payload before reaching the tumor site, thereby not delivering some of the treatment to the cancer. By comparison, the MENs did not rely on active targeting, although they likely also benefited from it at least somewhat. Presumably, the forces of attraction to the electrical aspects of the cancer cell membrane are comparably strong to the forces of attraction of the antibody and ligand to their respective surface proteins. 
The electric properties unique to MENs also enable the application of a small external a.c. magnetic field to be used to control the timing of the payload release from the MENs. While time will also eventually release a drug from a nanocarrier, the ability to be able to do so as soon as needed is highly beneficial for customizing individual treatments. Mice under a.c. magnetic field treatment following the regular treatment regime (nanoformulation injection + d.c. magnetic field) responded positively to the treatment. Both the d.c. field and a.c. fields used in this mouse study can easily be scaled up for human use with strategic use of solid magnets or magnetic field generator setups.

This study was the very first of its kind, and because of this the number of animals used was deliberately kept minimal. Furthermore, to meet or exceed animal husbandry standards, each animal was treated as indicated by its health and well-being above as indicated by the experiment. For this reason, some of the treatments started earlier than the initially defined starting point of $200 \mathrm{~mm}^{3}$. Additionally, animals were euthanized if found to be in distress, forcing some of the trials to stop earlier than desired. While a larger study would undeniably be ideal, this mouse study answered the most pressing question regarding this novel cancer treatment platform, and that was "does the efficacy of the MENs cancer treatment platform translate from in-vitro to in-vivo?" with a definitive "yes." 


\section{CHAPTER 6: BIODISTRIBUTON AND CLEARANCE OF MENS}

\section{$\underline{6.1 \text { Introduction }}$}

In this chapter we investigated the biodistribution and clearance of our novel nanoformulation, MENs, for the very first time in an animal model. We employed a new SEM/EDS based technique to systematically map out nanoparticles in tissues. In this study, we specifically compared the biodistribution of mice being treated for cancer to the biodistribution of mice that do not have cancer. We have found that the nanoformulation accumulates in the tumor preferentially, however it is also important to document where the MENs would travel if there are no cancer cells to selectively uptake it. This is especially relevant for post-treatment clearance after the course of treatment is completed. Also, since MENs can be used as a drug delivery platform for treatments other than cancer, this information is pertinent to other potential nanoformulations with MENs for other medical uses. To accomplish this, we compare the organs of the cancertreated mice from Chapter 5 to a cancer-free cohort. In order to investigate the long term trajectory of the MENs, the cancer-free group was further divided into three time periods: 1 week, 1 month, and 2 months. Four different nanoparticle sizes were investigated for size related differences, including MENs sized at $10 \mathrm{~nm}, 30 \mathrm{~nm}, 100 \mathrm{~nm}$, and also new, larger MENs sized at $\sim 500 \mathrm{~nm}$. This was done to establish a sort of stop-motion based time line showing where the particles go soon after administration, where they remain, and where they go en route to being cleared, as affected by the particle size. 


\section{$\underline{6.2 \text { Experimental Procedures }}$}

All the protocols remain unchanged from the experimental procedures described in Chapters 4 and 5 to maintain the highest level of consistency.

\subsubsection{MENs Preparation \& Functionalization}

$\mathrm{CoFe}_{2} \mathrm{O}_{4} \mathrm{MENs}$ were synthesized via the hydrothermal method as described in Chapter 4.2.1 and functionalized with a $2 \mathrm{~nm}$ thick layer of GMO as described in Chapter 4.2.2. This was done identically for the $10 \mathrm{~nm}, 30 \mathrm{~nm}, 100 \mathrm{~nm}$, and $500 \mathrm{~nm}$ MENs used.

\subsubsection{General Animal Care}

Mice were cared for as described in Chapter 5.2.4.

\subsubsection{Mouse Treatment}

Two groups of mice were used for this experiment:

1) The mice investigated for cancer treatment in Chapter 5 were used for the cancer group in this study to minimize redundant animal use. Specifically, the application of magnetic fields was investigated as related to the trajectory of the MENs throughout the physiology.

2) A group of identical mice from the same immunocompromised strain was used for the general biodistribution and clearance group. These mice were randomly assigned a nanoparticle size and clearance duration. The mice were given a single bolus lateral tail vein IV injection of $5 \mathrm{mg}$ GMO-MENs (there was no drug conjugated to the nanoparticles) suspended in $200 \mathrm{ul}$ sterile PBS. A high concentration was deliberately selected to best demonstrate the effects of the nanoparticles after a single treatment and to 
make the data more comparable to the cancer group, which had received continuous weekly treatments.

\subsubsection{Tissue Collection \& Preparation}

The organs excised for biodistribution and clearance studies were the liver, lungs, kidneys, and spleen, as these are the organs most commonly associated with inorganic nanoparticle accumulation [74]. The brain was also collected for examination.

Animals were euthanized and the tissues were processed as described in Chapter 5.2.8, minus the addition of coverslips and the histology staining protocol. Immediately prior to SEM imaging, the slides were gently rinsed with PBS to remove any excess paraffin from the tissues and allowed to dry for improved definition.

\subsubsection{SEM Imaging with EDS Analysis}

For SEM imaging, tissue slides were gold-sputtered for 75 seconds, grounded with copper tape for proper conductivity, and imaged using a JEOL- JIB 4500 Multibeam system (FIB/SEM) with a Thermo-Scientific Noran System 7. Back-scattered electron detection mode was used to locate areas of high densities of heavy metals under which MEN clusters would fall. Then, each nanoparticle cluster candidate was investigated using the "point-and-shoot" mode of the energy dispersive spectroscopy (EDS) machinery, specifically for the elements barium (Ba) and titanium (Ti). While many heavy metals are naturally occurring in physiology, such as iron [75] and calcium [76], the MEN perovskite shell composing elements of $\mathrm{Ba}$ and $\mathrm{Ti}$ are relatively rare. Thus, EDS-identified $\mathrm{Ba}$ and Ti confirms a MEN deposition point. 
SEM/EDS image quantification was performed using NIH developed ImageJ software [77]. This software calculates the percentage of the tissue area that contains MENs. Multiple images that are the best representatives of the biodistribution in the organ were selected for quantification. The images were scaled to match and the software's identification threshold was manually adjusted to correctly identify the MEN clusters specifically in the areas of the tissues (i.e. discriminate between tissues on the slide versus the glass slide itself). The quantifications were then averaged between all analyzed images and plotted.

\section{$\underline{6.3 \text { Results }}$}

\subsubsection{MEN Biodistribution and Clearance in Mice Treated for Cancer}

Using SEM imaging with EDS analysis, we are able to detect MENs inside the tumor tissue of MEN nanoformulation treated mice. This confirms that the MENs actually enter the tumor mass, past the outer tumor capsule, to deliver the treatment internally (rather than just washing the tumor with the treatment). Furthermore, an applied magnetic field amplifies this effect.

Figure 36 shows an example of the EDS analysis on a cluster of MENs in tumor tissue. As seen in Figure 36b, the elements with consistent signal distribution throughout the whole surface were not concentrated at any one point. Silicon ( $\mathrm{Si}$ ) was overrepresented on areas of the slide where tissue was absent as the signal was coming from the glass slide itself, while Ba and Ti show MENs localized on the tissue portions (not on the Si heavy glass portions) of the slide. Figure 36c gives a quantitative comparison of all the elements present in the EDS analyzed image from Figure 36a. 

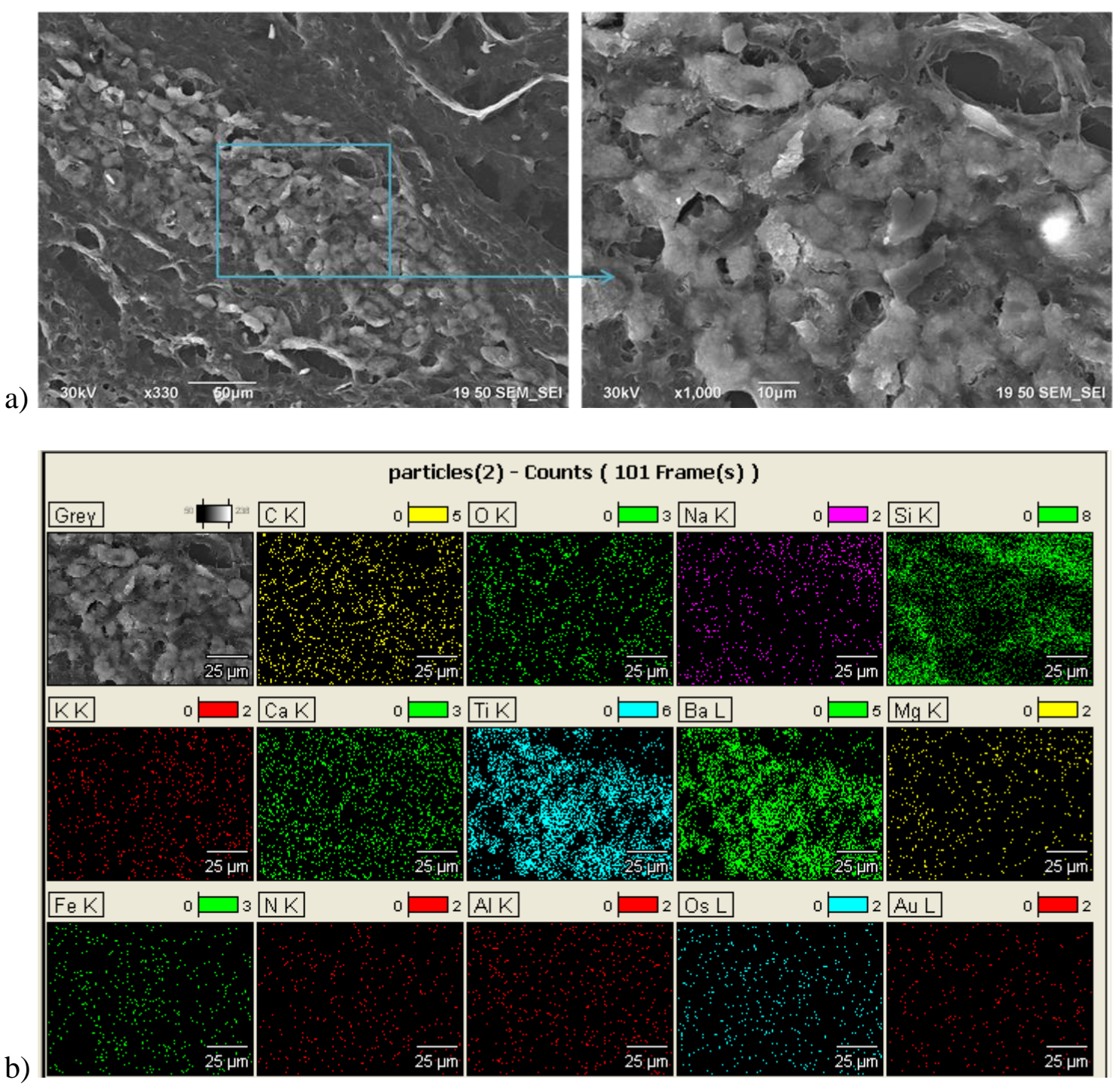


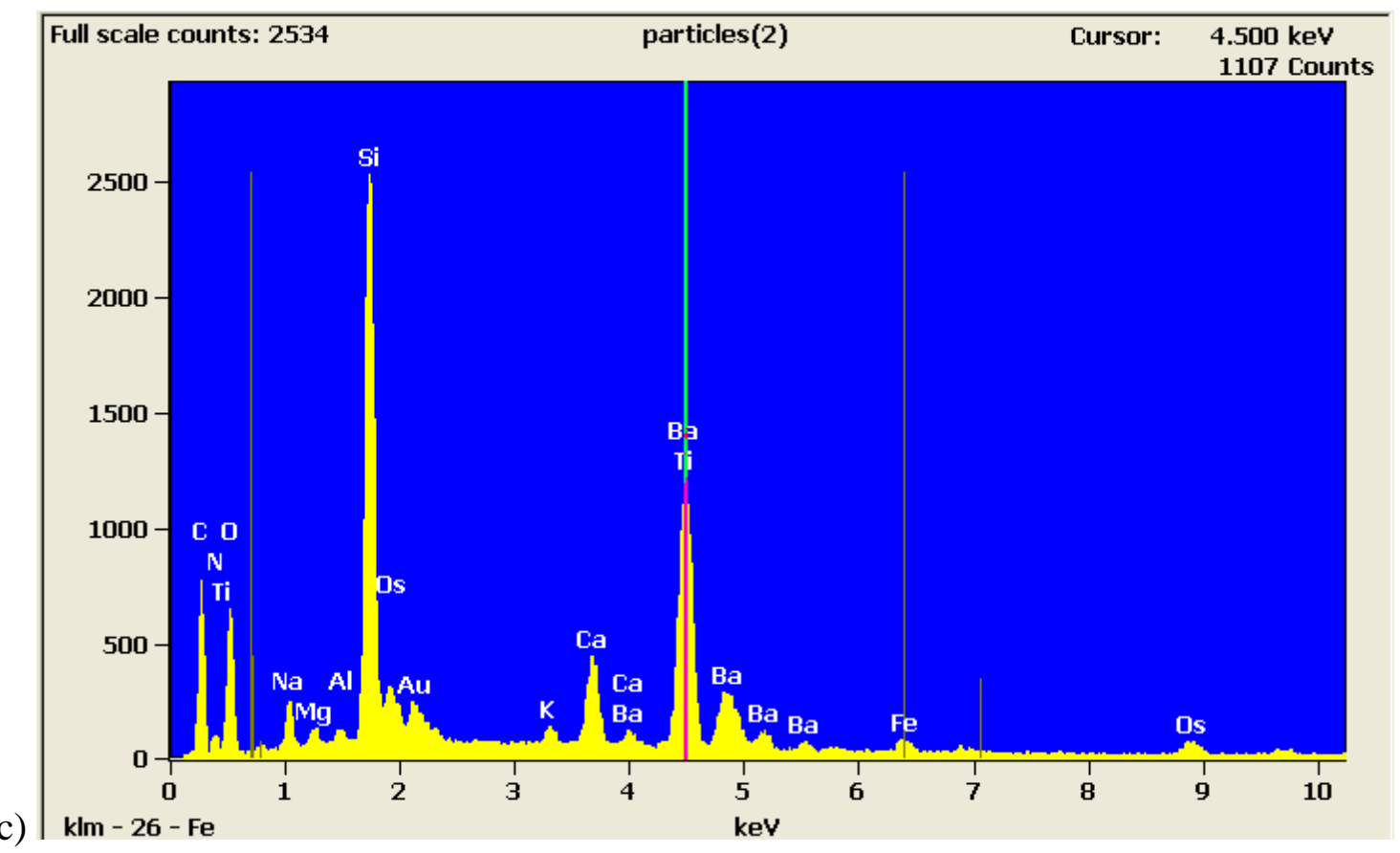

Figure 36. EDS analysis for MENs presence in tumor tissue.

a) The backscattered view of the tumor tissue is shown on the left. On the right is the magnification used for EDS analysis. b) The elements EDS was used for are shown here. c) The elements EDS detects are quantified here. The green vertical line highlights the peak that represents $\mathrm{Ba}$ and $\mathrm{Ti}$, as indicative of MEN presence.

An interesting observation was that the clusters of MENs in the tumor tissue were not haphazard in distribution, and instead followed the contours of the tumor's tissue morphology, as seen in Figure 37. For mice treated with a subcutaneous injection of the MENs, most of the clustering occurred towards the periphery of the tumor, as expected. By contrast, IV treated mice had MEN clustering throughout the entire tumor, which likely explains the improved efficacy of IV versus subcutaneous injection described in Chapters 5.3 and 5.4. This helps to demonstrate that the entry point of the MENs into the tumor, with the IV entry points being essentially limitless throughout the tumor while the subcutaneous entry points are limited to the periphery and are therefore much slower to reach the core of the tumor, ultimately helps determine the efficiency of tumor reduction. 
This was consistent with the subcutaneous nanoformulation treated mice showing a reduction of tumor growth speed (as the treatment whittles away at the outside of the tumor mass) while the IV treatment works to target the tumor cells inside-out for a notable deterioration of the tumor mass. This concept is illustrated in Figure 38. The subcutaneous injection, while demonstrating a positive effect, was not able to notably shrink tumors because the tumor tissue furthest away from the periphery is able to continue growing even while the periphery is being affected by treatment. On the other hand, systemically administered treatment treats the whole tumor, thus mostly affecting the areas that are best perused and that are, therefore, the most likely to have grown the most robustly in the absence of the treatment. 
a)
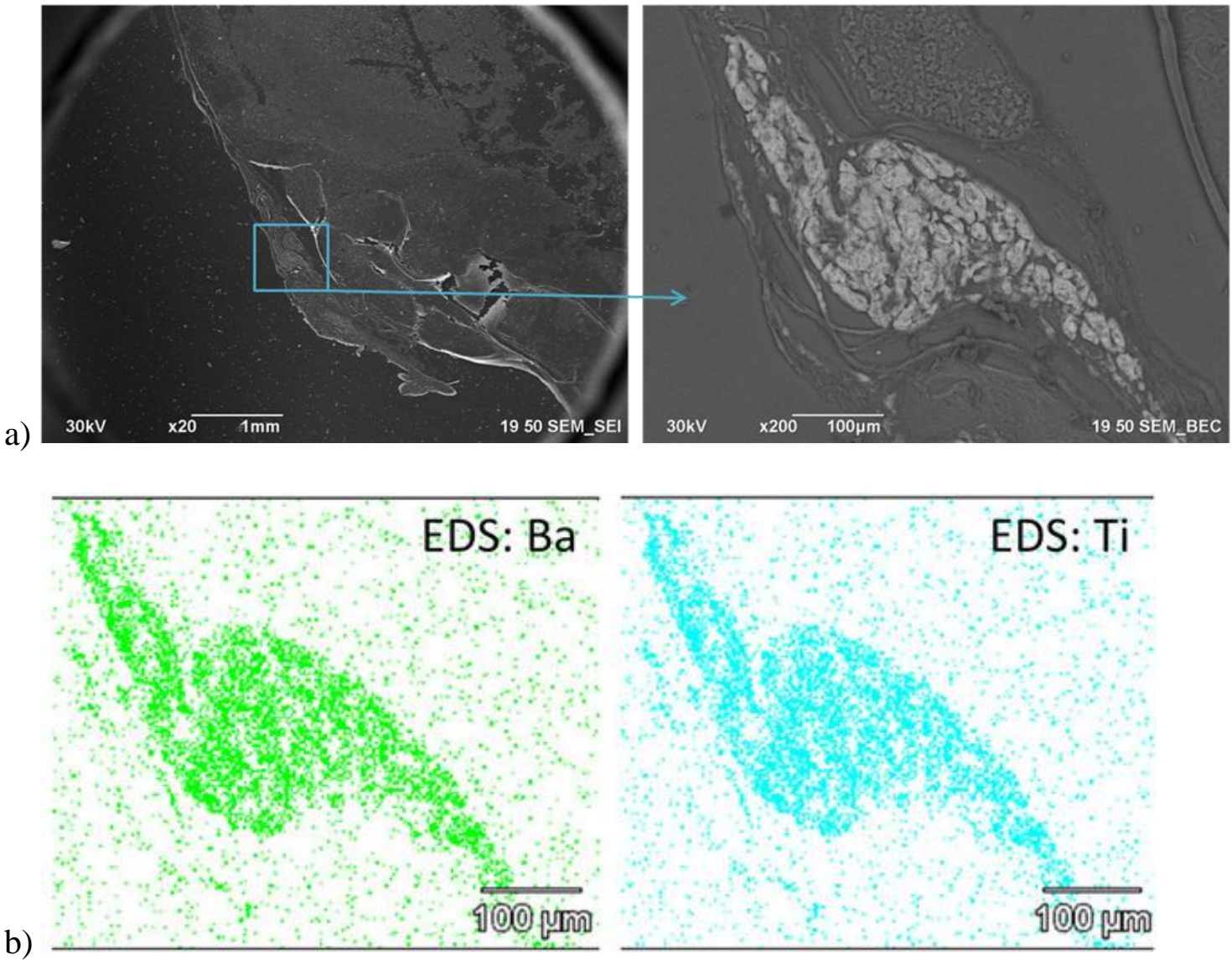

Figure 37. EDS located MENs follow tumor morphology.

a) Back-scattering mode SEM images show the tumor morphology. b) Ba and Ti confirm the identity of MENs in the tissue as they fit perfectly in the contours of the tumor tissue. 


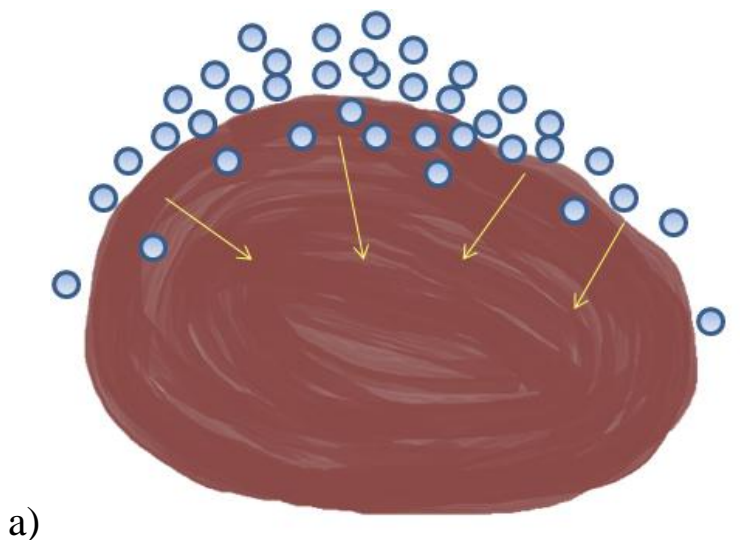

b)

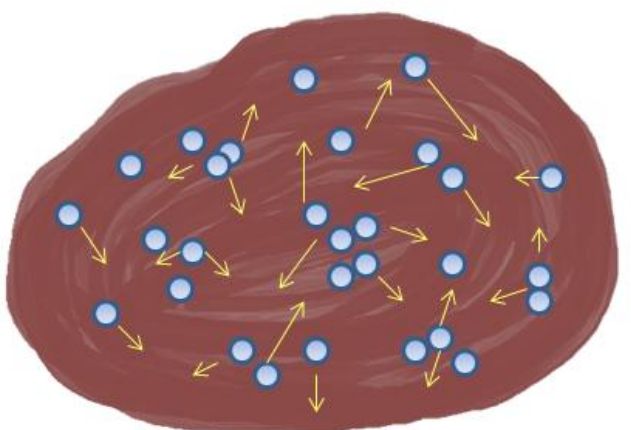

Figure 38. Subcutaneous vs IV localization of MENs.

The red mass demonstrates an encapsulated tumor. The blue spheres represent the MEN treatment, with the yellow arrows projecting the trajectory of the treatment throughout the tumor mass. (a) While the entirety of the treatment is available in the immediate vicinity of the tumor, subcutaneously administered treatment suffers from having to internalize past the outer tumor capsule and being delayed from reaching the innermost cells of the tumor. (b) By contrast, IV administered treatment has an available tumor entry point anywhere tumor cells are vascularized. This lets the treatment enter multiple areas of the tumor simultaneously and begin eradicating cells throughout the entire tumor, which accomplishes an overall reduction of tumor mass.

In addition to the nanoformulation administration method having notable biodistribution differences, it was also not surprising to see that the incorporation of an externally applied magnetic field also modified the biodistribution. The application of a d.c. magnetic field on the order of 100 Oe in the form of a magnetic coin affixed to the tumor site enhanced the localization of the MENs into the tumor preferentially over the peripheral organs, as shown in Figures 39 and 40. These charts are drawn from three representative mice under different cancer treatment conditions: (i) a fully cured mouse that has completed its course of MEN-PTX nanoformulation treatment and was observed for three months after the final treatment was administered before being euthanized for biodistribution studies; (ii and iii) two mice in the very early stages of treatment (two weekly administrations into treatment) were used as positive controls demonstrating the 
biodistribution when the tumor was at its largest, peak value. (ii) One mouse had no further tumor targeting, while the other (iii) had a magnetic coin affixed to the external surface of the tumor to establish a magnetic field of approximately 100 Oe directly at the tumor site.

In the magnetic field treated mice, a little under a third of the MENs were localized in the tumor. This contrasts with the non-magnetic field treated mice, which contained approximately a tenth of the total MENs in the tumor mass (as opposed to in the peripheral organs). The field treated mice overall had the majority of the MENs localized in the tumor tissue, while non-field exposed mice saw the majority of the MENs localize in the liver and lungs, followed by the spleen, and only then the tumor. The cured mice had no tumor to house MENs, however, these mice also had a very small fraction of MENs present throughout the periphery as well. This is presumably because the three month period after the cessation of MEN administration had allowed most of the MENs to clear from the mouse unassisted. Other than the strong preference for the tumor, the proportion of distribution throughout the peripheral organs was similar between the field and no-field treatment groups, as would be expected of nanoparticles of identical size and composition. This distribution pattern helps demonstrate the unique role of the magnetic field in this type of cancer treatment. 


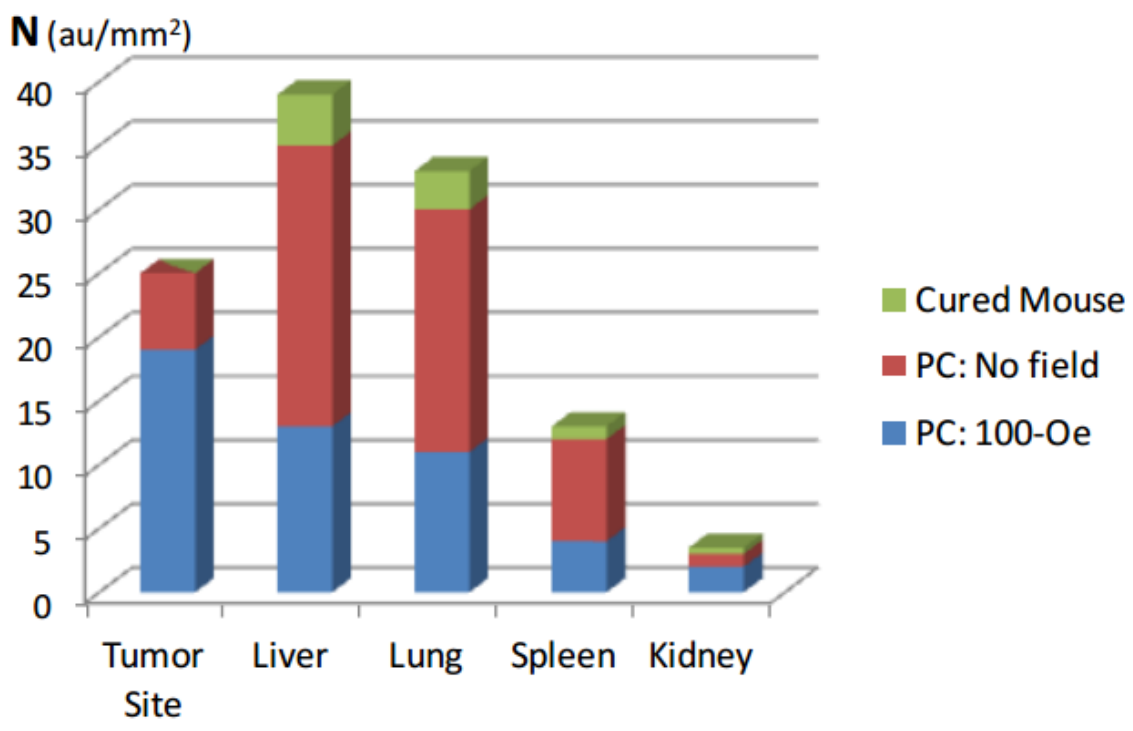

Figure 39. MEN distribution in cancer treated mice

This chart shows the areal concentration of MENs in the tumor site compared to peripheral organs sites (liver, lung, spleen, and kidney) as affected by the MEN treatment conditions: fully cured, under treatment with no applied magnetic field, and under treatment with an applied magnetic field.

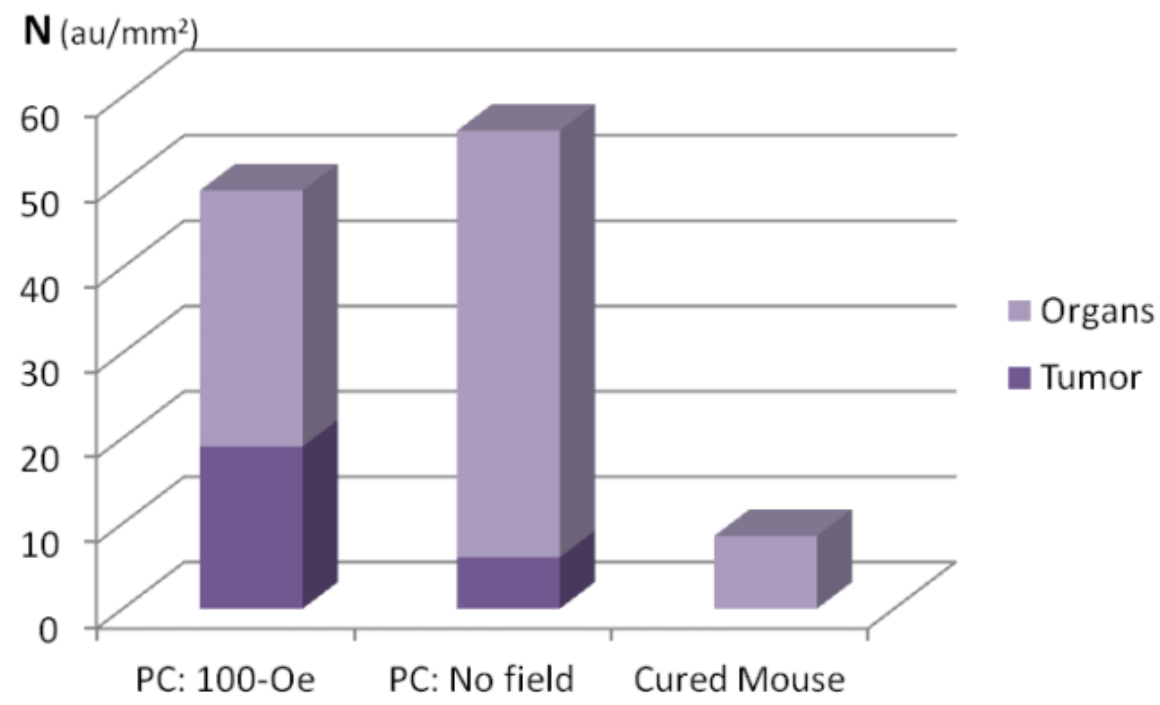

Figure 40. Overall organ vs tumor MEN load in MEN treated mice.

This chart shows a comparison between the areal concentration of MENs in the tumor versus peripheral organs (liver, lungs, kidneys, and spleen combined) of a mouse being treated for cancer with an applied magnetic field, a mouse being treated for cancer without an applied magnetic field, and a mouse that has been successfully treated of its cancer. 


\subsubsection{MEN Biodistribution and Clearance over Time}

\section{Biodistribution \& Clearance through the Liver, Lungs, and Spleen}

The liver, spleen, and lungs had easily locatable MEN clustering throughout all the tested time points. Figure 41 shows the gradual clearance of the MENs from these organs over time by maintaining a constant scale for reference. Figure 42 shows a close up comparison to help visualize the concentration of the different particle sizes in the organs over time.

As seen in Figure 41, after one week following administration, most of the MENs were located in lung tissue, followed by the spleen, with very little found in the liver. This contrasts with the one month post-injection time period, where MEN (sizes $10 \mathrm{~nm}$ through $100 \mathrm{~nm}$ ) presence had actually grown in the liver; this was the only documented instance in the trial where the concentration of MENs in an organ increased over time. What appears to be happening is that the large initial concentration of particles in the lungs travels to the liver by means of hepatic clearance, as indicated by the greatest reduction of particle concentration in the lungs and the greatest increase of particle concentration in the liver. The spleen had a less dramatic reduction in particle concentration from one week to one month post-administration. Then, at two months after the bolus administration, particles at all organ sites had reduced further, indicating a successful gradual clearance process of all the tested sizes. 
a)
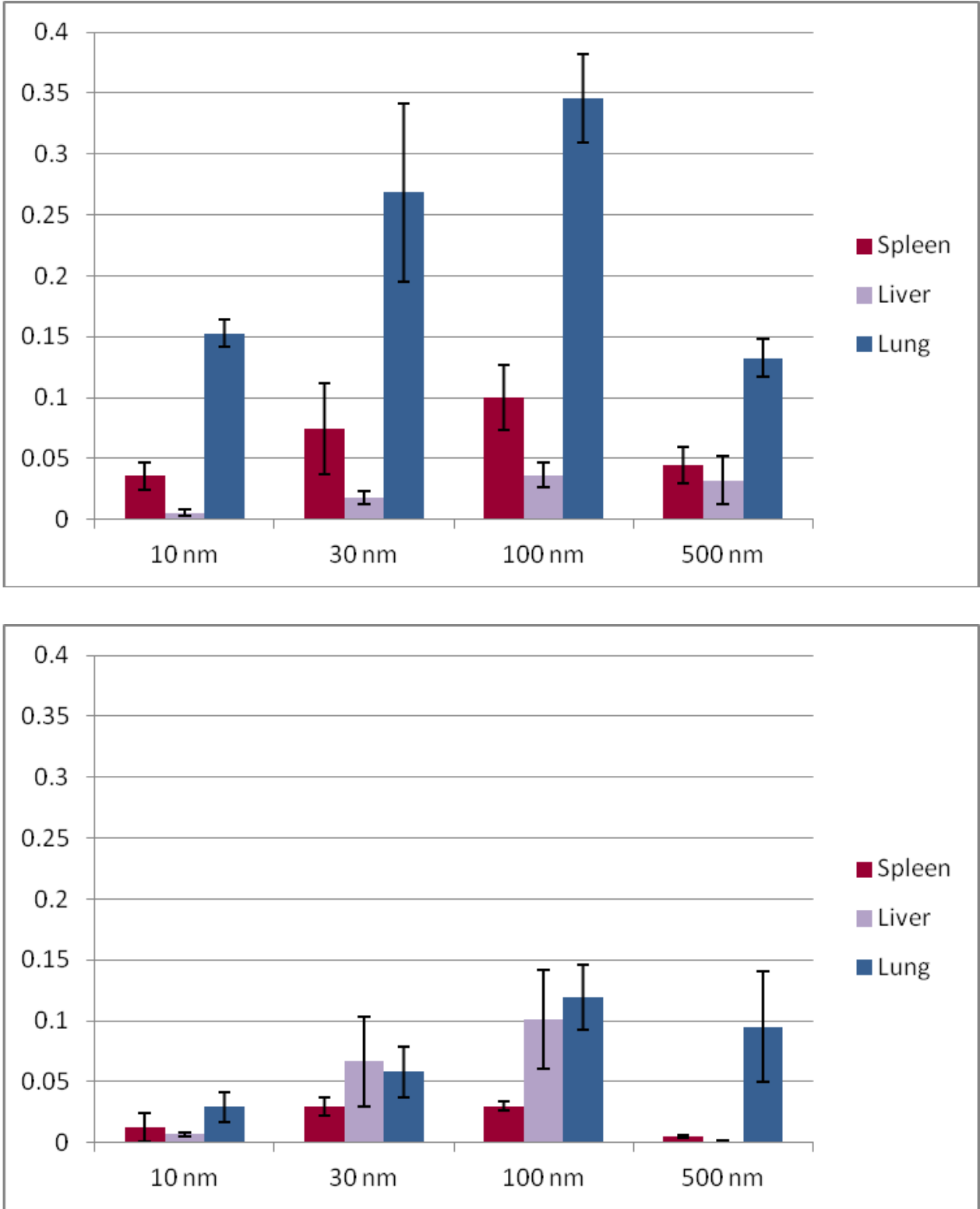

b) 


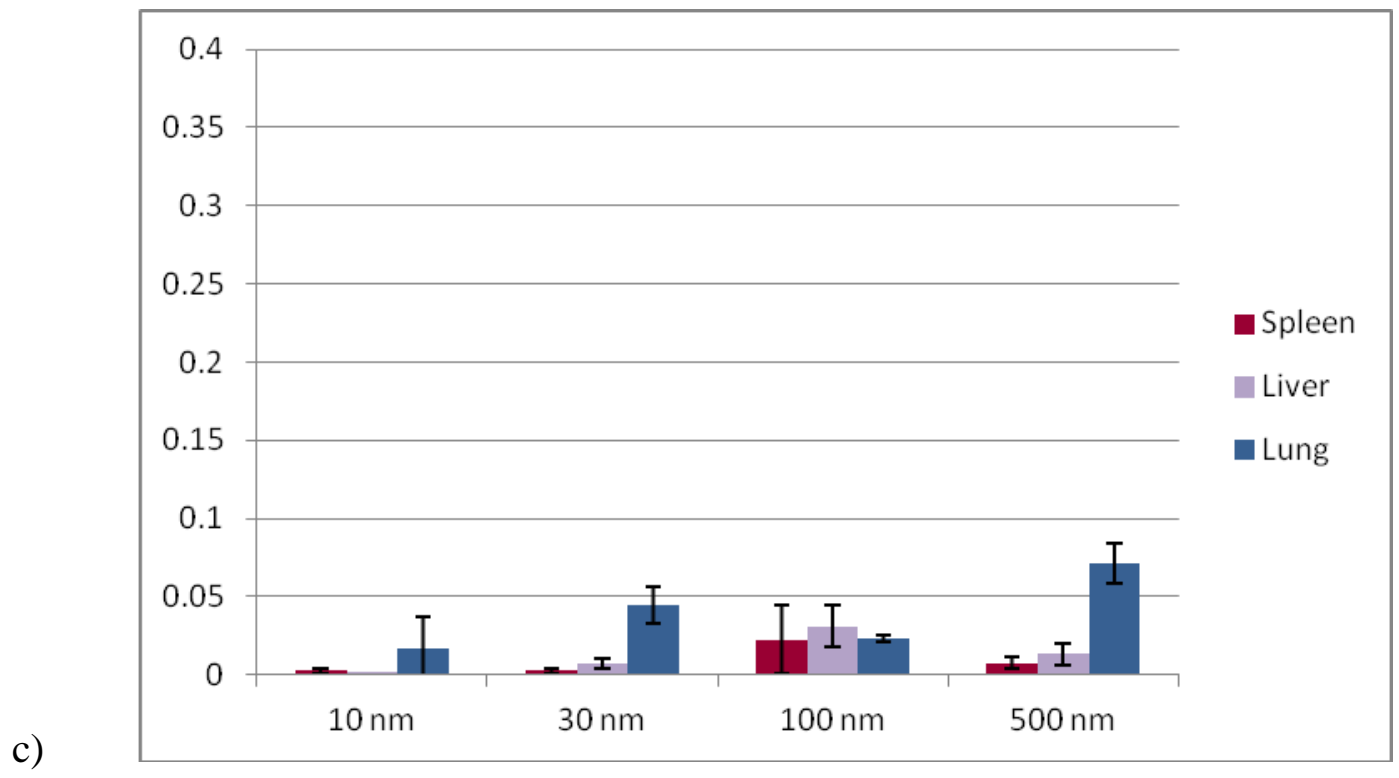

Figure 41. MEN clearance over time.

The vertical axis is MEN cluster absorbance signal per tissue area. a) 1 week post injection b) 1 month post injection c) 2 months post injection.

Figure 42 focuses more on demonstrating the changes in the organ localizations between the different particle sizes, considering that in all instances the overall nanoparticle concentration reduced over time. The growth of MEN presence in the liver for $10 \mathrm{~nm}, 30 \mathrm{~nm}$, and $100 \mathrm{~nm}$ MENs is very clear here between the one week (42a) and one month (42b) post-injection period. Interestingly, $500 \mathrm{~nm}$ MENs were not highly present in the liver or the spleen at one month-post injection, and remained at a high concentration in the lung throughout the course of observation. Overall, the $10 \mathrm{~nm}$ MENs were the least present in the studied organs at all times throughout the study (possibly because they were eligible for renal clearance, as discussed in the next section), and were likely to be the first to completely clear from the body. By contrast, the $500 \mathrm{~nm}$ particles were likely to be the slowest to clear, with the $30 \mathrm{~nm}$ and $100 \mathrm{~nm}$ falling in order to establish the pattern that the smallest and largest particles were fastest and slowest to clear, respectively. What appears to have happened was that the initial deposition of 
MENs occurred mostly in the lungs and in the spleen; then the MENs in the spleen are slowly processed for biological clearance while many of the MENs in the lung transitioned to the liver, as well as the spleen to a lesser extent. This process then continues until the particles are fully cleared out of the biological system, which would presumably take a total of 3-4 months depending on the particle size.

a)

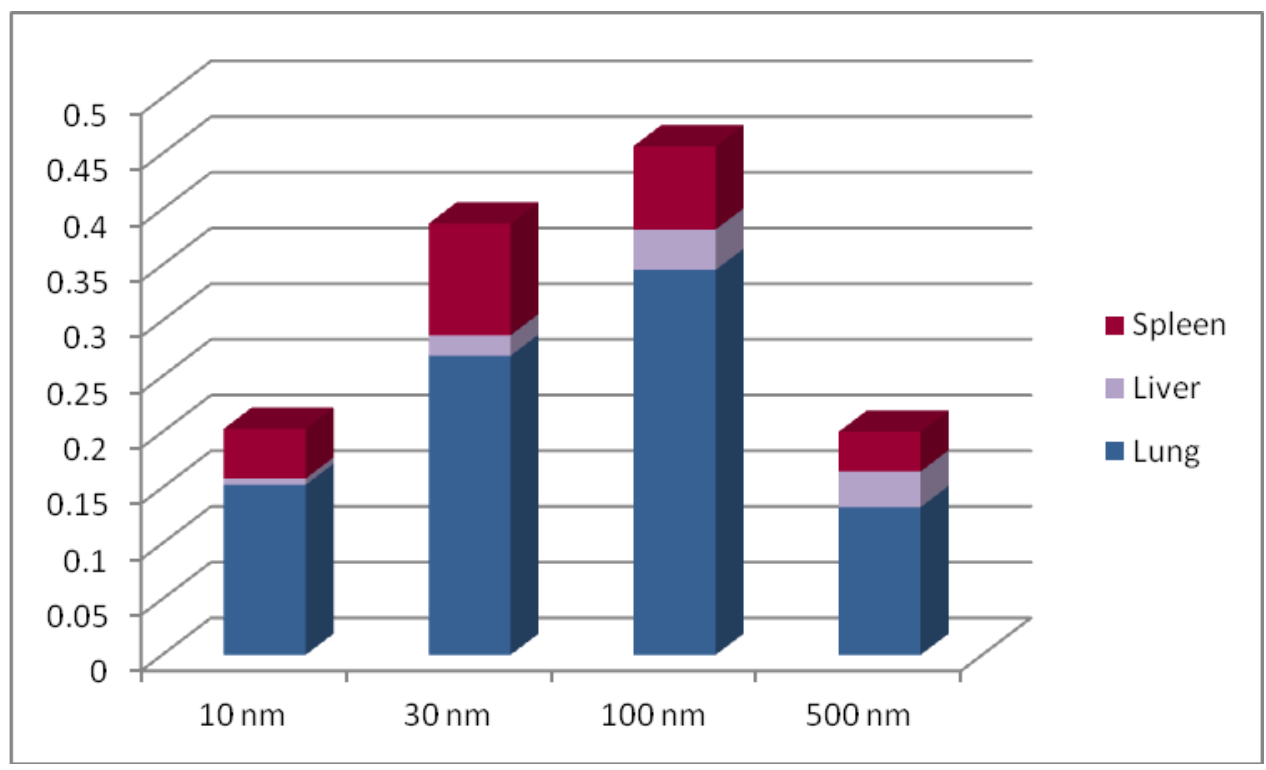

b)

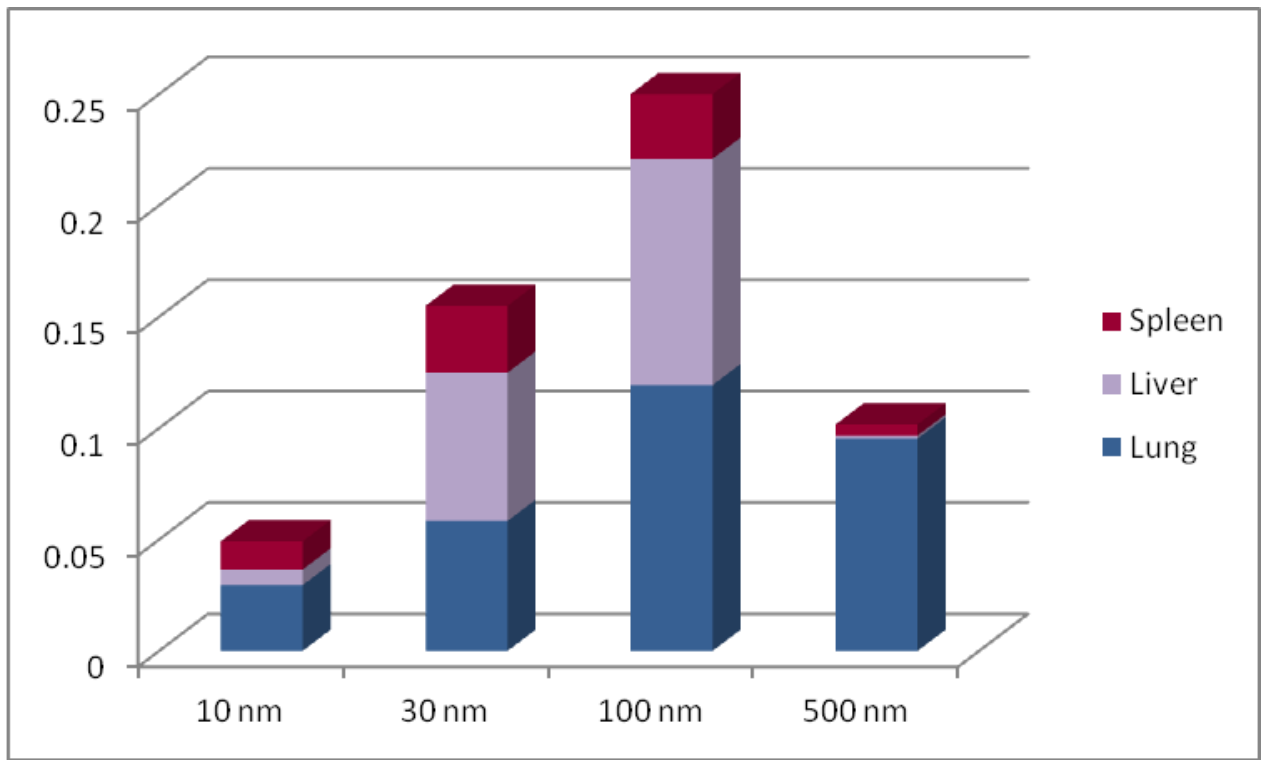


c)

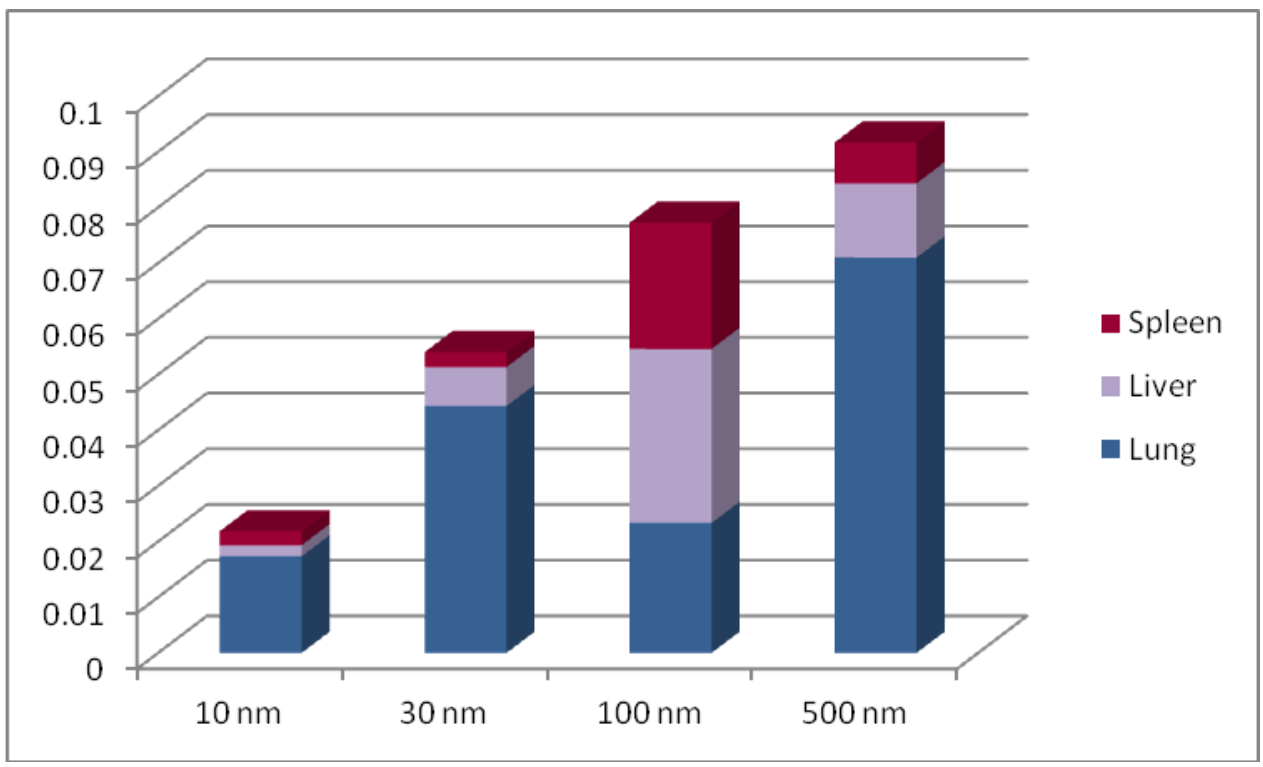

Figure 42. MEN organ distribution over time.

The vertical axis is MEN cluster absorbance signal per tissue area. a) one week post injection b) one month post injection c) two months post injection.

\section{Biodistribution \& Clearance Considerations of the Kidneys}

The presence of MENs in kidney tissue was so minimal that it was difficult to quantify. Very few MEN clusters could be identified, but a region positive for MEN clusters is shown in Figure 43.
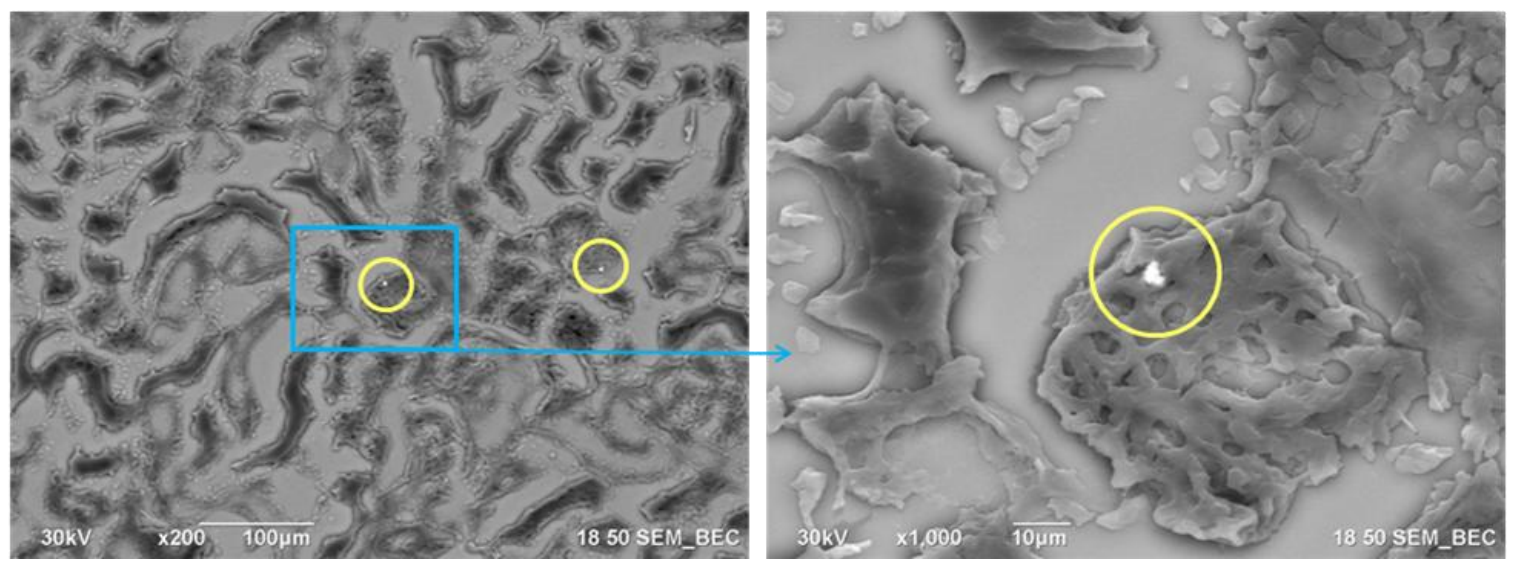

Figure 43. $10 \mathrm{~nm}$ MEN cluster in glomerular region of kidney.

These SEM back-scatter images show an example of MEN clusters in the kidney. The yellow circles highlight the clusters. The image on the right is increased magnification into the blue box to better show the glomerulus. 
The few clusters that were located in the kidneys tended to be centralized in the glomerular regions of the kidney tissue. This finding is not surprising considering that most of the literature supports that only the smallest nanoparticles, typically those that are under $10 \mathrm{~nm}$ and closer to $5 \mathrm{~nm}$, are consistently cleared through the renal clearance pathway [78]-[80]. This is due to the glomerular capillary wall pathway for glomerular filtration, where the filtrate passes through the fenestrated endothelium, glomerular basement membrane, and finally the filtration slits [81]; these filtration slits themselves are approximately $43 \mathrm{~mm}$ wide [82], however the physiological pore size is closer to 5 $\mathrm{nm}$ after the glomerular layer assembly is considered as a collective [83]. In this case, it can be assumed that only the smallest of the synthesized particles, which may fall slightly closer in size to $5 \mathrm{~nm}$, are those that were found in the kidney tissue. Alternatively, any small imperfections in the renal complex tissue could also allow the nanoparticles to enter the glomerular region.

\section{Biodistribution \& Clearance Considerations of the Brain}

MEN clusters found in the brain tissues were very rare and very small. A fully comprehensive brain study can be performed at a later time to determine the exact biodistribution and clearance of different sized MENs through the brain, as the current data is more preliminary in nature. All instances of MENs found in the brain are mapped on the GENSAT Brain Atlas image [84] in Figure 44. The time after the initial MEN administration is noted in Table 8. The instances of particles discovered in this experiment are too sporadic to draw any meaningful conclusions, but the finding of different sized particles in different brain regions throughout different time points is a good starting point for the development of brain cancer specific nanomedicines. 


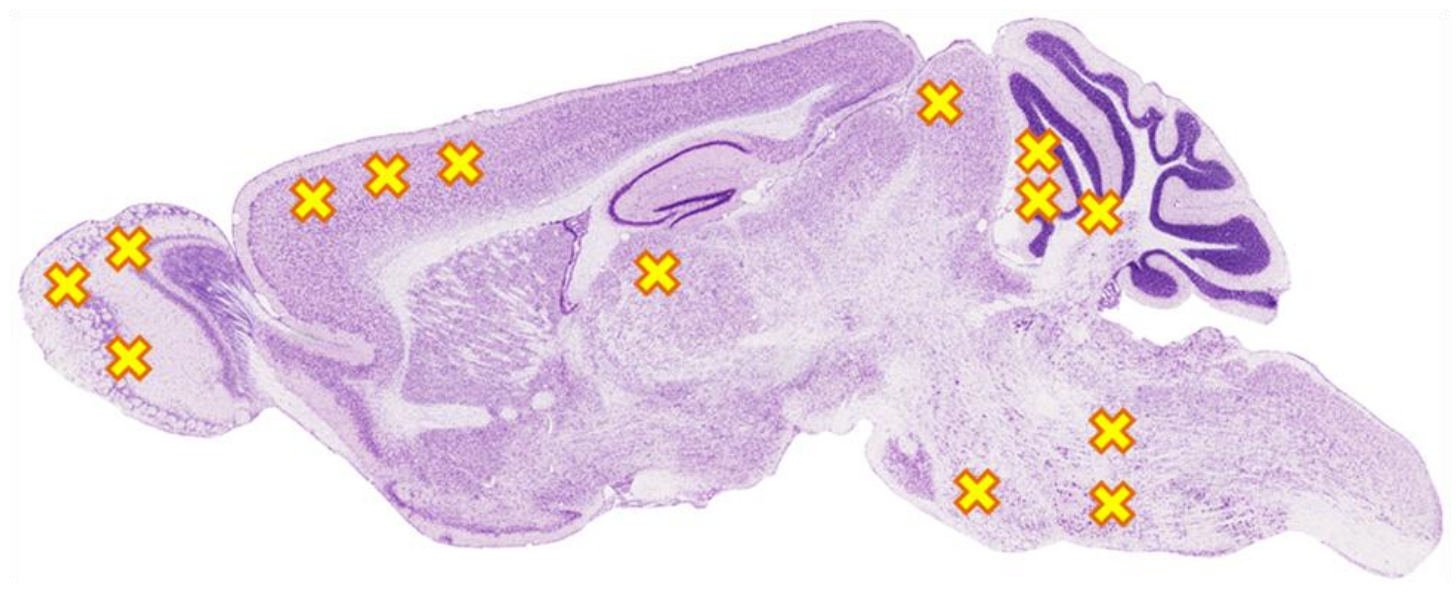

Figure 44. Brain atlas showing located MENs.

All the MEN clusters located in this experiment are approximately displayed on this open access atlas image of a mouse brain.

Table 8. MEN clusters in brain regions over time.

\begin{tabular}{|c|c|c|c|}
\hline & 1 Week & 1 Month & 2 Months \\
\hline Olfactory Bulb & $\checkmark$ & & $\checkmark$ \\
\hline \multicolumn{4}{|l|}{ Cerebral Cortex } \\
\hline Thalamus & $\checkmark$ & & \\
\hline Cerebellum & $\checkmark$ & $\checkmark$ & \\
\hline Midbrain & & & $\checkmark$ \\
\hline Medulla & & $\checkmark$ & $\checkmark$ \\
\hline Pons & & $\checkmark$ & \\
\hline
\end{tabular}

\section{$\underline{6.4 \text { Conclusion }}$}

This chapter has described the biodistribution of MENs in mice receiving cancer treatment, with and without applied magnetic fields, as well as the biodistribution of MENs through the body of healthy mice. Our findings show that our MENs demonstrate strong preference for tumor tissue over peripheral organs, which is further amplified by applying a magnetic field at the site of the tumor for further targeting the MENs into tumor tissue and away from healthy tissue. It is important to note that the goal of the experiments in this chapter was not to quickly discharge the MENs, but quite the 
opposite: to track a large concentration of the MENs over a longer period of time for a more robust understanding of the biodistribution.

Most of the MENs clustered in the liver, spleen, and lungs, as was expected for the size range used [79]. The lung is a very common accumulation point in mouse biodistribution studies for most systemically administered nanoparticles (organic and inorganic) due to the fact that the lung capillary bed is one of the first robustly perfused areas that the nanoparticles encounter after the initiation of circulation from the lateral tail vein IV administration point [85]. Nanoparticles larger than $200 \mathrm{~nm}$ are found to typically accumulate in the liver and spleen, where they are processed for clearance, as well as intermittently in the lungs, however particles smaller than $200 \mathrm{~nm}$ but larger than those filtered through the kidney also tend to follow the same trajectory but to a lesser extent [79]. This explains why our $500 \mathrm{~nm}$ MENs were preferentially concentrated in the lungs, even two months after injection (Figure 42c). The reason behind this sizedependent difference in distribution can better be explained with how nanoparticles leave the bloodstream to enter organ tissues. Specifically, the particles in the size range used here would most likely rely on discontinuous endothelium for their bloodstream exit points [86]. The discontinuous type of endothelium is characteristically found in both the liver and the spleen, and has much larger pores than both continuous endothelium (lining most of the vasculature) and fenestrated endothelium (characteristic of kidney lining). This helps explain why many of the MENs were located in the organs of the reticuloendothelial system (RES) (i.e. liver and the spleen), where they were likely being processed for clearance from the body via RES clearance mechanisms. 
An interesting consideration for the distinctive, albeit small, presence of MENs in the kidney tissue of the cancer mouse group (Figure 39) is that metastasis of SKOV-3 cells into kidney tissue was confirmed in the H\&E staining and HER-2 fluorescent imaging histological studies discussed in Chapter 5 for those animals. The cancer-free biodistribution group having even less identifiable MENs in the kidneys further supports the notion that the MENs were drawn to cancer cells that had metastasized into the kidneys for a slightly stronger presence than in the kidneys of cancer-free mice.

While the brain localization of MENs is still in the preliminary stage regarding the course of localization over time and of the differently sized MENs, we did expect to see MENs present in brain tissue as is documented in this study. This is especially good news for the applicability of MEN based cancer treatment for brain cancers, a particularly challenging cancer type to treat [87]. Likewise, other treatments for brain-related disorders, such as Alzheimer's or Parkinson's, can be fullfulled with MENs as demonstrated in our previous publication [6]; in this previous study, we utilized systemically administered nanoparticles to stimulate the brain with the application of an external magnetic field. The current biodistribution study shows that small amounts of MENs are able to localize into the brain without the assistance of an applied magnetic field. A more elaborate study that shows the exact time, size, and brain region relationships in the biodistribution pattern would be a valuable future venture to undertake. 


\section{CHAPTER 7: DISCUSSION}

\subsection{Study Summary}

The scope of this dissertation covered the advancement of a novel nanoformulation for cancer treatment, MENs, from the knowledge that the treatment is effective in-vitro all the way to mapping the clearance of the treatment from fully cured animal patients in in-vivo studies.

First, we elaborated on the in-vitro mechanics of the treatment. This was done by treating cancer cells with the nanoformulation and an applied magnetic field, removing all solution from the cells, and finally bursting open the cells to locate the MENs inside. With this experiment, we were able to confirm that the MENs enter the cancer cells to deliver the carried drugs. Furthermore, the role of the magnetic field was investigated. We found that an externally applied d.c. magnetic field facilitated the internalization of the MENs into the cancer cell, likely by an electroporation mechanism at the nano scale. After the MENs had entered the cancer cells driven by the d.c. magnetic field, the application of an a.c. magnetic field triggered the release of the carried payload. Therefore, utilizing small, safe, externally controlled magnetic fields, we can trigger the internalization of the whole nanoformulation and later trigger the release of the molecule being delivered to the intracellular space.

Next, we treated a humanized murine model of cancer using the MEN treatment platform. Two different administration routes, three different nanoformulations, and the presence of d.c. and a.c. magnetic fields were all investigated in this initial study. It was determined that a systemic delivery was more effective than a localized delivery for the 
MEN treatment, as more of the cancer cells are exposed to the treatment when it is carried by means of the vasculature as compared to when it simply flushes the periphery of the tumor. Compared to FDA approved organic nanoparticles (PLGA) and to similar magnetic nanoparticles that lack the electric properties of MENs (MNs), MENs were the strongest performing nanomedicine platform. MENs performed similarly with or without additional targeting via an external targeting molecule, while PLGA nanocarriers suffered in performance without these targeting molecules and MNs underperformed in general. The MENs treatment platform was the only platform able to fully cure the mice of their cancer in this study. Further investigation of the MEN platform highlighted the role of the MENs' magneto-electric coupling. While particles that were too small to display the full scope of the magneto-electric coupling (10 nm MENs) remained unaffected, both $30 \mathrm{~nm}$ and the larger $100 \mathrm{~nm}$ MENs improved treatment efficiency with an a.c. magnetic field application following the d.c. magnetic field targeting treatment. This is likely due to the discreet functions of (1) MEN localization using a d.c. magnetic field application and (2) MEN-carried payload release using an a.c. magnetic field application, as were demonstrated in the in-vitro study previously.

Finally, we wanted to establish an understanding of the biodistribution and clearance of the MENs in the mice. We compared the mice from the cancer group treated over time to healthy mice which received a single high dose of the MENs and were mapped for nanoparticle distribution at varying time points post-injection. The cancer mouse group further highlighted the role of the applied magnetic field in the cancer treatment, as mice without any magnetic fields to induce the internalization of the treatment into the tumor had notably less MENs in the tumor and more in the periphery; 
on the other hand, magnetic field treated mice had the largest portion of their MEN biodistribution localized in the tumor tissue. Mice successfully cured of their cancer had very little MENs remaining in their body after a three-month recovery period. The clearance times and varying MEN sizes revealed the following:

1) $10 \mathrm{~nm}$ MENs clear the most rapidly, likely able to utilize some extent of renal clearance. $500 \mathrm{~nm}$ MENs had the lowest initial retention, however these large MENs were also the most numerous at the furthest time point in the study.

2) The lung showed the highest initial retention of any peripheral organ, followed by transition into RES organs (i.e. the liver and the spleen) by all sizes. The RES is likely responsible for most of the MEN clearance.

3) Very small amounts of the MENs were found in kidney and brain tissues throughout the entire study.

Overall, these studies have shown that the previously reported efficacy of the MEN cancer treatment platform does indeed translate from in-vitro to in-vivo. Furthermore, the MEN drug delivery platform, which uniquely relies only on general physical properties, is the most effective of its kind, and the biodistribution and clearance patterns of this nanocarrier suggest clearance primarily through the reticuloendothelial system.

\section{$\underline{7.2 \text { Discussion }}$}

\subsubsection{The MEN Targeting Mechanism}

One of the unique features of MENs is their ability to be influenced externally using spatial magnetic field gradients after administration to assist in guiding the particles 
to the target site; this feature is shared with other types of strong magnetic nanoparticles as well. Non-magnetic nanoparticles that rely on either (a) the passive EPR effect, (b) active targeting by means of surface antibodies or ligands, or even (c) a combination of both are still limited in targeting potential in that they cannot be assisted towards the target site post-administration. The establishment of near perfect targeting capabilities is the goal of essentially any drug delivery platform, and magnetic field directed targeting is an additional feature held by magnetic nanoparticles that complements their inherent EPR and active targeting abilities. Active targeting molecules must be highly specific for different types of cancer, making this type of targeting less generalizable than the passive EPR and magnetic gradient targeting mechanisms. In a push for an adaptable cancer treatment, the physical accumulation of small molecules (i.e. the nanoformulation) by the EPR effect can be enhanced by further pooling the magnetic nanoparticle carried treatment at the general site of the tumor. With the magnetic nanoformulation being delayed in the vicinity of the tumor by means of an external magnetic field, the vasculature of the tumor has a greater opportunity to "leak" the nanoformulation into the tumor tissues by means of EPR. This more generalized (at least to solid tumors that establish an EPR effect) magnetic nanoparticle based treatment can then be further enhanced with active targeting molecules for greater specificity to individual cancer types.

MENs alone offer one additional targeting mechanism, which is not shared with any other nanoparticle based cancer treatment, and that is targeting by means of electric fields. Due to the varying cell membrane permeability to ions (such as $\mathrm{Na}^{+}, \mathrm{K}^{+}, \mathrm{Ca}^{2+}$, and $\mathrm{Cl}^{-}$) that causes an uneven distribution of the ions, a voltage difference is established 
between the intracellular and extracellular environment; this voltage (relative to the extracellular environment) is the membrane potential, and cancer cells are known to have a partially depolarized membrane potential favoring cell proliferation [62], [88]. Since the cell membrane is an electrically polarizable medium, and cancer cells have distinctive electrophysiological properties, MENs can target cancer cells in a novel physical method that relies on the differences in these electrophysiological properties.

An intravenously administered MEN traveling through the circulatory solution (either blood or lymph) expresses its negative electric charge generated by means of the double layer colloidal chemistry on the MEN surface (zeta potential). This charge, combined with the smaller electric charge generated by the magneto-electric dipole, is amplified by an external magnetic field proportionally to the applied field strength, and allows the MENs to interface preferentially with the membranes of cancer cells. Therefore, an externally applied magnetic field creates a two-part cancer targeting mechanism for the MENs, as shown Figure 45. The first targeting mechanism is the simple magnetic pull of the MENs towards the tumor site, where higher chances of MEN-cancer cell interaction (leading to cellular internalization of the nanoformulation) is improved through increased MEN availability by means of accumulation at the vicinity of the tumor. The second targeting mechanism results from the MENs' non-zero magneto-electric coefficient and is the amplification of the negative surface charge on the MENs by the externally applied magnetic field. In slightly alkaline cellular environments, like blood, cell membranes are typically exhibiting a negative electric charge [89]. This is advantageous to the MENs, as it establishes a negative-negative repulsion force that allows easier passage through the capillaries without being trapped by surrounding cells. 
Considering that cancer cells have less negative membrane potentials compared to their healthy counterparts, the repulsion forces between MENs and cancer cells are weaker than the repulsion forces between MENs and healthy cells. Furthermore, when a MEN reaches the exterior of a cancer cell (through EPR, active targeting, and/or magnetic pull), the MEN's surface charge can generate an electric field on the order of $1000 \mathrm{~V} / \mathrm{cm}$ to create a localized nanoelectroporation effect. This nano-scale electroporation causes the cell membrane to locally rearrange the phospholipid bilayer at the immediate site of the nanoparticle, allowing the nanoparticle entry directly through the membrane. Due to the inherent differences in cell membrane properties between cancer cells and healthy cells, the electric field required to electroporate healthy cells is significantly higher (closer to the range of 5000-10,000 V/cm) [90], [91]. This difference in cellular electric properties allows healthy cells to be spared from MEN nanoelectroporation.

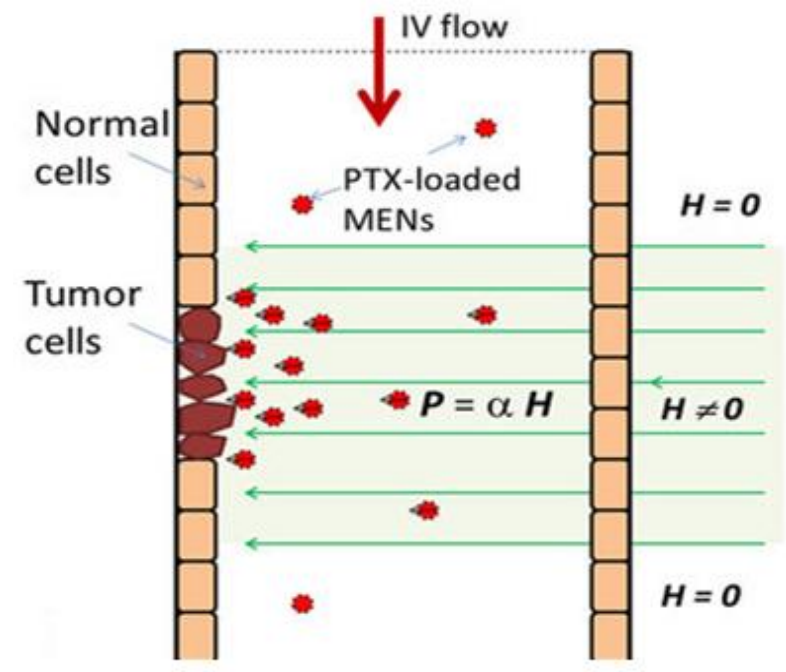

Figure 45. MEN cancer targeting in circulation.

Drug-loaded MENs traveling through the circulation are drawn to cancer cells with magnetic and electric fields. An applied magnetic field, $\mathrm{H}$, pushes the MENs towards the site of the cancer. Additionally, the non-zero magneto-electric coefficient, $\alpha$, influenced by $\mathrm{H}$, polarizes the electric dipole, $\mathrm{P}$, and increases the strength of the negative surface charge of the MEN. This creates a localized attraction force based on electric conductivity between the MENs and the cancer cells that is not shared by healthy cells. 
This hypothesis for in-vivo physical targeting is supported by the mouse trials, where we found that MNs were not able to readily localize into the tumor tissue despite their $40 \mathrm{x}$ higher magnetization potential. This illustrates the importance of the magnetoelectric effect and surface charge manipulation by external magnetic fields in order to internalize the nanoformulation into the cancer cells. While the MNs were similarly drawn to the tumor with the externally applied magnetic field, it seems that the MNs were less capable of intracellular entry because of a lack of the nanoelectroporation effect; the MNs likely relied on the EPR effect. This also helps demonstrate that the magnetoelectric coupling of MENs grants a separate form of targeting that is complemented by, but not reliant on, the EPR effect. The electric field based discrimination of cancer cells by MENs allows this treatment to be applicable to cancers that lack a large, solid tumor with an established EPR effect, such as early-stage cancers or the metastasized cells of later-stage cancers.

\subsubsection{The MEN Payload Release Mechanism}

Another unique advantage of the MEN platform is the capability to externally release the carried payload on demand. One of the primary challenges facing drug delivery platforms is a reliable release, one that is not premature and incomplete. The inherent properties of cancer physiology (i.e. the tumor microenvironment) offer some drug release options that are often utilized in nanomedicine [21]. Specifically, local tumor $\mathrm{pH}$ [92], [93] and tumor-prevalent enzymes [94], [95] are popular mechanisms for releasing drugs from nanocarriers. Drug release platforms relying on the minute differences of the tumor microenvironment suffer from non-ideal specificity, as the same 
conditions can exist in normal physiological conditions (even if to a lesser extent) and are often too similar in parameters to reliably distinguish. For this reason, externally triggered drug release platforms are gaining favor, often in the form of heat generation [96] or ultrasound [97] drug release. Ultrasound drug release treatments tend to be utilized mostly for organic nanoparticle platforms. Temperature-reliant platforms in general tend to suffer from non-uniform heating, and any internal generation of heat has the potential to cause collateral damage to nearby healthy tissues. Our MEN platform offers a new externally controlled payload release mechanism based on magnetic field manipulation.

Figure 46 summarizes the entire multistep process of the MENs cancer treatment as pertaining to the external magnetic field manipulation. When no field is applied (Figure 46a), few MENs are able to internalize into cancer cells, although the small baseline magnetization of the MENs as well as passive EPR will likely allow a small portion of the treatment to internalize. In order for the MENs to nanoelectroporate and readily enter the cell, an external magnetic field which surpasses the electroporation threshold of the cell membrane must be applied (Figure 46b). Again, it is important to stress that this electroporation threshold will be dramatically smaller for cancer cells than for healthy cells. The final step of payload release is then initiated by increasing the strength of the d.c. magnetic field being applied or by initiating an a.c. magnetic field application (Figure 46c). Applying magnetic fields to magnetic particles has the potential to generate heat, however Figure 47 shows the complete absence of heat generation in MENs throughout the entire field-exposure process. 


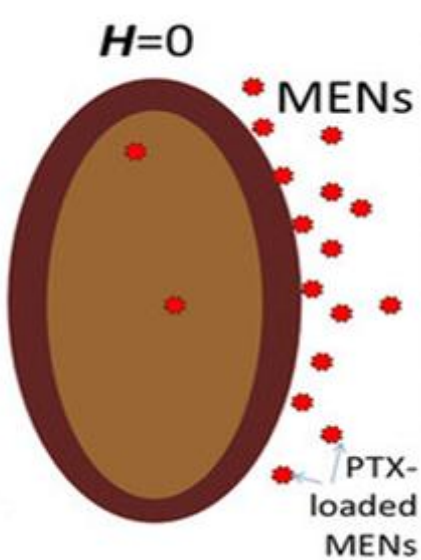

a)

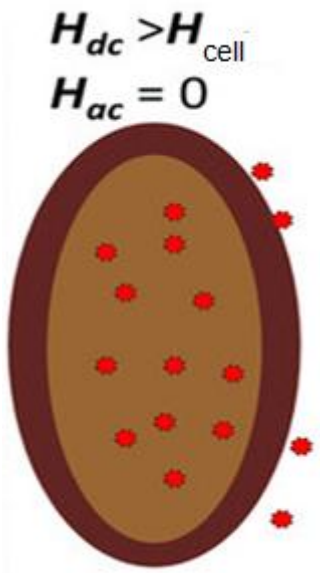

b)

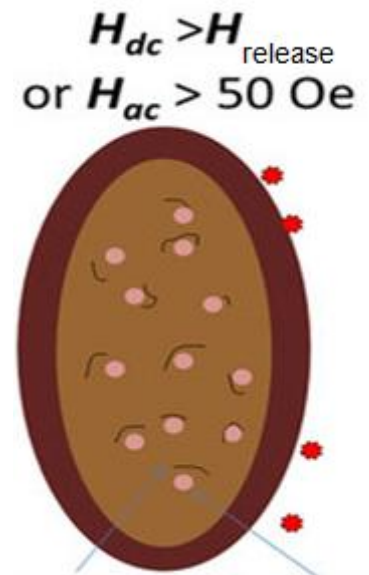

c)
PTX unloaded off MENs

Figure 46. Magnetic fields control MEN cellular entry and drug release.

(a) When no field is present ( $\mathrm{H}=0)$, very few MENs internalize into cells. (b) When the d.c. field is raised beyond the nanoeletroporation threshold field for the cancer cells $\left(\mathrm{H}_{\text {cell }}\right)$, the MENs readily cross the cell membrane and internalize. (c) When the MENs-drug bond release threshold $\left(\mathrm{H}_{\text {release }}\right)$ is surpassed, either by means of a stronger d.c. field or an a.c. field, the payload is released inside the cell.
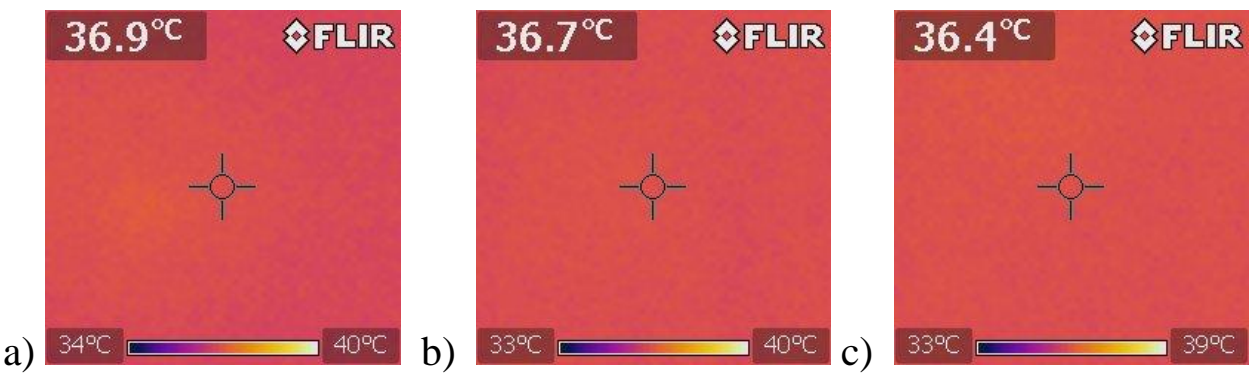

Figure 47. MENs generate no heat with applied fields.

Cells incubated with MENs with (a) no field applied (b) 12 hour d.c. field application (c) 12 hour a.c. field application following 12 hour d.c. field application. No detectable changes in heat are observed.

An a.c. field of approximately $50 \mathrm{Oe}$ at $100 \mathrm{~Hz}$ is sufficient to release the PTX payload from the GMO coated MENs used in this series of experiments. This field strength is still far too weak to cause any notable interaction of MENs with healthy cells. The exact release threshold value will likely vary for different treatments loaded onto different functional coatings, but any MEN nanoformulation can be successfully delivered and the payload released with a correct combination of functional coating and applied magnetic field (Chapter 3.4). As long as the threshold to release the carried drug 
is substantially higher than the threshold to enter the targeted cell $\left(\mathrm{H}_{\text {release }}>\mathrm{H}_{\text {cell }}\right)$, the nanoformulation is viable for externally controlled targeting and payload release. Both $\mathrm{H}_{\text {release }}$ and $\mathrm{H}_{\text {cell }}$ must remain below the nanoeletroporation threshold field for healthy cells for healthy tissue to remain unharmed by the treatment.

\subsubsection{The MEN Clearance Mechanism}

We have found that our MENs appear to be primarily utilizing the RES (i.e. liver and spleen) for biological clearance, as deducted from robust presence in liver and spleen tissue and minimal presence in kidney tissue. This is consistent with the pore sizes associated with these organs. Renal clearance is such a fast and efficient process that evasion of renal clearance is actually a very valuable feature. Nanoparticles too large for renal clearance are able to remain in the blood plasma significantly longer than smaller molecules, and this is a critical component of the EPR effect [98]. The RES clears larger sized nanoparticles by means of Kupffer cells and macrophages [85]. Nanoparticles larger than $100 \mathrm{~nm}$ are reported to activate the classical complement pathway and macrophage internalization, both in a size dependent manner, with larger particles being recognized and cleared the fastest; however, the exact nanoparticle formulation as well as the surface charge also affects the rate of the mononuclear phagocyte clearance [99], [100]. Therefore, nanoparticles larger than $10 \mathrm{~nm}$ but smaller than $100 \mathrm{~nm}$ are considered to be in the ideal size range to evade both renal and RES clearance mechanisms long enough to benefit from EPR enhanced delivery. This is likely why our $30 \mathrm{~nm}$ MENs performed better than $10 \mathrm{~nm}$ and $100 \mathrm{~nm}$ sized MENs, both of which bordered on the size range required for renal and RES clearance, respectively. 
Particles sized at approximately $500 \mathrm{~nm}$ and larger are not considered ideal for cancer treatment because their large sizes hinders their ability to benefit from the EPR effect in two ways: (1) the large size makes them candidates for rapid clearance from the body, not giving them enough time to localize into the tumor and (2) the leaky vasculature of the tumors more readily passes smaller nanoparticles into the internal tumor tissue. However, a larger size allows for potentially stronger physical properties (especially in the form of magnetics) to be displayed and for more elaborate payloads and targeting molecules to be loaded onto the surface. Therefore, particles of this size still merit investigation, as surface functionalizations can help alleviate rapid clearance issues and some tissues and tissue cancers have larger pores that can accept entry of molecules of this size. To eliminate a potentially strong magnetizer for the larger size seems premature in this case. The clearance by the macrophages of the RES is influenced by the protein absorption and opsonization of the nanoparticles, which is determined by physical attributes such as the particle's surface chemical composition, surface charge, and hydrophobic/hydrophilic properties, as well as overall particle size and shape; every one of these properties can be modified, however, particles exceeding the nano-scale and entering the macro-scale mimic the size of bacteria and are cleared by macrophages with very high efficiency [99], [101], [102]. MENs can also be modified in surface properties, size, and shape to establish the desired effect while maintaining the desired biodistribution and clearance patterns.

Inorganic or non-biodegradable nanoparticles tend to spark concern for long term retention. However, as long as there are no toxicity issues, long term nanoparticle bioretention is not necessarily a bad thing, as one of the primary principles of nanoparticle 
drug delivery is the increase of the circulation time of the drug. Exceedingly rapid clearance of nanoparticles would hinder such goals. Any sort of longer term slow-release nanoformulation would absolutely benefit from clearance evasion and longer circulation and retention durations. MENs are a good candidate for slow-release nanoformulations for two primary reasons: (1) MENs effectively evade rapid clearance, as shown in Chapter 6, and (2) MENs are externally triggered to release their carried payload, as shown in Chapter 4. By adjusting the strength of the payload-releasing a.c. magnetic field, the release pattern can be slowed down to a trickle model instead of a burst model. Furthermore, a combination of functionalizations, such as those investigated in [64], can be utilized to establish MENs of different payload release strengths and combined into one long-term treatment. This slow-release MEN treatment would be composed of the differently functionalized MENs that are administered simultaneously, and targeted for internalization with a d.c. magnetic field simultaneously, but can then be activated to release their carried drugs at multiple different times by using different a.c. fields of gradually increasing strengths.

\subsubsection{MENs as an All-in-One Cancer Therapy}

We have demonstrated here that our novel MEN cancer therapy is not only effective in-vivo, but holds many advantages over other nanoparticle based therapies. One such advantage is the multifaceted approach it can provide to patient care, as summarized in Table 9. MENs provide a reliable drug delivery and drug release platform that can simultaneously be used to monitor patient progress. 
Table 9. MEN multifaceted cancer therapy.

\begin{tabular}{|c|c|c|}
\hline Cancer Targeting & Drug Release & Imaging \\
\cline { 1 - 2 } Electric charge & \multirow{2}{*}{ External DC Field } & Conventional MRI \\
\cline { 1 - 2 } Magnetic pull & \multirow{2}{*}{ External AC Field } & \multirow{2}{*}{ High Resolution MNI } \\
\cline { 1 - 2 } Passive (EPR) &
\end{tabular}

Furthermore, there is still room for customization in both the targeting and payload release parameters of the MENs. As shown in Chapter 5, MENs function with or without additional targeting molecules, and further targeting can be established with the addition of surface molecules as needed. Furthermore, as seen in the comparison of MENs to non-magnetoeletric MNs of the same size, it seems that MENs use a mechanism distinctive from EPR to reach the cancer cells, making the MENs treatment applicable beyond large, fixed tumor masses. Overall, MENs have the ability to utilize conventional nanoparticle targeting approaches while also boasting their unique electric field based cancer discrimination. The drug loading, and therefore the drug release, mechanism also has room for tailoring the platform to different drug formulations. As discussed in Chapter 3.4, different functional coatings can be utilized to provide distinctive surface properties and varying bond strengths with the payload. Depending on the bond strength between the MENs and the loaded drug, the magnetic field used to break that bond $\left(\mathrm{H}_{\text {release }}\right)$ can be adjusted accordingly. The MEN-payload bond can be broken using a stronger d.c. magnetic field than that used for the targeting $\left(\mathrm{H}_{\text {release }}>\mathrm{H}_{\text {cell }}\right)$ or an a.c. magnetic field, which provides further options for customizing the nanoformulation. 
Lastly, MENs, like conventional magnetic nanoparticles, can safely be imaged using conventional MRI technology [54], [103]-[105], as well as newer, high-resolution, 3D real-time magnetic nanoparticle imaging technology [56]. The implication for patient care is that the MENs can both deliver the desired treatment to the cancer and simultaneously serve as the contrast agent for imaging technology for live monitoring of treatment efficacy. For cancer treatment, MENs offer an unparalleled potential:

1) Generally applicability to any cancer

2) Multiple drug payload possibilities

3) High specificity cancer discrimination

4) Reliable externally controlled drug release

5) Contrast agent capability

6) General potential for further modifications for further specialization

7) Overall a highly personalizable treatment that can be tailored to each patient For these reasons, we believe that the MENs platform has successfully answered the call to action by the national "Moonshot" initiative and has immense potential to be widely utilized in medical practice in the future.

\section{$\underline{7.3 \text { Future Studies }}$}

This study established a general efficacy of this cancer treatment in-vivo as well as characterized the general biodistribution and clearance properties of the nanocarrier for the very first time. One of the obvious limitations is the small sample size. However, in consideration of maintaining the highest standard of animal research ethics, this study was deliberately kept small at this stage. The overwhelming success demonstrated by this 
small pilot study warrants further investigation with larger cohorts to establish more precise pharmacokinetics, biodistribution, and clearance rates in general. Specifically, exact dose dependence should be established. It would also be interesting to compare cancer-treated mice to cancer-free mice for biodistribution with identical progressive dosages of MENs. More animals dedicated to the cancer treatment experiment would be particularly valuable as there was notable heterogeneity of disease progression from the xenografts, which truthfully mimics the medical reality of human cancer patient diversity.

Furthermore, the small size of nanoparticles grants them entry past the BBB and into the brain, making the use of nanoparticles for drug delivery to the brain a popular application of nanomedicine [106]. We would like to continue investigating the brain targeting potential of MENs first reported in a previous publication [6]. We have initiated a behavioral study component to the current study. While the mice used in this study appear to be completely unaffected by the MEN treatment (as determined by behavioral observation), a comprehensive, maze-based memory analysis will confirm any changes in cognition. It will also allow us to more accurately map the biodistribution of MENs throughout the brain.

Finally, the treatment can be scaled up and investigated in a larger animal. The ultimate goal would be to progress this treatment into clinical trials, so any steps that need to be taken towards this goal are recommended as a future course of action. 


\section{REFERENCES}

[1] NCI, “Cancer Statistics,” NIH. [Online]. Available: http://www.cancer.gov/aboutcancer/what-is-cancer/statistics.

[2] SEER, "SEER Stat Fact Sheets: All Cancer Sites," NIH. [Online]. Available: http://seer.cancer.gov/statfacts/html/all.html.

[3] The White House, "Investing in the National Cancer Moonshot," Off. Press Secr., 2016.

[4] F. J. Heiligtag and M. Niederberger, "The fascinating world of nanoparticle research," Mater. Today, vol. 16, no. 7-8, pp. 262-271, 2013.

[5] K. Yue, R. Guduru, J. Hong, P. Liang, M. Nair, and S. Khizroev, "MagnetoElectric Nano-Particles for Non-Invasive Brain Stimulation," PLoS One, vol. 7, no. 9, p. e44040, Sep. 2012.

[6] R. Guduru, P. Liang, J. Hong, A. Rodzinski, A. Hadjikhani, J. Horstmyer, E. Levister, and S. Khizroev, "Magnetoelectric 'spin' on stimulating the brain," Nanomedicine, vol. 10, no. 13, pp. 2051-2061, Jul. 2015.

[7] A. B. Ryerson, C. R. Eheman, S. F. Altekruse, J. W. Ward, A. Jemal, R. L. Sherman, S. J. Henley, D. Holtzman, A. Lake, A.-M. Noone, R. N. Anderson, J. Ma, K. N. Ly, K. A. Cronin, L. Penberthy, and B. A. Kohler, "Annual Report to the Nation on the Status of Cancer, 1975-2012, featuring the increasing incidence of liver cancer," Cancer, vol. 122, no. 9, pp. 1312-1337, May 2016.

[8] B. A. Chabner and T. G. Roberts, "Chemotherapy and the war on cancer," Nat Rev Cancer, vol. 5, no. 1, pp. 65-72, Jan. 2005.

[9] V. T. DeVita and E. Chu, "A history of cancer chemotherapy," Cancer Res., vol. 68, no. 21, pp. 8643-8653, 2008.

[10] R. Baskar, K. Ann-Lee, R. Yeo, and K.-W. Yeoh, "Cancer and Radiation Therapy: Current Advances and Future Directions," Int. J. Med. Sci., vol. 9, no. 3, pp. 193199, 2012.

[11] S. C. Formenti and S. Demaria, "Systemic effects of local radiotherapy," Lancet Oncol., vol. 10, no. 7, pp. 718-726, Jul. 2009.

[12] A. C. Begg, F. A. Stewart, and C. Vens, "Strategies to improve radiotherapy with targeted drugs," Nat Rev Cancer, vol. 11, no. 4, pp. 239-253, Apr. 2011. 
[13] NCI, “Targeted Therapy,” NIH. [Online]. Available:

http://www.cancernet.nci.nih.gov/about-cancer/treatment/types/targeted-therapies.

[14] D. E. Gerber, "Targeted therapies: a new generation of cancer treatments.," Am. Fam. Physician, vol. 77, no. 3, pp. 311-9, Feb. 2008.

[15] M. Vanneman and G. Dranoff, "Combining immunotherapy and targeted therapies in cancer treatment," Nat. Rev. Cancer, vol. 12, no. 4, pp. 237-251, Mar. 2012.

[16] V. V. Padma, "An overview of targeted cancer therapy.," BioMedicine, vol. 5, no. 4, p. 19, Nov. 2015.

[17] N. Jiang, X. Wang, and Y. Y. and W. Dai, "Advances in Mitotic Inhibitors for Cancer Treatment," Mini-Reviews in Medicinal Chemistry, vol. 6, no. 8. pp. 885895, 2006.

[18] K.-S. Chan, C.-G. Koh, and H.-Y. Li, "Mitosis-targeted anti-cancer therapies: where they stand," Cell Death Dis., vol. 3, no. 10, p. e411, 2012.

[19] K. E. Gascoigne and S. S. Taylor, "How do anti-mitotic drugs kill cancer cells?," J. Cell Sci., vol. 122, no. 15, pp. 2579-2585, 2009.

[20] N. Nishida, H. Yano, T. Nishida, T. Kamura, and M. Kojiro, "Angiogenesis in cancer," Vasc. Health Risk Manag., vol. 2, no. 3, pp. 213-219, 2006.

[21] L. Brannon-Peppas and J. O. Blanchette, "Nanoparticle and targeted systems for cancer therapy," Adv. Drug Deliv. Rev., vol. 64, pp. 206-212, 2012.

[22] Z. Wang, C. Dabrosin, X. Yin, M. M. Fuster, A. Arreola, W. K. Rathmell, D. Generali, G. P. Nagaraju, B. El-Rayes, D. Ribatti, Y. C. Chen, K. Honoki, H. Fujii, A. G. Georgakilas, S. Nowsheen, A. Amedei, E. Niccolai, A. Amin, S. S. Ashraf, B. Helferich, X. Yang, G. Guha, D. Bhakta, M. R. Ciriolo, K. Aquilano, S. Chen, D. Halicka, S. I. Mohammed, A. S. Azmi, A. Bilsland, W. N. Keith, and L. D. Jensen, "Broad targeting of angiogenesis for cancer prevention and therapy," Semin. Cancer Biol., vol. 35, pp. S224-S243, 2015.

[23] A. Z. Wang, R. Langer, and O. C. Farokhzad, "Nanoparticle Delivery of Cancer Drugs," Аnпи. Rev. Med., vol. 63, no. 1, pp. 185-198, 2012.

[24] R. K. Jain and T. Stylianopoulos, "Delivering nanomedicine to solid tumors.," Nat. Rev. Clin. Oncol., vol. 7, no. 11, pp. 653-664, Nov. 2010.

[25] H. Maeda, J. Wu, T. Sawa, Y. Matsumura, and K. Hori, "Tumor vascular permeability and the EPR effect in macromolecular therapeutics: A review," $J$. Control. Release, vol. 65, no. 1-2, pp. 271-284, 2000. 
[26] H. Kobayashi, R. Watanabe, and P. L. Choyke, "Improving conventional enhanced permeability and retention (EPR) effects; What is the appropriate target?," Theranostics, vol. 4, no. 1, pp. 81-89, 2014.

[27] A. M. Scott, J. P. Allison, and J. D. Wolchok, "Monoclonal antibodies in cancer therapy," Cancer Immun., vol. 12, no. 14, pp. 1-8, 2012.

[28] R. V. J. Chari, "Targeted cancer therapy: Conferring specificity to cytotoxic drugs," Acc. Chem. Res., vol. 41, no. 1, pp. 98-107, 2008.

[29] P. Sharma and J. P. Allison, "Immune checkpoint targeting in cancer therapy: Toward combination strategies with curative potential," Cell, vol. 161, no. 2, pp. 205-214, 2015.

[30] N. Bertrand, J. Wu, X. Xu, N. Kamaly, and O. C. Farokhzad, "Cancer nanotechnology: The impact of passive and active targeting in the era of modern cancer biology," Adv. Drug Deliv. Rev., vol. 66, pp. 2-25, 2014.

[31] F. Alexis, E. M. Pridgen, R. Langer, and O. C. Farokhzad, Drug Delivery, vol. 197. 2010.

[32] V. P. Torchilin, "Recent advances with liposomes as pharmaceutical carriers.," Nat. Rev. Drug Discov., vol. 4, no. 2, pp. 145-160, 2005.

[33] K. S. Soppimath, T. M. Aminabhavi, A. R. Kulkarni, and W. E. Rudzinski, "Biodegradable polymeric nanoparticles as drug delivery devices," J. Control. Release, vol. 70, no. 1, pp. 1-20, 2001.

[34] F. Gu, L. Zhang, B. a. Teply, N. Mann, a. Wang, a. F. Radovic-Moreno, R. Langer, and O. C. Farokhzad, "Precise engineering of targeted nanoparticles by using self-assembled biointegrated block copolymers," Proc. Natl. Acad. Sci., vol. 105, no. 7, pp. 2586-2591, 2008.

[35] Z. L. Tyrrell, Y. Shen, and M. Radosz, "Fabrication of micellar nanoparticles for drug delivery through the self-assembly of block copolymers," Prog. Polym. Sci., vol. 35, no. 9, pp. 1128-1143, 2010.

[36] G. A. Husseini and W. G. Pitt, "Micelles and nanoparticles for ultrasonic drug and gene delivery," Adv. Drug Deliv. Rev., vol. 60, no. 10, pp. 1137-1152, Jun. 2008.

[37] W. Lohcharoenkal, L. Wang, Y. C. Chen, and Y. Rojanasakul, "Protein nanoparticles as drug delivery carriers for cancer therapy," Biomed Res. Int., vol. 2014, 2014. 
[38] H. Sharma, P. K. Mishra, S. Talegaonkar, and B. Vaidya, "Metal nanoparticles: a theranostic nanotool against cancer.," Drug Discov. Today, vol. 20, no. 9, pp. 1143-1151, May 2015.

[39] M. Vinardell and M. Mitjans, "Antitumor Activities of Metal Oxide Nanoparticles," Nanomaterials, vol. 5, no. 2, pp. 1004-1021, 2015.

[40] I. I. Slowing, J. L. Vivero-Escoto, C.-W. Wu, and V. S.-Y. Lin, "Mesoporous silica nanoparticles as controlled release drug delivery and gene transfection carriers," Adv. Drug Deliv. Rev., vol. 60, no. 11, pp. 1278-1288, 2008.

[41] Y. Wang, Q. Zhao, N. Han, L. Bai, J. Li, J. Liu, E. Che, L. Hu, Q. Zhang, T. Jiang, and S. Wang, "Mesoporous silica nanoparticles in drug delivery and biomedical applications," Nanomedicine Nanotechnology, Biol. Med., vol. 11, no. 2, pp. 313$327,2015$.

[42] R. Probst, J. Lin, A. Komaee, A. Nacev, Z. Cummins, and B. Shapiro, "Planar steering of a single ferrofluid drop by optimal minimum power dynamic feedback control of four electromagnets at a distance," J. Magn. Magn. Mater., vol. 323, no. 7, pp. 885-896, Apr. 2011.

[43] M. Arruebo, R. Fernandez-Pacheco, M. R. Ibarra, and J. Santamaria, "Magnetic nanoparticles for drug delivery," Nano Today, vol. 2, no. 3, pp. 22-32, 2007.

[44] J. L. Kirschvink, M. M. Walker, and C. E. Diebel, "Magnetite-based magnetoreception," Current Opinion in Neurobiology. 2001.

[45] D. J. Dunlop, "Superparamagnetic and single-domain threshold sizes in magnetite," J. Geophys. Res., vol. 78, no. 11, pp. 1780-1794, 1973.

[46] Z. Li, L. Wei, M. Y. Gao, and H. Lei, "One-Pot Reaction to Synthesize Biocompatible Magnetite Nanoparticles," Adv. Mater., vol. 17, no. 8, pp. 10011005, Apr. 2005.

[47] T. Neuberger, B. Schöpf, H. Hofmann, M. Hofmann, and B. von Rechenberg, "Superparamagnetic nanoparticles for biomedical applications: Possibilities and limitations of a new drug delivery system," J. Magn. Magn. Mater., vol. 293, no. 1, pp. 483-496, 2005.

[48] P. J. Hoopes, "NIH Public Access,” vol. 1, pp. 1-23, 2013.

[49] A. E. Deatsch and B. A. Evans, "Heating efficiency in magnetic nanoparticle hyperthermia," J. Magn. Magn. Mater., vol. 354, pp. 163-172, 2014. 
[50] R. K. Gilchrist, B. Medal, W. D. Shorey, R. C. Hanselman, J. C. Parrott, and C. B. Taylor, "Selective Inductive Heating of Lymph Nodes," Ann. Surg., vol. 146, no. 4, pp. 596-606, 1957.

[51] D. K. Chatterjee, P. Diagaradjane, and S. Krishnan, "Nanoparticle-mediated hyperthermia in cancer therapy," Ther. Deliv., vol. 2, no. 8, pp. 1001-1014, 2011.

[52] R. Hergt, S. Dutz, R. Müller, and M. Zeisberger, "Magnetic particle hyperthermia: nanoparticle magnetism and materials development for cancer therapy," J. Phys. Condens. Matter, vol. 18, no. 38, pp. S2919-S2934, Sep. 2006.

[53] A. Singh and S. K. Sahoo, "Magnetic nanoparticles: a novel platform for cancer theranostics.," Drug Discov. Today, vol. 19, no. 4, pp. 474-81, Apr. 2014.

[54] D. K. Kim, Y. Zhang, J. Kehr, T. Klason, B. Bjelke, and M. Muhammed, "Characterization and MRI study of surfactant-coated superparamagnetic nanoparticles administered into the rat brain," J. Magn. Magn. Mater., vol. 225, no. 1-2, pp. 256-261, 2001.

[55] B. Gleich and J. Weizenecker, "Tomographic imaging using the nonlinear response of magnetic particles.," Nature, vol. 435, no. 7046, pp. 1214-7, Jun. 2005.

[56] J. Weizenecker, B. Gleich, J. Rahmer, H. Dahnke, and J. Borgert, "Threedimensional real-time in vivo magnetic particle imaging," Phys. Med. Biol., vol. 54, no. 5, pp. L1-L10, 2009.

[57] S. Xie, F. Ma, Y. Liu, and J. Li, "Multiferroic CoFe2O4-Pb( $\operatorname{Zr}(0.52) \mathrm{Ti}(0.48)) \mathrm{O} 3$ core-shell nanofibers and their magnetoelectric coupling.," Nanoscale, vol. 3, no. 8, pp. 3152-8, 2011.

[58] W. Eerenstein, N. D. Mathur, and J. F. Scott, "Multiferroic and magnetoelectric materials.," Nature, vol. 442, no. 7104, pp. 759-65, 2006.

[59] M. Nair, R. Guduru, P. Liang, J. Hong, V. Sagar, and S. Khizroev, "Externally controlled on-demand release of anti-HIV drug using magneto-electric nanoparticles as carriers," Nat. Commun., vol. 4, p. 1707, 2013.

[60] R. Guduru, P. Liang, C. Runowicz, M. Nair, V. Atluri, and S. Khizroev, "Magneto-electric Nanoparticles to Enable Field-controlled High-Specificity Drug Delivery to Eradicate Ovarian Cancer Cells," Sci. Rep., vol. 3, p. 2953, Oct. 2013.

[61] A. Rodzinski, R. Guduru, P. Liang, A. Hadjikhani, T. Stewart, E. Stimphil, C. Runowicz, R. Cote, N. Altman, and R. Datar, "Targeted and controlled anticancer 
drug delivery and release with magnetoelectric nanoparticles," Sci. Rep., vol. 6, no. 20867, pp. 1-14, 2016.

[62] M. Yang and W. J. Brackenbury, "Membrane potential and cancer progression," Front. Physiol., vol. 4 JUL, no. July, pp. 1-10, 2013.

[63] H. Qiu, R. P. Joshi, and A. Pradhan, "Simulation of nanoparticle based enhancement of cellular electroporation for biomedical applications," J. Appl. Phys., vol. 116, no. 18, 2014.

[64] R. Guduru and S. Khizroev, "Magnetic Field-Controlled Release of Paclitaxel Drug from Functionalized Magnetoelectric Nanoparticles," Part. Part. Syst. Charact., vol. 31, no. 5, pp. 605-611, 2014.

[65] G. Pillai, "Nanomedicines for Cancer Therapy: An Update of FDA Approved and Those under Various Stages of Development," SOJ Pharm Pharm Sci, vol. 1, no. 2, p. 13, 2014.

[66] S. Kunjachan, R. Pola, F. Gremse, B. Theek, J. Ehling, D. Moeckel, B. HermannsSachweh, M. Pechar, K. Ulbrich, W. E. Hennink, G. Storm, W. Lederle, F. Kiessling, and T. Lammers, "Passive versus active tumor targeting using RGDand NGR-modified polymeric nanomedicines," Nano Lett., vol. 14, no. 2, pp. 972981, 2014.

[67] W. Geldenhuys, T. Mbimba, T. Bui, K. Harrison, and V. Sutariya, "Brain-targeted delivery of paclitaxel using glutathione-coated nanoparticles for brain cancers," $J$. Drug Target., vol. 19, no. 9, pp. 837-845, Nov. 2011.

[68] S. H. Kim, J. H. Jeong, K. W. Chun, and T. G. Park, "Target-specific cellular uptake of PLGA nanoparticles coated with poly(L-lysine)-poly(ethylene glycol)folate conjugate.," Langmuir, vol. 21, no. 19, pp. 8852-7, 2005.

[69] W. Tai, R. Mahato, and K. Cheng, "The role of HER2 in cancer therapy and targeted drug delivery," J. Control. Release, vol. 146, no. 3, pp. 264-275, 2010.

[70] H. W. Yue, Q. C. Jing, P. P. Liu, J. Liu, W. J. Li, and J. Zhao, "Sphingosylphosphorylcholine in cancer progress," Int. J. Clin. Exp. Med., vol. 8, no. 8, pp. 11913-11921, 2015.

[71] S. Prabha and V. Labhasetwar, "Critical determinants in PLGA/PLA nanoparticlemediated gene expression.," Pharm. Res., vol. 21, no. 2, pp. 354-64, Feb. 2004.

[72] P. Kocbek, N. Obermajer, M. Cegnar, J. Kos, and J. Kristl, "Targeting cancer cells using PLGA nanoparticles surface modified with monoclonal antibody," $J$. Control. Release, vol. 120, no. 1-2, pp. 18-26, Jul. 2007. 
[73] P. Workman, E. O. Aboagye, F. Balkwill, A. Balmain, G. Bruder, D. J. Chaplin, J. A. Double, J. Everitt, D. A. H. Farningham, M. J. Glennie, L. R. Kelland, V. Robinson, I. J. Stratford, G. M. Tozer, S. Watson, S. R. Wedge, and S. A. Eccles, "Guidelines for the welfare and use of animals in cancer research.," Br. J. Cancer, vol. 102, no. 11, pp. 1555-77, 2010.

[74] M. Varna, P. Ratajczak, I. Ferreira, C. Leboeuf, G. Bousquet, and A. Janin, "In Vivo Distribution of Inorganic Nanoparticles in Preclinical Models," J. Biomater. Nanobiotechnol., vol. 3, no. May, pp. 269-279, 2012.

[75] C. W. Siah, J. Ombiga, L. A. Adams, D. Trinder, and J. K. Olynyk, "Normal iron metabolism and the pathophysiology of iron overload disorders.," Clin. Biochem. Rev., vol. 27, no. 1, pp. 5-16, 2006.

[76] R. P. Heaney and C. M. Weaver, Calcium and vitamin D, vol. 32, no. 1. 2003.

[77] NIH, “ImageJ Software,” https://imagej.nih.gov/ij/download.html, 2016.

[78] J. Liu, M. Yu, C. Zhou, and J. Zheng, "Renal clearable inorganic nanoparticles: A new frontier of bionanotechnology," Mater. Today, vol. 16, no. 12, pp. 477-486, 2013.

[79] E. Blanco, H. Shen, and M. Ferrari, "Principles of nanoparticle design for overcoming biological barriers to drug delivery," Nat. Biotechnol., vol. 33, no. 9, pp. 941-951, Sep. 2015.

[80] H. S. Choi, W. Liu, P. Misra, E. Tanaka, J. P. Zimmer, B. Itty Ipe, M. G. Bawendi, and J. V Frangioni, "Renal clearance of nanoparticles," Nat. Biotechnol., vol. 25, no. 10, pp. 1165-1170, 2007.

[81] P. . Michelle Longmire, Peter L. Choyke, M.D., and Hisataka Kobayashi, M.D., "Clearance Properties of Nano-sized Particles and Molecules as Imagin Agents: Consideration and Caveats," vol. 3, no. 5, pp. 703-717, 2012.

[82] W. M. Deen, M. J. Lazzara, and B. D. Myers, "Structural determinants of glomerular permeability.," Am. J. Physiol. Renal Physiol., vol. 281, no. 4, pp. F579-96, Oct. 2001.

[83] M. Ohlson, J. Sörensson, and B. Haraldsson, "A gel-membrane model of glomerular charge and size selectivity in series.," Am. J. Physiol. Renal Physiol., vol. 280, no. 3, pp. F396-405, Mar. 2001. 
[84] N. Heintz, L. Mamounas, and C. Gerfen, "The GENSAT Project," http://www.gensat.org/imagenavigator.jsp?imageID=19509\&showAtlas=false, 2016.

[85] S. M. Moghimi, A. C. Hunter, and J. C. Murray, "Long-circulating and targetspecific nanoparticles: theory to practice," Pharm. Rev, vol. 53, no. 2, pp. 283318, 2001.

[86] M. Bououdina, Handbook of Research on Nanoscience, Nanotechnology, and Advanced Materials. IGI Global, 2014.

[87] A. Omuro and L. DeAngelis, "Glioblastoma and Other Malignant Gliomas," JAMA, vol. 310, no. 17, pp. 1842-1850, Nov. 2013.

[88] K. Redmann, V. Müller, S. Tanneberger, and W. Kalkoff, "The membrane potential of primary ovarian tumor cells in vitro and its dependence on the cell cycle.," Acta Biol. Med. Ger., vol. 28, no. 5, pp. 853-6, 1972.

[89] G. Fullstone, J. Wood, M. Holcombe, and G. Battaglia, "Modelling the Transport of Nanoparticles under Blood Flow using an Agent-based Approach," Sci. Rep., vol. 5, p. 10649, 2015.

[90] R. Binggeli and I. L. Cameron, "Cellular potentials of normal and cancerous fibroblasts and hepatocytes," Cancer Res., vol. 40, no. 6, pp. 1830-1835, 1980.

[91] K. Cahill, "Molecular electroporation and the transduction of oligoarginines," Phys. Biol., vol. 7, no. 1, p. 16001, 2010.

[92] E.-K. Lim, Y.-M. Huh, J. Yang, K. Lee, J.-S. Suh, and S. Haam, "pH-triggered drug-releasing magnetic nanoparticles for cancer therapy guided by molecular imaging by MRI.," Adv. Mater., vol. 23, no. 21, pp. 2436-42, Jun. 2011.

[93] Y. Shen, H. Tang, M. Radosz, E. Van Kirk, and W. J. Murdoch, "pH-responsive nanoparticles for cancer drug delivery.," Methods Mol. Biol., vol. 437, pp. 183216, 2008.

[94] C. Wong, T. Stylianopoulos, J. Cui, J. Martin, V. P. Chauhan, W. Jiang, Z. Popović, R. K. Jain, M. G. Bawendi, and D. Fukumura, "Multistage nanoparticle delivery system for deep penetration into tumor tissue," Proc. Natl. Acad. Sci., vol. 108, no. 6, pp. 2426-2431, Feb. 2011.

[95] J. Kopeček, "Controlled biodegradability of polymers — a key to drug delivery systems," Biomaterials, vol. 5, no. 1, pp. 19-25, Jan. 1984. 
[96] G. Wu, A. Mikhailovsky, H. A. Khant, C. Fu, W. Chiu, and J. A. Zasadzinski, "Remotely Triggered Liposome Release by Near-Infrared Light Absorption via Hollow Gold Nanoshells," J. Am. Chem. Soc., vol. 130, no. 26, pp. 8175-8177, Jul. 2008.

[97] C.-Y. Lin, J.-R. Li, H.-C. Tseng, M.-F. Wu, and W.-L. Lin, "Enhancement of focused ultrasound with microbubbles on the treatments of anticancer nanodrug in mouse tumors," Nanomedicine Nanotechnology, Biol. Med., vol. 8, no. 6, pp. 900 907, Sep. 2016.

[98] A. K. Iyer, G. Khaled, J. Fang, and H. Maeda, "Exploiting the enhanced permeability and retention effect for tumor targeting," Drug Discov. Today, vol. 11, no. 17, pp. 812-818, 2006.

[99] G. Song, J. Petschauer, A. Madden, and W. Zamboni, "Nanoparticles and the mononuclear phagocyte system: pharmacokinetics and applications for inflammatory diseases," Curr. Rheumatol. Rev., vol. 10, no. 1, pp. 22-34, 2014.

[100] D. V Devine, K. Wong, K. Serrano, A. Chonn, and P. R. Cullis, "Liposomecomplement interactions in rat serum: implications for liposome survival studies.," Biochim. Biophys. Acta, vol. 1191, no. 1, pp. 43-51, Apr. 1994.

[101] S. N. Thomas, A. J. van der Vlies, C. P. O’Neil, S. T. Reddy, S. S. Yu, T. D. Giorgio, M. A. Swartz, and J. A. Hubbell, "Engineering complement activation on polypropylene sulfide vaccine nanoparticles.," Biomaterials, vol. 32, no. 8, pp. 2194-203, Mar. 2011.

[102] N. Doshi and S. Mitragotri, "Macrophages recognize size and shape of their targets.," PLoS One, vol. 5, no. 4, p. e10051, 2010.

[103] L. Babes, B. Denizot, G. Tanguy, J. J. Le Jeune, and P. Jallet, "Synthesis of Iron Oxide Nanoparticles Used as MRI Contrast Agents: A Parametric Study," J. Colloid Interface Sci., vol. 212, no. 2, pp. 474-482, Apr. 1999.

[104] M. D. Shultz, S. Calvin, P. P. Fatouros, S. A. Morrison, and E. E. Carpenter, "Enhanced ferrite nanoparticles as MRI contrast agents," J. Magn. Magn. Mater., vol. 311, no. 1, pp. 464-468, 2007.

[105] Y. Bao, T. Wen, A. C. S. Samia, A. Khandhar, and K. M. Krishnan, "Magnetic nanoparticles: material engineering and emerging applications in lithography and biomedicine," J. Mater. Sci., vol. 51, no. 1, pp. 513-553, 2016.

[106] S. Wohlfart, S. Gelperina, and J. Kreuter, "Transport of drugs across the bloodbrain barrier by nanoparticles.," J. Control. Release, vol. 161, no. 2, pp. 264-73, Jul. 2012. 
Alexandra Rodzinski

Born, Kremenchug, Ukraine

Education \& Awards

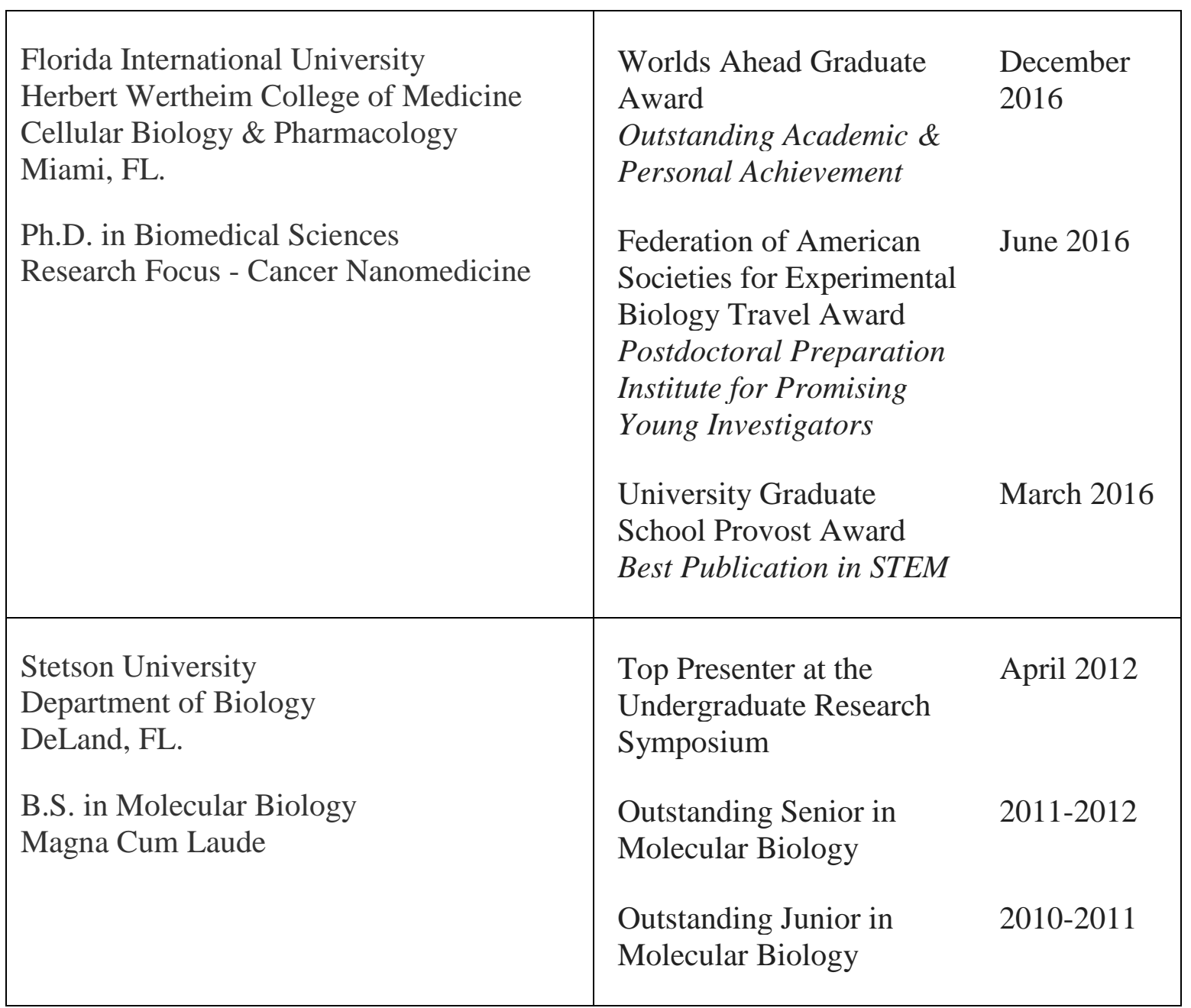

Publications \& Presentations

A. Rodzinski, C. Runowicz, and S. Khizroev. (2016) "Nanomedicine in cancer treatment." In preparation.

A. Hadjikhani, A. Rodzinski, C. Runowicz, and S. Khizroev. (2016) "Biodistribution and clearance of magnetoelectric nanoparticles in healthy and cancer-treated mice," In preparation. 
A. Nagesetti, A. Rodzinski, E. Stimphil, T. Stewart, C. Khanal, P. Wang, R. Guduru, P. Liang, I. Agoulnik, J. Horstmyer, and S. Khizroev. (2016) Multiferroic coreshell magnetoelectric nanoparticles as NMR sensitive nanoprobes for cancer cell detection. Currently in submission.

E. Stimphil, T. Stewart, P. Wang, A. Rodzinski, R. Guduru, B. Arkook, M.E. Itkis, P. Liang, C. Runowicz, and S. Khizroev. (2016) "Precision Nanomedicine: The Underlying Physics of Multiferroic Nanoparticles in a Cellular Microenvironment," Currently in submission.

A. Rodzinski, R. Guduru, P. Liang, A. Hadjikhani, T. Stewart, E. Stimphil, C. Runowicz, R. Cote, N. Altman, R. Datar, and S. Khizroev. (2016) "Targeted and controlled anticancer drug delivery and release with magnetoelectric nanoparticles," Scientific Reports 6, Article: 20867. doi:10.1038/srep20867

R. Guduru, P. Liang, J. Hong, A. Rodzinski, A. Hadjikhani, J. Horstmyer, E. Levister, and S. Khizroev. (2015) "Magneto-electric "spin" on stimulating the brain," Nanomedicine, Vol. 10, No. 13, Pages 2051-2061. doi:10.2217/nnm.15.52

A. Rodzinski, C. Runowicz, and S. Khizroev. "Targeted \& controlled anticancer drug delivery and release with magnetoelectric nanoparticles." Poster. Medical, Graduate, and Resident Research Symposium. April 29, 2016. Herbert Wertheim College of Medicine, Florida International University, Miami, FL.

A. Rodzinski, R. Guduru, P. Liang, A. Hadjikhani, T. Stewart, E. Stimphil, C. Runowicz, R. Cote, N. Altman, R. Datar, and S. Khizroev. "Targeted, controlled anticancer drug delivery and release with magnetoelectric nanoparticles." Poster Abstract Number 2204. American Association for Cancer Research (AACR) Annual Meeting. April 18, 2016. New Orleans, LA.

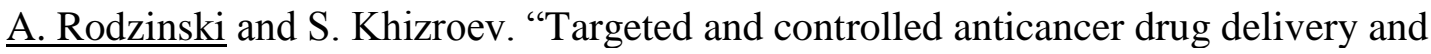
release with magnetoelectric nanoparticles." Seminar. Graduate Student Scholarly Forum. March 29, 2016. Florida International University, Miami, FL.

A. Rodzinski, R. Guduru, P. Liang, C. Runowicz, and S. Khizroev. "'Smart' Nanoparticles for Field-controlled Targeted Anticancer Drug Delivery and Release." Poster. March 4, 2015. Canesearch. Miller School of Medicine, University of Miami, Miami, FL.

A. Rodzinski and D. Stock. "The Mutagenic Effects of Cylindrospermopsin on Prokaryotic Organisms as Determined by the Ames Test." Seminar. April, 2012. Undergraduate Research Symposium. Stetson University, DeLand, FL. 